\author{
Universidade de São Paulo \\ Instituto de Física
}

\title{
Teorias com Grande Hierarquia de Escalas
}

\author{
Nayara Fonseca de Sá
}

Tese apresentada ao Instituto de Física para a obtenção do título de Doutor em Ciências

\section{Orientador:}

Prof. Dr. Gustavo Alberto Burdman (DFMA-IFUSP)

\section{Banca Examinadora:}

Prof. Dr. Gustavo Alberto Burdman (DFMA-IFUSP)

Prof. Dr. Oscar José Pinto Éboli (DFMA-IFUSP)

Prof. Dr. Rogério Rosenfeld (IFT-UNESP)

Prof. Dr. Eduardo Pontón Bayona (IFT-UNESP)

Prof. Dr. Gero Arthur Hubertus Thilo Freiherr von Gersdorff (PUC-RJ)

São Paulo

2016 


\section{FICHA CATALOGRÁFICA}

Preparada pelo Serviço de Biblioteca e Informação do Instituto de Física da Universidade de São Paulo

Sá, Nayara Fonseca de

Teorias com grande hierarquia de escalas : Theories with large hierarchy of scale. São Paulo, 2016.

Tese (Doutorado) - Universidade de São Paulo. Instituto de Física. Depto. de Física Matemática.

Orientador: Prof. Dr. Gustavo Alberto Burdman

Área de Concentração: Física

Unitermos: 1. Física de partículas; 2. Física de alta energia; 3. Física teórica. 


\author{
University of São Paulo \\ Physics Institute
}

\title{
Theories with Large Hierarchy of Scales
}

\author{
Nayara Fonseca de Sá
}

Thesis presented to the
Physics Institute to obtain
the title of Doctor in Sciences

\section{Advisor:}

Prof. Dr. Gustavo Alberto Burdman (DFMA-IFUSP)

\section{Thesis Committee:}

Prof. Dr. Gustavo Alberto Burdman (DFMA-IFUSP)

Prof. Dr. Oscar José Pinto Éboli (DFMA-IFUSP)

Prof. Dr. Rogério Rosenfeld (IFT-UNESP)

Prof. Dr. Eduardo Pontón Bayona (IFT-UNESP)

Prof. Dr. Gero Arthur Hubertus Thilo Freiherr von Gersdorff (PUC-RJ)

São Paulo

2016 

À minha mãe, Elza Fonseca. 



\section{Acknowledgments}

Sou muito grata ao meu orientador, Gustavo Burdman, por toda confiança depositada, pelo tempo investido, pelo aprendizado durante todos esses anos e pela liberdade que me proporcionou descobrir as questões que me intrigam. Tive a imensa sorte de ter um orientador por quem tenho grande admiração e que influenciou de forma decisiva na maneira como vejo a ciência.

Quero agradecer à minha família por compreender minha ausência em diversos momentos importantes. Ao meu noivo, Igor Carboni, pelo apoio incondicional, por dividir os prós e contras da vida acadêmica e por ser uma fonte de inspiração. À minha mãe, Elza Fonseca, por me apoiar em todas as escolhas da minha vida e de quem eu ouvi pela primeira vez 'você tem fazer o que você gosta'. Me faltam palavras para expressar a importância de vocês em minha vida.

I thank the members of my thesis committee, Gustavo Burdman, Oscar Éboli, Gero Gersdorff, Eduardo Pontón, and Rogério Rosenfeld for their time and valuable comments.

I want to thank Nicolás Bernal, Stefano Finazzo, Chee Sheng Fong, Leonardo de Lima, Camila Machado, and Zahra Tabrizi for proofreading the thesis.

While in graduate school I had the opportunity to work with researchers and students from different institutions. I wish to thank Nicolás Bernal, Gustavo Burdman, Chee Sheng Fong, Renata Funchal, André Lessa, Gabriela Lichtenstein, Leonardo de Lima, Laura Lopez-Honorez, Camila Machado, Ricardo Matheus, Lina Necib, and Jesse Thaler for everything I have learned from them during these collaborations.

I also thank Jesse Thaler for hosting me at the Center for Theoretical Physics (MIT), where I was lucky to overlap with Gilly Elor, Lina Necib, Nick Rodd, and Tracy Slatyer. I thank you all for the great time at MIT. Many thanks to Tracy Slatyer for introducing me to research in dark matter and for the enjoyable Harvard-MIT dark matter lunch meetings.

I want to thank Géraldine Servant for hosting me at the DESY Theory group in Hamburg, where I had an amazing time. I thank Iason Baldes, Sebastian Bruggisser, 
Christophe Grojean, Thomas Konstandin, Janis Kummer, Laura Sagunski, Kai SchmidtHoberg, Pedro Schwaller, and Géraldine Servant for the great physics and delicious cakes.

I thank the hospitality of the members of the Department of Computer Science and Butler Library at Columbia University, Rotch Library (MIT), and Instituto de Física Teórica (UNESP), where I spent a reasonable amount of time during the development of this thesis. In particular, I want to thank the Instituto de Física Teórica for the unlimited supply of coffee.

Agradeço ao meu orietador de iniciação científica, Alberto Saa, pelos conselhos valiosos. Lembro-me de quando conversávamos sobre minha transição da Unicamp para a USP, e ele me disse que eu saberia reconhecer quando estivesse 'vivendo a pós-graduação' no IF-USP. Eu descrevo tal experiência como a combinação de aprendizado e questionamentos que se conciliados com amigos com quem se pode discutir sem qualquer pudor ou hierarquia, resulta em algo inquietante e transformador. Devo essa vivência aos meus contemporâneos: Léo de Lima, Camila Machado, Hugo Marrochio e Yuber Pérez, com quem tive a sorte de conviver durante alguns anos no IF-USP. Vocês têm meu respeito e admiração. Obrigada pelos cafés, ótima física e memórias que não se apagarão.

I would like to thank many other friends and colleagues from USP and Unesp, which made my days in São Paulo less lonely; my special thanks to Antonio, Denise, Dudu, Eliane, Gabi, Jaque, Jorgivan, Karine, Maria, Nico, Nubia, Pedro, Ricardo, Sheng, Stefano, Tiago, and Zahra.

Quero agradecer também aos amigos de longa data da minha cidade natal, Volta Redonda, e de Campinas, Juliana Barbosa, Carlitcha Matsumoto, Izis de Melo, Douglas Mendes, Carol Pimentel, Zé Renato, Larissa Sayuri, Marina Trad e Allan Vilela.

Também gostaria de agradecer aos funcionários do Departamento de Física Matemática e da secretaria de pós-graduação do Instituto de Física por toda assistência prestada durante esse período.

Finalmente, agradeço ao CNPq e à FAPESP (processo 2011/10047-9) pelo apoio financeiro. 


\section{Abstract}

In this thesis we explore a class of $N$-site models that were developed to generate large-scale hierarchies. Using the dimensional deconstruction approach and appropriate matchings, these purely four-dimensional theories coincide with $\mathrm{AdS}_{5}$ models in the continuum limit, which corresponds to the limit with a large number of gauge groups. On the other hand, in the coarse lattice limit such theories have very distinct couplings of the excited states to zero mode fields compared with $\mathrm{AdS}_{5}$ constructions, resulting in a rich phenomenology to be explored at the Large Hadron Collider (LHC). The Standard Model (SM) hierarchy problem is solved if the Higgs field is infrared-localized as in Randall-Sundrum scenarios. The SM fermion mass hierarchy and mixings are obtained by different localizations of zero mode fermions in the theory space.

This framework is employed to tackle the electroweak hierarchy problem from a new perspective. We show that an effective few site description of a warped extra dimension can implement the recently introduced relaxion models, which are a new alternative to explain the radiative stability of the SM scalar sector through the cosmological relaxation mechanism. These models require very large field excursions, which are difficult to generate in a consistent ultraviolet completion and to reconcile with the compact field space of the relaxion. We propose an $N$-site model that naturally generates the large decay constant needed to address these problems. In our model, the mass matrix of the pseudo-Nambu-Goldstone Bosons (pNGBs), whose zero mode plays the role of the relaxion field, is identical to the one obtained for a pNGB Wilson line in the deconstruction of $\mathrm{AdS}_{5}$.

Keywords: $N$-site models, large-scale hierarchies, dimensional deconstruction, warped extra dimension, cosmological relaxation mechanism, pseudo-Nambu-Goldstone boson. 


\section{Resumo}

Nesta tese exploramos uma classe de modelos de $\mathrm{N}$-sítios que foram desenvolvidos para produzir grandes hierarquias de escalas. Usando a abordagem de desconstrução dimensional e correspondências apropriadas, esses modelos puramente quadridimensionais coincidem com modelos $\mathrm{AdS}_{5}$ no limite do contínuo, que corresponde ao limite com um grande número de grupos de gauge. Por outro lado, no limite em que há poucos grupos de gauge, tais teorias possuem os acoplamentos entre estados excitados e modos zero dos campos muito distintos dos acoplamentos em construções $\mathrm{AdS}_{5}$, resultando em uma rica fenomenologia a ser explorada no Large Hadron Collider (LHC). O problema da hierarquia do Modelo Padrão (MP) é resolvido se o campo de Higgs está localizado no infravermelho, assim como nas teorias Randall-Sundrum. A hierarquia de massa dos férmions do MP e misturas são obtidas pelas diferentes localizações dos modos zero dos férmions no espaço dos sítios.

Essa estrutura é empregada para abordar o problema da hierarquia sob uma nova perspectiva. Nós mostramos que uma descrição efetiva com poucos sítios de uma dimensão extra curva pode implementar os recentemente introduzidos modelos de relaxion, que são uma nova alternativa para explicar a estabilidade radiativa do setor escalar do MP através do mecanismo de relaxação cosmológico. Esses modelos requerem que o campo experimente grandes variações, que são difíceis de serem geradas em um modelo ultravioleta consistente e de serem compatíveis com o espaço compacto do relaxion. Nós propomos um modelo de $\mathrm{N}$-sítios que gera naturalmente essa grande constante de decaimento necessária para abordar esses problemas. No nosso modelo, a matriz de massa dos pseudo Bósons de Nambu-Goldstone (pBNGs), cujo modo zero faz o papel do relaxion, é idêntica à matriz obtida para uma linha de Wilson pBNG na desconstrução de $\mathrm{AdS}_{5}$.

Palavras-chave: modelos de $N$-sítios, grande hierarquia de escalas, desconstrução dimensional, dimensão extra curva, mecânismo de relaxação cosmológico, pseudo bóson de Nambu-Goldstone. 


\section{Bibliographic Note}

The following works were completed during the development of this thesis:

1. N. Bernal, C. S. Fong and N. Fonseca, Sharing but not Caring: Dark Matter and the Baryon Asymmetry of the Universe, JCAP 1609, 005 (2016).

2. N. Fonseca, L. de Lima, C. S. Machado and R. D. Matheus, Large field excursions from a few site relaxion model, Phys. Rev. D 94, 015010 (2016).

3. N. Fonseca, L. Necib and J. Thaler, Dark Matter, Shared Asymmetries, and Galactic Gamma Ray Signals, JCAP 1602, 052 (2016).

4. N. Fonseca, R. Z. Funchal, A. Lessa and L. Lopez-Honorez, Dark Matter Constraints on Composite Higgs Models, JHEP 1506, 154 (2015).

5. G. Burdman, N. Fonseca and G. Lichtenstein, Resonances from Quiver Theories at the LHC, Phys. Rev. D 88, 116006 (2013).

6. G. Burdman, N. Fonseca and L. de Lima, Full-hierarchy Quiver Theories of Electroweak Symmetry Breaking and Fermion Masses, JHEP 1301, 094 (2013).

References $[2,5,6]$ listed above compose the main content of this thesis, while papers $[1,3,4]$ cover results related to non-minimal dark matter scenarios that are summarized in Chapter 1 and are not further discussed in this thesis. 


\section{Contents}

Acknowledgments $\quad$ v

Abstract vii

Resumo ix

Bibliographic Note xi

1 Introduction $\quad 1$

1.1 The Electroweak Hierarchy Problem . . . . . . . . . . . . . . 5

1.2 Going Beyond . . . . . . . . . . . . . . . . . . 9

1.2.1 How to Generate Large-Scale Hierarchies? . . . . . . . . . . . 9

1.2.2 From Particle Physics to Cosmology and Back . . . . . . . . . 10

1.3 Structure and Proposal of this Thesis . . . . . . . . . . . . . . . . . 11

2 Introduction to Quiver Theories $\quad 13$

$2.1 \quad \mathrm{~N}$-site Models and Large-Scale Hierarchies . . . . . . . . . . . . . . . 13

2.2 Matching with a Deconstructed Extra-Dimension . . . . . . . . . . . 19

2.3 A Remnant pseudo-Nambu-Goldstone Boson . . . . . . . . . . . . . . 21

3 Localization in the Theory Space $\quad 25$

3.1 Including the Fermions . . . . . . . . . . . . . . . . . . 25

3.2 Gauge Couplings . . . . . . . . . . . . . . . . . . . 30

3.3 Model with a Few Sites . . . . . . . . . . . . . . . . 33

3.3.1 Flavor Violation Bounds . . . . . . . . . . . . . . 37

3.3.2 Electroweak Precision Parameters . . . . . . . . . . . . . 41 
3.4 Resonances from Quiver Theories . . . . . . . . . . . . . . . . . . . 46

4 Realizing the Relaxion with $N$-site Models 51

4.1 The Relaxion Idea . . . . . . . . . . . . . . . . . . . . 51

4.2 Minimal Model . . . . . . . . . . . . . . . . 53

4.3 Higgs-Axion Interplay . . . . . . . . . . . . . . . . . . . 57

$\begin{array}{lll}5 & \text { Conclusions and Remarks } & 61\end{array}$

$\begin{array}{ll}\text { Bibliography } & 64\end{array}$ 


\section{Chapter 1}

\section{Introduction}

Particle physics has always been crucial for the understanding of the evolution of the universe. On the other hand, one should ask what the cosmological history can teach us about our models of fundamental particle interactions. Indeed, cosmology can be a key ingredient to answer some open questions in elementary particle physics and the promising bridge between these two areas may show us unexpected directions. ${ }^{1}$ In the past twenty years, cosmology has entered its 'precision era' and its interplay with particle physics became more appealing. In fact, the data obtained from large-scale structure surveys and by mapping of the Cosmic Microwave Background (CMB) radiation have enabled us to improve our knowledge of cosmological parameters from about within an order of magnitude to the percent level. After COBE and WMAP, the ESA satellite Planck has significantly improved the precision cosmological measurements ensuring more detailed maps of CMB anisotropies [2,3]. Although the standard model of cosmology (or $\Lambda$ CDM) is in agreement with the current data, there are some anomalies that are far from being fully understood (see e.g. Ref. [4]).

On the other hand, the Standard Model (SM) of elementary particles has been proven extremely successful in describing the current experimental data [5]. It predicts a remnant state from the electroweak symmetry breaking, the Higgs field, which is consistent with the observed boson at the Large Hadron Collider (LHC) in

\footnotetext{
${ }^{1}$ It should be mentioned that this year the Laser Interferometer Gravitational-Wave Observatory (LIGO) announced the first direct observation of gravitational waves [1], which opens a new era of gravitational astronomy that might reveal surprising features about the fundamental physics.
} 
2012 [6,7]. If this Higgs-like particle is in fact the SM Higgs ${ }^{2}$, it remains unanswered what protects the Higgs mass from receiving corrections of the ultraviolet (UV) cutoff of the theory. From this issue results the so-called gauge hierarchy problem [10,11], whose natural solution indicates new physics close to the TeV scale $[12,13]$ as it is explained in the Sec. 1.1.

Together with the electroweak hierarchy problem, there are several open questions in particle physics and also in cosmology [14-16]. In the following, we are going to mention some of them. A common feature among these problems is that there is no compelling indication of at which scale the new physics might appear and we do not know if such an energy can be achieved in the current and next generation of experiments. This is a key difference between all these other unsolved questions and the SM hierarchy problem. As a noticeable example, we can point out the fact the SM does not provide any explanation for the large hierarchy of fermion masses and CKM matrix elements. These values are simply chosen in order to match the experimental data [5]. This issue is also explored in this thesis in Chap. 3, where we obtained the fermion mass and CKM hierarchies through the different localizations of zero mode fermions in the theory space, which due to the warping localization factor, can be achieved by adjusting $\mathcal{O}(1)$ parameters.

There is also an important evidence pointing to physics beyond SM, which is the fact that due to the oscillation phenomenon we know that the neutrinos have mass (see e.g. [17, 18]). In 2015, the experimental discovery of neutrino oscillation by the Super-Kamiokande Observatory and the Sudbury Neutrino Observatory was recognized with the Nobel Prize [19]. This is another indication of an intermediate scale between the electroweak and the Planck mass, which reminds us about the SM hierarchy problem. ${ }^{3}$

Another problem is related to the fact that the QCD Lagrangian admits a term that violates the $\mathrm{CP}$ symmetry, which results in the so called strong $\mathrm{CP}$ problem. As this effect is not observed, one can obtain an upper bound on the coefficient of this term which comes from neutron electric dipole moment experiments and it is about $|\theta| \lesssim 10^{-10}$ [23], leading to another fine-tuning in the SM. A possible solution

\footnotetext{
${ }^{2}$ For the latest LHC Higgs measurements see Refs. [8,9].

${ }^{3}$ Actually there are proposals that claim to explain the neutrino masses and to avoid the naturalness problem if the right-handed neutrinos are lighter than $\sim 10^{7} \mathrm{GeV}[20-22]$.
} 
to this problem was proposed by Peccei and Quinn $[24,25]$ through the introduction of a new particle, the axion field, which can dynamically make that coefficient zero. The QCD axion has not been observed yet and it is subject to many constraints from direct detection experiments, astrophysics and cosmology [5, 26].

The previous discussion shows that there are many hints for new physics already in the structure of the SM itself. In addition, the SM clearly does not describe astrophysical and cosmological data. A compelling example is the fact it cannot explain the matter-antimatter asymmetry observed in the universe, which can be quantified by the ratio $\eta=\left(n_{b}-n_{\bar{b}}\right) / n_{\gamma} \simeq 10^{-10}[27]$, where $n_{b}, n_{\bar{b}}$, and $n_{\gamma}$ refer to the number densities of baryons, anti-baryons, and photons, respectively. Furthermore, the SM has no candidate to the non-baryonic matter that we called as dark matter (DM), which is necessary to explain a large amount of data such as the cosmic microwave background fluctuation spectrum, the structure formation, and the galaxies rotation curves [27-29]. The weakly interacting massive particle (WIMP) paradigm is a compelling possibility if the DM is composed of a single new particle (see [30] for a review). However, as the SM has a vast and subtle structure, it is reasonable to explore a comparable rich dynamics in the dark sector. For instance, this new sector may share some of the properties of the visible matter, such as composite states, accidental symmetries, and asymmetric abundances. Keeping this in mind and taking the advantage of the fact that many of the theoretical DM candidates can now be tested by different experimental strategies such as astro-cosmo tests, direct, indirect and collider searches [31,32], we explored non-minimal DM models and asymmetric DM scenarios, as it is discussed in the following.

Composite Dark Matter [33]: In the context of non-minimal dark matter scenarios, we explored in [33] the dark matter constraints on composite Higgs models. In these theories, the Higgs doublet is a pseudo-Nambu-Goldstone boson appearing in the low energy theory as a result of the spontaneous breaking of a global symmetry $G \rightarrow H$ by new strong sector dynamics.

Based on this framework, we discuss in [33] how the DM observables (relic abundance, direct and indirect detection) constrain the dimension- 6 operators induced by the strong sector, assuming that DM behaves as a WIMP and that the relic abundance is set by the freeze-out mechanism. We apply our general results to 
$S O(6) / S O(5)$ and $S O(6) / S O(4) \times S O(2)$, which contain a singlet and doublet DM candidate, respectively. In particular, we find that if compositeness is a solution to the little hierarchy problem, DM representations larger than the triplet are strongly disfavored.

Asymmetric Dark Matter and Indirect Detection Signals [34]: asymmetric dark matter (ADM) models are motivated by the hypothesis that the present-day dark matter abundance originated from an asymmetry in the number density of particles and antiparticles in the early universe. If the DM and baryon asymmetries had a common origin, this would explain the closeness of the DM and the baryon abundances. ${ }^{4}$

Typical ADM models face the challenge of lacking potential indirect detection signatures. Our work [34] considers semi-annihilation process through which it is possible to produce dark matter indirect signals in the asymmetric paradigm. In this scenario, the asymmetry sharing is efficient until temperatures below the DM mass due to light unstable states carrying baryon number. Consequently, the resulting DM abundance is set by the thermal freeze-out of the semi-annihilation process instead of by the decoupling of high-scale interactions. In this framework, the processes in charge of asymmetry sharing in the early universe can produce signals today in indirect detection experiments. Interestingly, there is a region of parameter space compatible with the observed Fermi excess of GeV gamma rays from the galactic center $[36,37]$.

Dark Matter and the Baryon Asymmetry of the Universe [38]: In this work, we discuss scenarios where dark matter particles carry baryon and/or lepton numbers. As a consequence, the DM fields become intimately linked to the Standard Model ones and can be maximally asymmetric just like the ordinary matter. We consider an initial asymmetry frozen in either the SM or the DM sector; the main role of the transfer operators is to properly share the asymmetry between the two sectors, in accordance with observations. After the chemical decoupling, the DM

\footnotetext{
${ }^{4}$ Using the measurements of the primordial deuterium abundance of the big bang nucleosynthesis [35] and the temperature anisotropies in the cosmic microwave background (CMB) [27], the abundance of baryons can be independently determined to be about $\Omega_{B} h^{2} \simeq 0.02$. Additionally, the CMB measurement also determines the amount of DM to be $\Omega_{D M} h^{2} \simeq 0.12$ [27].
} 
and SM sectors barely talk to each other as there is only an ineffective interaction between them. Once the DM mass is specified, the Wilson coefficients of these operators are fixed by the requirement of the correct transfer of the asymmetry. We study the phenomenology of this framework at colliders, direct detection and indirect detection experiments. The LHC phenomenology is very rich and can be tested in different channels such as monojet with missing energy and monojet plus monolepton and missing energy.

\subsection{The Electroweak Hierarchy Problem}

\section{A First Look: a condensed matter analogy}

Let us consider a condensed matter system where the low-energy description breaks down at the cutoff scale $\Lambda$, which represents the inverse of the atomic spacing a, i.e. $\Lambda=1 / a$. As a concrete example, we will describe the ferromagnet [39, 40], which loses its magnetization when it is heated at temperatures higher than some critical temperature $\left(T_{C}\right)$. This is a well-known example of a system with order-disorder phase transition from which we can gain some intuition about the electroweak hierarchy problem. ${ }^{5}$ This phase transition can be parametrized by an order parameter $\phi(x)$. For instance, $\phi$ can describe the local spin density projected in some axis, representing the strength of magnetization in the ferromagnet. At temperatures near the $T_{C}$, the phase transition of this system is well described by the Ginzburg-Landau effective Lagrangian, as follows:

$$
\mathcal{L}=b\left(T-T_{C}\right) \phi^{2}+c \phi^{4}+\cdots
$$

where for these temperatures $b$ and $c$ can be treated as real and positive numbers. The effective mass-squared for the scalar is $m^{2}=2 b\left(T-T_{C}\right)$, then $m^{2}$ is positive for $T>T_{C}$ and negative for temperatures below the critical one, indicating that a spontaneous symmetry breaking has taken place. Intuitively, the effective mass defines the coherence length of the system, i.e. $\xi \sim 1 / m$, which is analogous to

\footnotetext{
${ }^{5}$ A typical way to introduce the SM hierarchy problem is through the analogy between quantum field theory and statistical mechanics [39,40], which we learned from Kenneth G. Wilson and others in the early 1970 s through the renormalization group (see e.g. [41-43]).
} 
Compton wavelength in the Yukawa potential. From (1.1) we see that only at temperatures very close to the critical one $\left(T \simeq T_{C}\right)$, it is possible to have $m \ll \Lambda$; and indeed, the correlation would be of long-range type in this case.

While it is reasonable to imagine that one can delicately choose a specific temperature in a condensed matter system through the experimental apparatus, this is not the case when one considers the fine-tuning of the Higgs mass in the SM. Of course, if the SM were valid up to arbitrary high energies, there would be no hierarchy problem, since in this case there is no physical cutoff. However, as a quantum field theory, the SM has its domain of validity and its upper bound is at most at energies close to the Planck mass $\left(M_{\mathrm{Pl}} \sim 10^{19} \mathrm{GeV}\right)$, where we expect that the gravitational effects are important. At these high energies the SM must be completed to a more fundamental theory, i.e. a theory of quantum gravity.

\section{A Closer Look: scalar masses are UV sensitive}

According to 't Hooft [11], a parameter is said 'technically natural' if the theory has an enhanced symmetry once this parameter is set to zero. This can also be seen from the fact that the quantum corrections to such a parameter are proportional to the parameter itself, i.e. the parameter is stable under quantum corrections. Let us consider now our case of study: the scalar field. Schematically, we can write the running mass of a scalar of mass $m_{S}$ that couples to another state of mass $M$ as

$$
\frac{d m_{S}^{2}}{d \log \mu}=\beta_{1} M^{2}+\beta_{2} m_{S}^{2}
$$

where $\mu$ is the renormalization scale and $\beta_{1}, \beta_{2}$ are coefficients that depend on $\mu$. Note that the term proportional to $\beta_{2}$ does not introduce large corrections since it depends on the mass parameter itself. In quantum field theories, there is a crucial difference between the running mass of scalars and fermionic or vector fields, which is the fact that the mass of a fundamental scalar field is not protected by any symmetry, i.e. it can receive corrections proportional to the mass of any state that couples to it (as the term proportional to $\beta_{1}$ in (1.2)). On the other hand, this is not the case for fermion and vector fields in the SM, since the corrections to their mass parameters are proportional to the masses themselves, which is in agreement to the 
fact that they are respectively protected by the chiral and gauge symmetries $[39,40]$. In the SM, the quantum corrections to the Higgs mass are unstable as there is no symmetry to protect it. In particular, it is UV sensitive as the Higgs may couple to heavy states with masses close to the cutoff of the theory, which can be seen explicitly from (1.2) with $M \gg m_{S}$. To make this discussion clearer, let us consider again the scalar field $\phi$ of mass $m_{S}$ that couples to a heavy Dirac fermion $\psi$ of mass $M$ through the Yukawa interaction $\mathcal{L}=\lambda \phi \bar{\psi} \psi$, where $\lambda$ is the Yukawa coupling. The one-loop contribution to the scalar mass due to the interaction with the fermion is represented in Fig. 1.1. One can use dimensional regularization and $\overline{\mathrm{MS}}$ subtraction

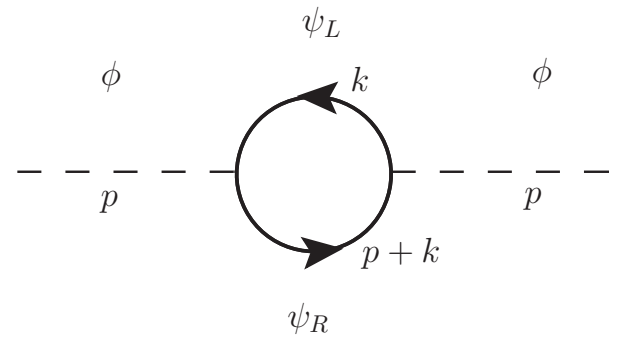

Figure 1.1: Contribution at 1-loop to the mass of the scalar $\phi$ due to the interaction with the fermion $\psi \cdot p$ refers to the external momentum and $k$ is the momentum in the loop.

scheme to compute the renormalized mass $\left.\left(\delta m^{2}\right)_{S, \text { full }}^{\overline{\mathrm{MS}}, 1-\text { loop }}\right|_{\mu=M} \sim-\lambda^{2} M^{2}+\cdots$, where the dots refer to terms that do not depend quadratically on $M[39,40,44,45]$. Therefore, assuming that $M$ is much larger than the $\phi$ mass measured value (i.e. $M \gg m_{S \text {,eff }}^{2}$ ), in order to match the effective mass-squared, one needs to delicately cancel out the 1-loop contribution (proportional to $M^{2}$ ) with the bare mass term. More specifically, assuming that $\lambda \sim \mathcal{O}(1)$ and that the scalar mass measured value is $m_{S \text {,eff }}^{2}=(125 \mathrm{GeV})^{2}$; for a heavy fermion of mass near the GUT scale $M^{2}=$ $\left(10^{15} \mathrm{GeV}\right)^{2}$, one gets $m_{S \text {,eff }}^{2} / \delta m_{S}^{2} \approx 10^{-26}$, meaning that it is necessary a cancellation of about one part in $10^{26}$ between two a priori independent parameters in order to get the scalar mass at the electroweak scale. It is important to mention that $M$ is not present here due to a mathematical artifact that we used to regularize the theory; instead, $M$ is a physical quantity, i.e. the mass of the heavy fermion that couples to the scalar field. This discussion summarizes the real nature of the hierarchy 
problem: the quadratic sensitivity to the UV degrees of freedom; or in other words, the large dependence of measurements at low energy to tiny deformations of a theory defined at high energy scales. Therefore, if the boson observed by the ATLAS [6] and CMS [7] in 2012 is indeed the SM Higgs, it is definitely established the question of what protects the Higgs mass from receiving corrections of the UV cutoff of the theory. From this perspective, it is natural to expect that new physics might exist at $\mathcal{O}(1) \mathrm{TeV}$ such that no large fine-tuning is necessary to adjust the Higgs mass.

On the other hand, one can speculate that there is nothing beyond the SM between the electroweak and the Planck scale, which is a strong assumption as it requires a correct description of nature by a single model in a huge energy range. Since at energies near the Planck mass the quantum effective field theory description should not be trusted anymore, one can argue that the hierarchy problem described from this perspective (as discussed before) is nonsense. Although there is nothing wrong with this assumption, this is very unlikely as physics beyond the SM is necessary to explain other open issues in particle physics and cosmology (for example to explain dark matter and baryon asymmetry of the universe, as we discussed previously in this chapter). Then, once one supposes that the SM is valid up to the Planck scale, this necessarily leads to the assumption that all new physics needed to solve other open questions is going to appear only at this scale.

Additionally, there is an upper bound on the Higgs mass due to the unitarity of the electroweak theory [46]. The requirement that the perturbative approach has to be meaningful sets a maximum value to the Higgs mass. For instance, one can get such a limit by computing the energy necessary to unitarize scattering channels whose amplitudes depend on the Higgs mass; through the scattering of longitudinal gauge bosons as $W_{L}^{+} W_{L}^{-} \rightarrow W_{L}^{+} W_{L}^{-}$or $W_{L}^{+} W_{L}^{-} \rightarrow Z_{L} Z_{L}$. In the SM, the Higgs mass cannot be larger than $710 \mathrm{GeV}$ [13] in order to respect the unitarity bound. There is also the issue regarding the Higgs potential metastability $[47,48]$. Assuming the SM only, i.e. that the boson observed at the LHC is the SM Higgs, one can conclude that the SM scalar potential gets unstable at about $10^{11} \mathrm{GeV}$; fortunately, the lifetime of the electroweak vacuum is much longer than the age of the universe [49]. 


\subsection{Going Beyond}

\subsubsection{How to Generate Large-Scale Hierarchies?}

The electroweak hierarchy problem can be naturally solved in warped extra dimension constructions as the Randall-Sundrum (RS) model [50,51], which is a 5D theory with an extra dimension in an anti-de Sitter (AdS) background. Due to the warp factor, a displacement in the extra dimension corresponds to an exponential factor in the ordinary coordinates. To see this explicitly, one can consider the $\mathrm{AdS}_{5}$ metric,

$$
d s^{2}=e^{-2 k|y|} \eta_{\mu \nu} d x^{\mu} d x^{\nu}-d y^{2},
$$

where $y$ is the coordinate in the extra dimension, $k$ is the AdS curvature, and $\eta_{\mu \nu}$ is the Minkowski metric in four dimensions. Notice that this metric is invariant under the transformations: $y \rightarrow y+d, x \rightarrow e^{k d|y|} x$; so that a displacement in $y$ results in an exponentially suppressed factor in the energy scale $\partial_{\mu} \rightarrow e^{-k d|y|} \partial_{\mu}$. The usual assumption in $\mathrm{AdS}_{5}$ models is that they are dual to $4 \mathrm{D}$ strongly coupled theories (see e.g. [52-55] for reviews). The minimal formulation of RS models has severe problems with flavor violation at tree level [56-58]. For instance, to overcome the kaon physics constraints without introducing new global flavor symmetries, these models require that the mass scale of the first excited state is larger than a few tens of $\mathrm{TeV}$ [59-61], which generates an undesirable hierarchy between the first Kaluza-Klein state and the electroweak scale.

In this thesis, we study the quiver (or moose) theories $[62,63]$ which are a class of four-dimensional models that share many features with warped dimensional constructions. In Ref. [64], we present a new class of theories built to generate largescale hierarchies, which we denominate full-hierarchy quiver theories. Using the dimensional deconstruction approach and appropriate matchings, these purely fourdimensional theories coincide with $\mathrm{AdS}_{5}$ models in the continuum limit [65], which corresponds to the limit with a large number of gauge groups.

On the other hand, full-hierarchy quiver theories in the coarse lattice limit have very distinct couplings of excited gauge bosons to zero mode fermions compared with $\mathrm{AdS}_{5}$ models, resulting in a rich phenomenology to be explored. The hierarchy problem is solved if the Higgs field is infrared-localized as in the warped extra dimen- 
sion models. We also obtained the fermion mass hierarchy by different localizations of zero mode fermions in the theory space. This procedure is similar to the localizations of zero mode fermions in the extra dimension in $\mathrm{AdS}_{5}$ theories, but without resulting in large flavor violation which severely constrains the minimal formulation of RS models [66,67]. We study the electroweak precision bounds on quiver models with few gauge groups, and show that we do not need to impose an extension in the electroweak gauge sector in order to avoid large contributions to the $T$ parameter at tree level. Further, in Ref. [68], we derive bounds from the LHC data on the color-singlet and color-octet excited gauge bosons from their decays to jets and top pairs.

\subsubsection{From Particle Physics to Cosmology and Back}

Many SM extensions that were proposed to solve the hierarchy problem are now being severely constrained by the LHC data. The quadratic sensitivity of the Higgs mass to UV degrees of freedom has been one of the leading motivations for searches of new physics at the LHC, whose most popular attempts are supersymmetry [69] and compositeness [70]. The speculation era regarding this matter is approaching an end through the Run II that started in 2015. Before the Run I, which started in 2011, the expectation for a significant amount of the community was to find new physics close to the TeV scale during the first years of LHC operation. Now this scenario has been changing since there is no definitive evidence of physics beyond the SM in the current data. From the experimental side, one cannot categorically say that the new data will answer urgent and well-debated questions; but surely, it will provide us a better understanding of the fundamental physics. From the theoretical point-ofview, it is an opportune moment to explore unusual possibilities and rethink baroque statements, for instance by searching for connections to surrounding research areas. On the interchange between theoretical and experimental fronts, it is interesting to develop complementary searches to connect the LHC physics and other experimental strategies. $^{6}$

Following this direction by taking the advantage of the interplay between particle

\footnotetext{
${ }^{6}$ See Refs. [71,72] where it is proposed atomic probes of physics beyond the Standard Model and Refs. [73-75] for discussions regarding complementarity of dark matter searches.
} 
physics and cosmology, the relaxion models $[76,77]$ use the cosmological evolution to explain the smallness of the Higgs mass. These models are a new alternative to explain the radiative stability of the SM scalar sector through the cosmological relaxation mechanism of the electroweak scale. The idea was inspired by Abbott's attempt [78] to solve the cosmological constant problem ${ }^{7}$, which did not work since his mechanism besides cancelling the cosmological constant with high precision, it also removes all the matter as well, resulting in an 'empty universe' (see also [83-85] for similar ideas).

On the other hand, the relaxion models require very large field excursions, which are difficult to generate in a consistent UV completion and to reconcile with the compact field space of the relaxion. In Ref. [86] we propose an $N$-site model which naturally generates the large decay constant needed to address these issues. Interestingly, our model admits a continuum limit when the number of sites is large, which may be interpreted as a warped extra dimension.

\subsection{Structure and Proposal of this Thesis}

In this thesis we focus on the development of theories that can generate largescale hierarchies. We were inspired by different model building frameworks such as dimensional deconstruction techniques, composite Higgs models, and warped extra dimension scenarios. The theoretical tools studied here can be applied to particle physics and cosmology; and in fact, from the interchange of these two fields new perspectives may appear. In the following, we briefly describe the contents of each chapter.

Chapter 2: Introduction to Quiver Theories. In this chapter, we show the general framework and model building for $N$-site models, focusing on constructions that can generate large hierarchy of scales. The matching of these theories with a deconstructed warped extra dimension is also discussed. Special attention is given to the case where there is a remnant pseudo-Nambu-Goldstone resulting from the

\footnotetext{
${ }^{7}$ The cosmological constant problem [79-81] refers to the fact the measured energy density $\rho_{\Lambda} \sim 10^{-48} \mathrm{GeV}^{4}$ [82], related to the cosmological constant by $\Lambda_{C}=8 \pi G_{\text {Newton }} \rho_{\text {vac }}$, is many orders of magnitude smaller than the expected theoretical value.
} 
spontaneous breaking of a global symmetry.

Chapter 3: Localization in the Theory Space. Here we study the inclusion of fermion fields in quiver theories. We obtain the fermion mass hierarchy by different localizations of zero mode fermions in the theory space. In our model [64], this localization does not result in large flavor violation which severely constrains the minimal formulations of Randall-Sundrum models. We also discuss the electroweak precision constraints in quiver models. Furthermore, we derive bounds from the LHC data on the color-singlet and color-octet excited gauge bosons from their decays to jets, bottom and top pairs, and show their dependence on the number of sites in the quiver diagram [68].

Chapter 4: Realizing the Relaxion with $N$-site models. In this chapter, we discuss the relaxion idea, which is a new avenue to explain the radiative stability of the Standard Model Higgs sector. These models require very large field excursions, which are difficult to generate in an UV completion model and to reconcile with the compact field space of the relaxion field. In order to address these issues, we propose in [86] an $N$-site model that generates a large decay constant. The construction involves non-abelian fields, allowing for controlled high energy behavior. This structure admits a continuum limit for a large number of sites, which might be interpreted as a warped extra dimension. 


\section{Chapter 2}

\section{Introduction to Quiver Theories}

\subsection{N-site Models and Large-Scale Hierarchies}

In this section we describe the gauge structure of quiver (or moose) theories $[62,63,87,88]$. The models we are interested in have a linear gauge group structure of the form

$$
G=G_{0} \times G_{1} \times \ldots \times G_{N-1} \times G_{N}
$$

where, in general, $G_{j}=S U(m)_{j}$ is a Yang-Mills symmetry group. For each gauge group there is an associated gauge field $A_{\mu, j}^{a}$, where $a=1,2, \cdots, m^{2}-1$. Furthermore, we add a set of scalar link fields $\Phi_{j}$, which transform in the bi-fundamental representation of $G_{j-1} \times G_{j}$, that is, $\Phi_{j} \rightarrow U_{j-1} \Phi_{j} U_{j}^{\dagger}$. These link fields are an effective description at low energies, and the theory requires an UV completion such as condensates of chiral fermions [62]. In Fig. 2.1, we show a graphical representation for a linear chain model. Each circle represents a site with an associated gauge group $S U(m)_{j}$, and the dashed lines represent the scalar link fields $\Phi_{j}$. The convention is that an outgoing arrow indicates that $\Phi_{j}$ transforms under the fundamental representation of this group, and an ingoing arrow means that $\Phi_{j}$ transforms under the anti-fundamental representation.

In the appropriate limit, this structure can be identified with a compactified warped extra dimension, such that the zeroth site is identified as the ultraviolet (UV) brane, and the $N$-th site as the infra-red (IR) brane. As we shall see, it is possible to establish a dictionary between this purely four-dimensional theory 


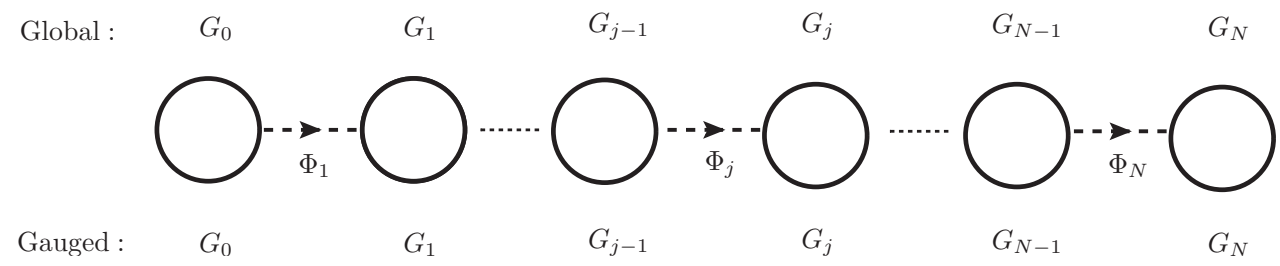

Figure 2.1: Quiver diagram for a linear gauge group chain.

with an extra-dimension model. This means that the fields localized in the UV and IR branes in the Randall-Sundrum models [50,51] can be represented in the deconstructed theory as fields that transform under the groups of zeroth and $N$-th sites, respectively. It is worth emphasizing that the diagram in Fig. 2.1 is only a representation of a four-dimensional theory with a group structure and a specific set of scalar link fields, a priori, the quiver diagram is not interpreted as a physical dimension.

The action for the gauge fields and scalar link fields in this theory is given by

$$
S_{4}^{A, \Phi}=\int d^{4} x\left\{-\frac{1}{2} \sum_{j=0}^{N} \operatorname{Tr}\left[F_{\mu \nu, j} F_{j}^{\mu \nu}\right]+\sum_{j=1}^{N} \operatorname{Tr}\left[\left(D_{\mu} \Phi_{j}\right)^{\dagger} D^{\mu} \Phi_{j}\right]-V\left(\Phi_{j}\right)\right\}
$$

where the traces are over the groups' generators. The covariant derivative is

$$
D_{\mu} \Phi_{j}=\partial_{\mu} \Phi_{j}+i \tilde{g}_{j-1} A_{\mu, j-1 a} T_{j-1}^{a} \Phi_{j}-i \tilde{g}_{j} \Phi_{j} A_{\mu, j a} T_{j}^{a},
$$

where the $T_{j}^{a}$ are the generators of $S U(m)_{j}$ and $\tilde{g}_{j}$ are the associated gauge couplings. The strength tensor $F_{\mu \nu, j}=F_{\mu \nu, j a} T_{j}^{a}$ is written as

$$
F_{\mu \nu, j}^{a}=\partial_{\mu} A_{\nu, j}^{a}-\partial_{\nu} A_{\mu, j}^{a}+\tilde{g}_{j} f_{j}^{a b c} A_{\mu, j b} A_{\nu, j c}
$$

where the $f_{j}^{a b c}$ are the structure constants of the groups, given by $\left[T_{j}^{a}, T_{j}^{b}\right]=i f_{j}^{a b c} T_{j}^{c}$.

If we "turn off" the gauge couplings, the theory has a global symmetry $S U(m)_{j-1} \times$ $S U(m)_{j}$ for each link field. Then, we can recognize this theory as a sum of sigma models (see e.g. [89]). Moreover, we are assuming that the potential for the link fields gives them a vacuum expectation value (vev) which breaks $S U(m)_{j-1} \times S U(m)_{j}$ down to the diagonal group. From these assumptions, we can parametrize the $\Phi_{j}$ 's in the 
non-linear limit as

$$
\Phi_{j}=\frac{f_{j}}{\sqrt{2}} e^{i \sqrt{2} \pi_{j}^{a} \hat{T}^{a} / f_{j}}
$$

where the $\hat{T}^{a}$ are the broken generators, and the $\pi_{j}^{a}$ are the Nambu-Goldstone Bosons (NGB) of the breaking of $S U(m)_{j-1} \times S U(m)_{j}$ to the diagonal group. Each of these breaking happens at the corresponding scale $f_{j}$, which is the vev of the scalar link field $\Phi_{j}$. The vevs of the link fields can be written in the following way [88]

$$
f_{j}=f q^{j}
$$

where $0<q<1$ is a dimensionless constant, and $f$ is an UV mass scale. As a consequence, the $f_{j}$ 's progressively decrease from the zeroth site to the $N$-th site, $f_{1} \cdots>f_{j} \cdots>f_{N}$. In [90] it is shown that it is possible to get the vevs with a decreasing profile without large fine tuning of potential parameters. We also assume that all the gauge groups are identical and that their gauge couplings satisfy

$$
\tilde{g}_{0}(f)=\tilde{g}_{1}\left(f_{1}\right)=\cdots=\tilde{g}_{j}\left(f_{j}\right)=\tilde{g}_{j+1}\left(f_{j+1}\right)=\cdots \equiv \tilde{g}
$$

The parametrization (2.5) allows us to make the matching with the $\mathrm{AdS}_{5}$ models, and the choice (2.6) is the appropriate one to obtain the running of gauge couplings in the continuum limit of the deconstructed theory as they are in the RandallSundrum model [91,92].

When the link fields $\Phi_{j}$ acquire vevs, the global symmetries $S U(m)_{j-1} \times S U(m)_{j}$ break down to the diagonal subgroup $S U(m)_{V_{j}}$. Then, considering the whole chain, there are $N \times\left(m^{2}-1\right)$ NGBs to be absorbed by the gauge bosons that become massive. As we have $N$ link fields for $N+1$ sites, there is a remaining unbroken $S U(m)$ symmetry corresponding to the zero mode gauge boson. This can be seen explicitly if one expands in action (2.1) the following term

$$
\begin{gathered}
\sum_{j=1}^{N} \operatorname{Tr}\left[\left(D_{\mu} \Phi_{j}\right)^{\dagger} D^{\mu} \Phi_{j}\right]=\sum_{j=1}^{N}\left\{\operatorname{Tr}\left[\left(\partial_{\mu} \Phi_{j}\right)^{\dagger} \partial^{\mu} \Phi_{j}\right]+\frac{f_{j}^{2} \tilde{g}^{2}}{2}\left(A_{\mu, j}^{a 2}+A_{\mu, j-1}^{a 2}\right)\right. \\
\left.-2 \tilde{g}^{2} \operatorname{Tr}\left[\Phi_{j}^{\dagger} A_{\mu, j-1} \Phi_{j} A_{j}^{\mu}\right]+\tilde{g} \operatorname{Tr}\left[i\left(\partial^{\mu} \Phi_{j}\right)^{\dagger}\left(A_{\mu, j-1} \Phi_{j}-\Phi_{j} A_{\mu, j}\right)+\text { h.c. }\right]\right\} .
\end{gathered}
$$


Taking the first order term in $\pi_{j} / f_{j}$, we notice that $(2.7)$ can be written as

$$
\mathcal{L}=\sum_{j=1}^{N} \frac{1}{2} \operatorname{Tr}\left[\partial_{\mu} \pi_{j}^{a}-f_{j} \tilde{g}\left(A_{\mu, j}^{a}-A_{\mu, j-1}^{a}\right)\right]^{2}+\cdots
$$

where the dots refer to higher order terms. The mixing between the gauge bosons and the NGBs can be eliminated by adding a gauge fixing term

$$
\mathcal{L}_{\mathrm{GF}}=-\sum_{j=0}^{N} \frac{1}{2 \xi_{j}}\left[\partial_{\mu} A_{j}^{\mu a}+\xi_{j} \tilde{g}\left(f_{j} \pi_{j}^{a}-f_{j+1} \pi_{j+1}^{a}\right)\right]^{2},
$$

where in the following we will take $\xi_{j} \equiv \xi \forall j$. Hence, in the $R_{\xi}$ gauges, the action at the quadratic level in the NGB fields is given by

$$
\begin{aligned}
S_{4}^{A, \pi}=\int d^{4} x\{ & -\frac{1}{2} \sum_{j=0}^{N} \operatorname{Tr}\left[F_{\mu \nu, j} F_{j}^{\mu \nu}\right]-\frac{1}{2 \xi} \sum_{j=0}^{N}\left(\partial_{\mu} A_{j}^{\mu, a}\right)^{2}+\sum_{j=1}^{N} \frac{\tilde{g}^{2} f_{j}^{2}}{2}\left(A_{\mu, j}^{a}-A_{\mu, j-1}^{a}\right)^{2} \\
& \left.+\sum_{j=1}^{N} \frac{1}{2}\left(\partial_{\mu} \pi_{j}^{a}\right)\left(\partial^{\mu} \pi_{j}^{a}\right)-\sum_{j=0}^{N} \frac{\tilde{g}^{2} \xi}{2}\left(f_{j} \pi_{j}^{a}-f_{j+1} \pi_{j+1}^{a}\right)^{2}\right\}
\end{aligned}
$$

The mass term for the NGBs is $[93,94]$

$$
\mathcal{L}_{M_{\pi}}=-\sum_{j=0}^{N} \frac{\xi \tilde{g}^{2}}{2}\left(f_{j} \pi_{j}^{a}-f_{j+1} \pi_{j+1}^{a}\right)^{2} \equiv-\frac{1}{2} \pi^{a t} M_{\pi}^{2} \pi^{a},
$$

where $\pi^{a} \equiv\left(\pi_{1}^{a}, \pi_{2}^{a}, \cdots, \pi_{N}^{a}\right)^{t}$. Therefore, the $N \times N$ mass matrix for the NGBs is given by

$$
M_{\pi}^{2}=\xi \tilde{g}^{2} f^{2}\left(\begin{array}{ccccccc}
2 q^{2} & -q^{3} & 0 & 0 & \ldots & 0 & 0 \\
-q^{3} & 2 q^{4} & -q^{5} & 0 & \ldots & 0 & 0 \\
0 & -q^{5} & 2 q^{6} & 0 & \ldots & 0 & 0 \\
\vdots & \vdots & \vdots & \vdots & \ldots & \vdots & \vdots \\
0 & 0 & 0 & 0 & \ldots & 2 q^{2(N-1)} & -q^{2 N-1} \\
0 & 0 & 0 & 0 & \ldots & -q^{2 N-1} & 2 q^{2 N}
\end{array}\right),
$$

where we used the parametrization in Eq. 2.5. Note that $\operatorname{det}\left(M_{\pi}^{2}\right) \neq 0$, such that in 
the $R_{\xi}$ gauges all the NGBs have non-physical masses which depend on $\sqrt{\xi}$. The unitary gauge is obtained in the limit $\xi \rightarrow \infty$, when the NGBs decouple from the spectrum. This happens because at each site the global symmetry is completely gauged, i. e. the $\left(m^{2}-1\right)$ NGBs from the break of $S U(m)_{j-1} \times S U(m)_{j}$ to the diagonal subgroup are absorbed by the $\left(m^{2}-1\right)$ gauge bosons that become massive. As we mentioned before, there is a remaining unbroken $S U(m)$ symmetry since we have $N$ link fields for $(N+1)$ sites.

Using the unitary gauge, the mass term for the gauge bosons in the Lagrangian (2.1) is given by $[87,88,92]$

$$
\mathcal{L}_{M_{A}}=\frac{\tilde{g}^{2}}{2} \sum_{j=1}^{N}\left[f_{j}\left(A_{\mu, j-1}^{a}-A_{\mu, j}^{a}\right)\right]^{2} .
$$

We can write the $(N+1) \times(N+1)$ matrix $M_{A}^{2}$ for the gauge bosons as

$$
M_{A}^{2}=\tilde{g}^{2} f^{2}\left(\begin{array}{ccccccc}
q^{2} & -q^{2} & 0 & 0 & \ldots & 0 & 0 \\
-q^{2} & q^{2}+q^{4} & -q^{4} & 0 & \ldots & 0 & 0 \\
0 & -q^{4} & q^{4}+q^{6} & -q^{6} & \ldots & 0 & 0 \\
\vdots & \vdots & \vdots & \vdots & \ldots & \vdots & \vdots \\
0 & 0 & 0 & 0 & \ldots & q^{2(N-1)}+q^{2 N} & -q^{2 N} \\
0 & 0 & 0 & 0 & \ldots & -q^{2 N} & q^{2 N}
\end{array}\right)
$$

in the basis $A_{\mu}^{a} \equiv\left(A_{\mu, 0}^{a}, A_{\mu, 1}^{a}, \cdots, A_{\mu, N}^{a}\right)^{t}$. This matrix can be diagonalized by a change of basis $A_{\mu, j}=\sum_{n=0}^{N} f_{j, n} A_{\mu}^{(n)}$, where the $A_{\mu}^{(n)}$ are the mass eigenstates, and we assumed that the group generators are absorbed in the definition of the gauge fields, that is, $A_{\mu, j} \equiv A_{\mu, j}^{a} T^{a}$. Solving this eigensystem, we obtain the following difference equations $[87,88]$

$$
\left(q+q^{-1}-q^{-1}\left(x_{n} q^{-j}\right)^{2}\right) f_{j, n}-q f_{j+1, n}-q^{-1} f_{j-1, n}=0,
$$

where $x_{n} \equiv m_{n} /(\tilde{g} f)$. As one could expect, the determinant of $M_{A}^{2}$ is zero, since there is a remaining unbroken $S U(m)$ symmetry. Thus, the equation (2.13) has solution to $m_{0}=0$, the zero mode gauge boson. Therefore, from (2.13) for the zero mode $(n=0)$ and assuming Neumann "boundary conditions": $f_{0, n}=f_{-1, n}$, and 
$f_{N+1, n}=f_{N, n}$, we conclude that

$$
f_{j+1,0}=f_{j, 0} \quad \forall j .
$$

This means that the zero mode wave-function of the gauge bosons has a flat profile, which is analogous to what happens in warped extra dimensions theories [52-54,95]. Thus, imposing the normalization condition

$$
\sum_{j=0}^{N} f_{j, n}^{2}=1
$$

we obtain the constant $f_{j, 0} \equiv f_{0}$

$$
\sum_{j=0}^{N} f_{0}^{2}=1 \Rightarrow f_{0}=\frac{1}{\sqrt{N+1}}
$$

Equation (2.13) can also be analytically solved for the massive modes [87]. First of all, we define $t[j]=x_{n} q^{-j}$ and $F(t[j])=q^{j} f_{j, n}$. Rewriting (2.13) as a function of these new variables, we get the following difference equation

$$
\left(q+q^{-1}-q^{-1} t^{2}\right) F(t)-F\left(t q^{-1}\right)-F(t q)=0
$$

Equation (2.17) is a special case of the Hahn-Exton equation [96,97]. Its solutions are the so-called $q$-Bessel $J_{\nu}\left(t ; q^{2}\right)$ and $q$-Neumann $Y_{\nu}\left(t ; q^{2}\right)$ functions. These functions are discrete generalizations of Bessel and Neumann functions, and have similar properties to their ordinary ones. Computing the limit $q \rightarrow 1^{-}$of the generalized functions, we get the usual continuous Bessel and Neumann functions [87]. The solution of the difference equation (2.17) is [87]

$$
f_{j, n}=N_{n} q^{-j}\left[J_{1}\left(x_{n} q^{-j} ; q^{2}\right)+\beta\left(x_{n} ; q^{2}\right) Y_{1}\left(x_{n} q^{-j} ; q^{2}\right)\right]
$$

where $N_{n}$ is determined from the wave-function normalization (2.16). $f_{j, n}$ is the coefficient linking the gauge boson at the $j$-site with the mass eigenstate $n$, and from it we can construct the mass eigenstates. Using the boundary conditions for $j=0$ and $j=N$ defined above, we obtain $\beta\left(x_{n} ; q^{2}\right)$ and the gauge boson masses. 
The resulting mass spectrum is given by solutions of [87]

$$
J_{0}\left(x_{n} ; q^{2}\right) Y_{0}\left(x_{n} q^{-(N+1)} ; q^{2}\right)-Y_{0}\left(x_{n} ; q^{2}\right) J_{0}\left(x_{n} q^{-(N+1)} ; q^{2}\right)=0
$$

\subsection{Matching with a Deconstructed Extra-Dimension}

It is interesting to point out that this purely $4 \mathrm{D}$ theory can be obtained from deconstructing an extra-dimensional theory in an AdS background $[87,91,98,99]$. In order to see this explicitly, let us consider a gauge field of a group $G$ in a slice of $\mathrm{AdS}_{5}$ :

$$
d s^{2}=e^{-2 k y} \eta_{\mu \nu} d x^{\mu} d x^{\nu}-d y^{2},
$$

where $y$ is the coordinate in the extra dimension, $k$ is the curvature, and $\eta_{\mu, \nu}=$ $\operatorname{diag}(1,-1,-1,-1)$ is the Minkowski metric in four dimensions. Greek indices refer to four-dimensional coordinates and capital Latin ones refer to both four and five dimension coordinates, such that $M, N=\{0,1,2,3,5\}$. The gauge boson action is written as [100]

$$
\begin{aligned}
S_{5}^{A} & =\int d^{4} x \int_{0}^{\pi R} d y \sqrt{-g}\left\{-\frac{1}{2 g_{5}^{2}} \operatorname{Tr}\left[F_{M N}^{2}\right]\right\} \\
& =\int d^{4} x \int_{0}^{\pi R} d y\left\{-\frac{1}{2 g_{5}^{2}} \operatorname{Tr}\left[F_{\mu \nu} F^{\mu \nu}\right]+\frac{1}{g_{5}^{2}} e^{-2 k y} \operatorname{Tr}\left[\left(\partial_{5} A_{\mu}-\partial_{\mu} A_{5}\right)^{2}\right]\right\},
\end{aligned}
$$

where $g_{5}$ is the 5D gauge coupling. One can discretize the extra dimension by substituting

$$
\begin{aligned}
\int_{0}^{\pi R} d y & \rightarrow \sum_{j=0}^{N} a \\
\partial_{5} A_{\mu} & \rightarrow \frac{A_{\mu, j}-A_{\mu, j-1}}{a}
\end{aligned}
$$

where $a$ is the lattice spacing (inverse of the cutoff). Therefore, one can get: 


$$
S_{5}^{A}=\frac{a}{g_{5}^{2}} \int d^{4} x\left\{-\frac{1}{2} \sum_{j=0}^{N} \operatorname{Tr}\left[F_{\mu \nu, j} F_{j}^{\mu \nu}\right]+\sum_{j=1}^{N} \frac{e^{-2 k a j}}{a^{2}} \operatorname{Tr}\left[\left(A_{\mu, j}-A_{\mu, j-1}-a \partial_{\mu} A_{5, j}\right)^{2}\right]\right\},
$$

where the continuum is obtained in the limit $a \rightarrow 0, N \rightarrow \infty$, keeping fixed $N a=L$, with $L=\pi R$ the size of the extra dimension.

Consider now the $4 \mathrm{D}$ action in (2.1):

$$
S_{4}^{A}=\frac{1}{\tilde{g}^{2}} \int d^{4} x\left\{-\frac{1}{2} \sum_{j=0}^{N} \operatorname{Tr}\left[F_{\mu \nu, j} F_{j}^{\mu \nu}\right]+\sum_{j=1}^{N} f^{2} \tilde{g}^{2} q^{2 j} \operatorname{Tr}\left[\left(A_{\mu, j}-A_{\mu, j-1}-\partial_{\mu} \frac{\pi_{j}}{f_{j}}\right)^{2}\right]\right\}
$$

where for convenience we rescale $A_{\mu, j} \rightarrow A_{\mu, j} / \tilde{g}$ and $\pi_{j}$ is again the Goldstone field transforming in the adjoint of the vector symmetry $S U(m)_{V_{j}}$, which results from the breaking $S U(m)_{j-1} \times S U(m)_{j} \rightarrow S U(m)_{V_{j}}$. This action can be matched with the discretized action in $(2.22)$ by making the following identifications $[62,63,87]^{1}$ :

$$
\begin{aligned}
a & \leftrightarrow \frac{1}{\tilde{g} f} \\
\frac{g_{5}^{2}}{a} & \leftrightarrow \tilde{g}^{2} \\
e^{-k a} & \leftrightarrow q .
\end{aligned}
$$

This matching is not unique and indeed a different identification with varying values of $a_{j}$ and $\tilde{g}_{j}$, and a fixed $f_{j}=f$ for all sites is given in [102]. Notice that the Nambu-Goldstone mode is identified with the scalar component of the gauge field, i.e. the non-linear link field $\Phi_{j} / f_{j}=e^{i \pi_{j} / f_{j}}$ is identified with the Wilson line $\exp \left[i \int_{a j}^{a(j+1)} d y A_{5} e^{-2 k y}\right]$.

It is shown that in the continuum limit, which corresponds to $q \rightarrow 1^{-}$, we obtain the same solutions and mass spectrum of the Kaluza-Klein modes as in warped extra dimensions theories [65]. In this limit, the mass spectrum is given by $m_{n} \sim$ $g f \pi\left(n-\frac{1}{4}\right)(1-q) q^{N}, n>0$. However, in the opposite limit $(q \ll 1)$, the spectrum is exponential, and then just the first massive mode is relevant to the phenomenology. On the other hand, for this $4 \mathrm{D}$ theory to remain an appropriate description of the continuum 5D theory, the $\mathrm{AdS}_{5}$ curvature should satisfy $k<f$. Additionally,

\footnotetext{
${ }^{1}$ See Ref. [101] for a recent exploration of 'Moose/CFT correspondence'.
} 
generating the hierarchy between the Planck and the weak scales while satisfying $k<f$ requires typically that $N>35$, which results in a low energy theory very close to the continuum one. Under these conditions, 4D theories with $k<f$ are just discrete descriptions of theories in $\mathrm{AdS}_{5}$ [87]. On the other hand, here we are interested in the coarse lattice limit, that is, we will consider that the action (2.1) is independent of the continuum 5D theory. In these theories it will be possible to obtain a large hierarchy of scales, as in the Randall-Sundrum theory, using just small values of $N$. For example, if $f \lesssim M_{P}, f_{N} \simeq \mathcal{O}(1) \mathrm{TeV}$, and using $f_{j}=f q^{j}$ (2.5) and the matching (2.26), we can write

$$
e^{-k a N}=\frac{f_{N}}{f} \simeq 10^{-16} \Rightarrow q \simeq 10^{-16 / N}
$$

This choice allows us to solve the gauge hierarchy problem similarly to what is done in the Randall-Sundrum models.

\subsection{A Remnant pseudo-Nambu-Goldstone Boson}

Let us consider now the breaking $G \rightarrow H$ through boundary conditions in theory space, that is, we assume that for the first and last sites, the gauge symmetry group is reduced to $H \subset G$. This is schematically represented in Fig. 2.2

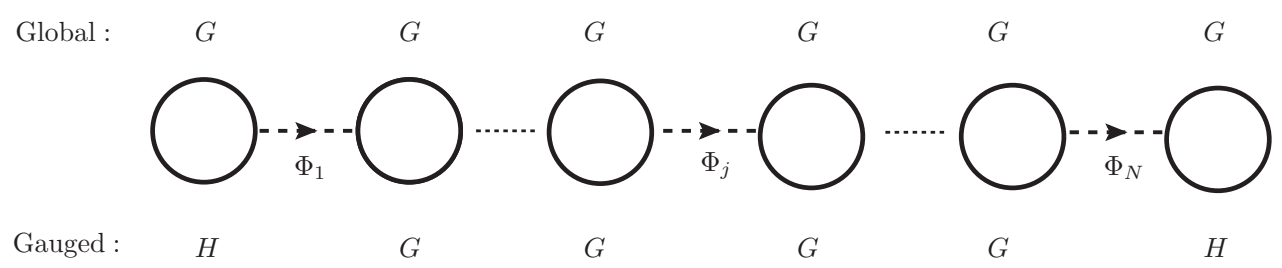

Figure 2.2: Quiver diagram for a linear gauge group chain, where the gauge symmetry is reduced to $H \subset G$ for the sites $j=0$ and $j=N$.

For the broken generators (denoted by hatted indexes), the mixing between the gauge bosons $A_{\mu}^{\hat{a}}$ and the Goldstone modes $\pi_{j}^{\hat{a}}$ is given by 


$$
\begin{aligned}
\mathcal{L} & =\frac{1}{2}\left(\partial_{\mu} \pi_{1}^{\hat{a}}-f_{1} \tilde{g} A_{\mu, 1}^{\hat{a}}\right)^{2}+\sum_{j=2}^{N-1} \frac{1}{2} \operatorname{Tr}\left[\partial_{\mu} \pi_{j}^{\hat{a}}-f_{j} \tilde{g}\left(A_{\mu, j}^{\hat{a}}-A_{\mu, j-1}^{\hat{a}}\right)\right]^{2} \\
& +\frac{1}{2}\left(\partial_{\mu} \pi_{N}^{\hat{a}}+f_{N} \tilde{g} A_{\mu, N-1}^{\hat{a}}\right)^{2}+\cdots
\end{aligned}
$$

where the dots refer to higher order terms. Comparing the expression above with Eq. 2.8, the terms proportional to $\partial_{\mu} \pi_{1} A_{\mu, 0}$ and $\partial_{\mu} \pi_{N} A_{\mu, N}$ do not appear here due to the absence of the corresponding gauge bosons at the borders, as it is illustrated in Fig. 2.2. This happens since in the scenario we discussed in the previous section the whole global group product was gauged. We can remove the mixing between the NG modes and the gauge fields in (2.28) by adding the gauge fixing term:

$$
\mathcal{L}_{\mathrm{GF}}=-\sum_{j=1}^{N-1} \frac{1}{2 \xi}\left[\partial_{\mu} A_{j}^{\mu, \hat{a}}+\xi \tilde{g}\left(f_{j} \pi_{j}^{\hat{a}}-f_{j+1} \pi_{j+1}^{\hat{a}}\right)\right]^{2},
$$

where again we considered the same gauge parameter $\xi$ for all sites. As the gauge symmetry is reduced at the sites $j=0$ and $j=N$, the mass matrix $M_{\pi}^{2}(2.11)$ is now different from the previous case as we explicit show in the following $[93,94]$

$$
\mathcal{L}_{M_{\pi^{\hat{a}}}}=-\sum_{j=1}^{N-1} \frac{\xi \tilde{g}^{2}}{2}\left(f_{j} \pi_{j}^{\hat{a}}-f_{j+1} \pi_{j+1}^{\hat{a}}\right)^{2} \equiv-\frac{1}{2} \pi^{\hat{a} t} M_{\pi^{\hat{a}}}^{2} \pi^{\hat{a}},
$$

where $\pi^{a} \equiv\left(\pi_{1}^{a}, \pi_{2}^{a}, \cdots, \pi_{N}^{a}\right)^{t}$ and the $N \times N$ mass matrix is given by

$$
M_{\pi^{\hat{a}}}^{2}=f^{2} \xi\left(\begin{array}{cccccc}
q^{2} & -q^{3} & 0 & \cdots & 0 & 0 \\
-q^{3} & 2 q^{4} & -q^{5} & \cdots & 0 & 0 \\
0 & -q^{5} & 2 q^{6} & \cdots & 0 & 0 \\
\vdots & \vdots & \vdots & \ddots & \vdots & \vdots \\
0 & 0 & 0 & \cdots & 2 q^{2(N-1)} & -q^{2 N-1} \\
0 & 0 & 0 & \cdots & -q^{2 N-1} & q^{2 N}
\end{array}\right)
$$

Notice that (2.30) differs from Eq. 2.11 only by the limits of the sum. As expected, $\operatorname{Det}\left(M_{\pi^{\hat{a}}}^{2}\right)=0$, indicating the existence of a physical zero mode, i.e. this state 
does not decouple in the unitary gauge limit $(\xi \rightarrow \infty)$. On the other hand, the massive modes have gauge dependent masses and are absorbed to the corresponding gauge bosons in the unitary gauge, which implies that all the $N-1$ gauge bosons associated to the broken generators acquire mass. The eigenstate associated to the null eigenvalue, that is, the one that cannot be removed by a gauge transformation, can be obtained through the eigenvalue equation:

$$
M_{\pi^{\hat{a}}}^{2} \cdot\left(\eta_{1} \pi_{1}^{\hat{a}}, \eta_{2} \pi_{2}^{\hat{a}}, \ldots, \eta_{N} \pi_{N}^{\hat{a}}\right)^{t}=\mathbf{0}
$$

where $\eta_{j}$ 's are weighting the zero mode at each site, i.e. they play the role of the wave-function of the physical state. From the Eq. 2.32, one can obtain the following recursion relation:

$$
\eta_{j}=q \eta_{j+1}
$$

As $0<q<1$, from (2.33) we conclude that the zero mode wave-function is IRlocalized, that is, it is localized towards the site $j=N$. Using the normalization condition $\sum_{j=1}^{N}\left|\eta_{j}\right|^{2}=1$ together with (2.33), one can get that

$$
\eta_{j}=\frac{q^{N-j}}{\sqrt{\sum_{k=1}^{N} q^{2(k-1)}}},
$$

i.e. the physical NGB is

$$
\eta_{\text {phys }}^{\hat{a}}=\sum_{j=1}^{N} \frac{q^{N-j}}{\sqrt{\sum_{k=1}^{N} q^{2(k-1)}}} \pi_{j}^{\hat{a}} .
$$

Note that the fact the physical mode is localized towards the site $j=N$ is a generic feature of this construction, which is independent of the details of the model such as the choice of the gauge groups in the quiver.

Notice that in this construction there is always a parametric scale separation between the pNGBs and the heavy massive states, which is one of the leading motivations for the so-called composite Higgs models [103,104] (see [70] for a review). In this class of theories, the Higgs field is a pNGB appearing in the low energy theory as a result of the spontaneous breaking of a global symmetry $G \rightarrow H$ by a new 
strong sector. A non-trivial potential for the Higgs can be generated for instance by gauging part of this global symmetry, and then explicitly breaking it. The framework implemented in this section follows this same reasoning. As one can notice, in our construction, the pNGB field is dynamically localized close to the IR-site, which is analogous to composite Higgs models in $\mathrm{AdS}_{5}$ [105-108].

Let us remember that this separation of scales is analogous to the mass gap between the pion mass and the masses of other hadrons in QCD. The pions can be understood as NGBs from the spontaneous breaking of the chiral symmetry. As the chiral symmetry is explicitly broken by the up and down masses and by the electromagnetic interaction, the pions are not exact NGBs and acquire their small masses. The apparent parametric separation between the electroweak and the new physics scale motivates models where the Higgs is naturally lighter than the states in the new sector $[109,110]$. 


\section{Chapter 3}

\section{Localization in the Theory Space}

The different localizations of the zero mode fermions in the quiver diagram result in non-universal couplings of them to the massive gauge bosons, which points to the existence of FCNCs (flavor changing neutral currents) at tree level. The FCNC is a challenging problem in Randall-Sundrum models [56-58] as at tree level this effect is absent in the SM, and it results in the most stringent constraint of these theories [59-61]. As we will see, in the quiver theories with few sites the gauge couplings quickly saturate with the localization parameters $\left(c_{L}, c_{R}\right)$, so we can expect to get large mass hierarchy with almost universal couplings. In the next section, we will compute the effective couplings of zero mode fermions to the massive gauge bosons in full-hierarchy quiver theories, and show that it is possible to obtain the quark masses and mixing angles without large tree-level FCNCs.

\subsection{Including the Fermions}

The fermion action in this quiver theory is given by [88]

$$
\begin{array}{r}
S_{\psi}=\int d^{4} x\left\{\sum_{j=0}^{N}\left[\bar{\psi}_{L, j} i \not D_{j} \psi_{L, j}+\bar{\psi}_{R, j} i \not{ }_{j} \psi_{R, j}-\left(\mu_{j} \bar{\psi}_{L, j} \psi_{R, j}+\text { h.c }\right)\right]+\right. \\
\left.-\sum_{j=1}^{N}\left(\lambda_{j} \bar{\psi}_{R, j-1} \Phi_{j} \psi_{L, j}+\text { h.c. }\right)\right\},
\end{array}
$$


where we consider vector-like fermions $\psi_{j}$ transforming in the fundamental representation of $S U(m)_{j}$. The vector-like mass terms and the Yukawa couplings preserve the gauge symmetries. In the most general case of the 4D theory, the Yukawa couplings $\lambda_{j}$ are allowed to be site-dependent. If one wants to get in the continuum limit of this theory the $\mathrm{AdS}_{5}$ theory, it is necessary to take all $\lambda_{j}$ to be equal [88]. The quiver diagram of the theory including the fermions is illustrated in Fig. 3.1. The boundary condition choice $\bar{\psi}_{R, N}=0$ leads to a left-handed zero mode. In order to get a right-handed zero mode in a quiver diagram with the same hopping direction, one needs to remove $\psi_{L, 0}$. The convention is that the outgoing (ingoing) arrows represent chiral fermions in the fundamental (anti-fundamental) representation of $S U(m)$. As in the previous chapter, the link scalar fields $\Phi_{j}$ are $(m, \bar{m})$ under $S U(m)_{j-1} \times S U(m)_{j}$. The dotted lines represent Yukawa couplings of the chiral fermions. In the unitary gauge $\Phi_{j}=f_{j} / \sqrt{2}$, so we write the mass Lagrangian

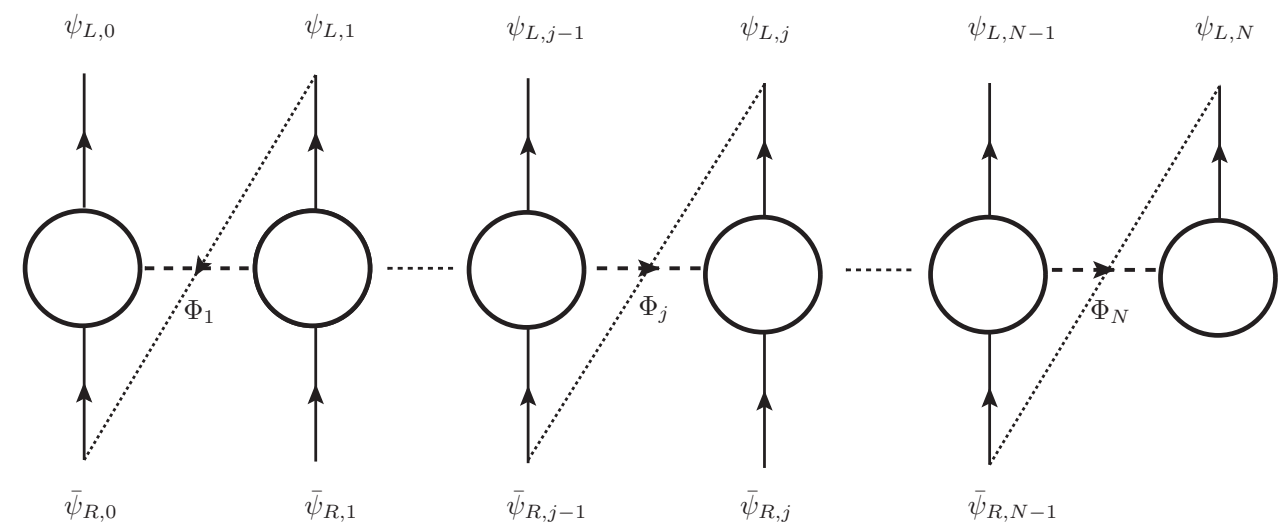

Figure 3.1: Quiver diagram for a linear gauge group chain including the fermions, in the case that the spectrum has a left-handed zero mode fermion.

for the fermions as

$$
\begin{aligned}
\mathcal{L}_{M_{\psi}} & =\sum_{j=1}^{N} \frac{\lambda_{j} f_{j}}{\sqrt{2}}\left(\bar{\psi}_{R, j-1} \psi_{L, j}+\text { h.c. }\right)+\sum_{j=0}^{N}\left(\mu_{j} \bar{\psi}_{L, j} \psi_{R, j}+\text { h.c. }\right) \\
& \equiv \bar{\Psi}_{L} M_{\psi} \Psi_{R}+\text { h.c. }
\end{aligned}
$$


where $\Psi_{L} \equiv\left(\psi_{L, 0}, \psi_{L, 1}, \cdots, \psi_{L, N}\right)^{t}$. Given this, the mass matrix for the left-handed fermions can be written as

$M_{\psi} M_{\psi}^{T}=\left(\begin{array}{cccccc}\mu_{0}^{2} & \frac{\lambda_{1}}{\sqrt{2}} \mu_{0} v_{1} & 0 & \cdots & 0 & 0 \\ \frac{\lambda_{1}}{\sqrt{2}} \mu_{0} v_{1} & \left(\frac{\lambda_{1}}{\sqrt{2}}\right)^{2} v_{1}^{2}+\mu_{1}^{2} & \frac{\lambda_{2}}{\sqrt{2}} \mu_{1} v_{2} & \ldots & 0 & 0 \\ 0 & \frac{\lambda_{2}}{\sqrt{2}} \mu_{1} v_{2} & \left(\frac{\lambda_{2}}{\sqrt{2}}\right)^{2} v_{2}^{2}+\mu_{2}^{2} & \cdots & 0 & 0 \\ \vdots & \vdots & \vdots & \cdots & \vdots & \vdots \\ 0 & 0 & 0 & \cdots & \left(\frac{\lambda_{N-1}}{\sqrt{2}}\right)^{2} v_{N-1}^{2}+\mu_{N-1}^{2} & \frac{\lambda_{N}}{\sqrt{2}} \mu_{N-1} v_{N} \\ 0 & 0 & 0 & \cdots & \frac{\lambda_{N}}{\sqrt{2}} \mu_{N-1} v_{N} & \left(\frac{\lambda_{N}}{\sqrt{2}}\right)^{2} v_{N}^{2}\end{array}\right)$.

As in the gauge bosons case, we can diagonalize this matrix by unitary transformations

$$
\psi_{L, j}=\sum_{n=0}^{N} h_{j, n}^{L} \psi_{L}^{(n)} .
$$

Analogously, it is possible to diagonalize the mass matrix for the right-handed fermions $M_{\psi}^{T} M_{\psi}$ by

$$
\psi_{R, j}=\sum_{n=0}^{N} h_{j, n}^{R} \psi_{R}^{(n)} .
$$

Using the equations of motion for the fields $\psi_{L, j}$ and $\psi_{R, j}$ obtained from the Lagrangian (3.2), and the fact that $\psi_{L}^{(n)}$ and $\psi_{R}^{(n)}$ satisfy the Dirac equation, we obtain

$$
\begin{array}{r}
m_{n} h_{j, n}^{R}+\mu_{j} h_{j, n}^{L}+\frac{\lambda_{j+1}}{\sqrt{2}} f_{j+1} h_{j+1, n}^{L}=0 \\
m_{n} h_{j, n}^{L}+\mu_{j} h_{j, n}^{R}+\frac{\lambda_{j}}{\sqrt{2}} f_{j} h_{j-1, n}^{R}=0,
\end{array}
$$

where $m_{n}$ is the mass of the mass eigenstates $\psi_{L}^{(n)}$ and $\psi_{R}^{(n)}$. Decoupling these equations we get [88]

$$
\begin{array}{r}
\left(\mu_{j}^{2}+\frac{\lambda_{j}^{2} f_{j}^{2}}{2}-m_{n}^{2}\right) h_{j, n}^{L}+\frac{\lambda_{j+1} f_{j+1}}{\sqrt{2}} \mu_{j} h_{j+1, n}^{L}+\frac{\lambda_{j} f_{j}}{\sqrt{2}} \mu_{j-1} h_{j-1, n}^{L}=0 \\
\left(\mu_{j}^{2}+\frac{\lambda_{j+1}^{2} f_{j+1}^{2}}{2}-m_{n}^{2}\right) h_{j, n}^{R}+\frac{\lambda_{j+1} f_{j+1}}{\sqrt{2}} \mu_{j+1} h_{j+1, n}^{R}+\frac{\lambda_{j} f_{j}}{\sqrt{2}} \mu_{j} h_{j-1, n}^{R}=0 .
\end{array}
$$


There are analytical solutions to these equations [87], as in the gauge bosons case. In the continuum limit, the solutions of (3.7) and (3.8) can match the solutions for the eigenfunctions of the Kaluza-Klein fermions in an AdS background [88]. We will not deal with this case here because we are interested in the limit far from the continuum.

Next we handle the zero mode case. From equations (3.5) and (3.6) with $m_{0}=0$, we can obtain the equations of motion for the left-handed zero mode

$$
\begin{aligned}
\mu_{j} h_{j, 0}^{L}+\frac{\lambda_{j+1}}{\sqrt{2}} f_{j+1} h_{j+1,0}^{L} & =0 \Rightarrow \\
& \frac{h_{j+1,0}^{L}}{h_{j, 0}^{L}}=-\frac{\sqrt{2} \mu_{j}}{\lambda_{j+1} f_{j+1}} \equiv q^{c_{L}-1 / 2},
\end{aligned}
$$

and for the right-handed zero mode

$$
\begin{aligned}
\mu_{j} h_{j, 0}^{R}+\frac{\lambda_{j}}{\sqrt{2}} f_{j} h_{j-1,0}^{R} & =0 \Rightarrow \\
& \frac{h_{j, 0}^{R}}{h_{j-1,0}^{R}}=-\frac{\lambda_{j} f_{j}}{\sqrt{2} \mu_{j}} \equiv q^{-\left(c_{R}+1 / 2\right)} .
\end{aligned}
$$

In the left-handed case, for $c_{L}>1 / 2$ the $h_{j, 0}^{L}$ coefficients decrease from the zeroth to the $N$-th site, since $0<q<1$. Therefore, in this case the left-handed zero mode is "localized" close to the zeroth site. However, if $c_{L}<1 / 2$ the left-handed zero mode is "localized" near the $N$-th site. If one identifies the zeroth and $N$-th site as the UV and IR branes of the continuum case, respectively, this behaviour coincides with the left-handed zero mode of the continuum theory [95]. On the other hand, the right-handed zero mode is "localized" near the $N$-th site for $c_{R}>-1 / 2$, and towards the zeroth site for $c_{R}<-1 / 2$. Analogously to the previous case, this behaviour coincides with the right-handed zero mode of the Randall-Sundrum constructions. Moreover, if we choose $j=0$ in the recurrence relations (3.9) and (3.10), we get

$$
\frac{h_{1,0}^{L, R}}{h_{0,0}^{L, R}} \equiv Z_{L, R}
$$

where we defined $Z_{L} \equiv q^{c_{L}-1 / 2}$ and $Z_{R} \equiv q^{-\left(c_{R}+1 / 2\right)}$. By repeating this process, one 
concludes that

$$
h_{j, 0}^{L, R}=h_{0,0}^{L, R}\left(Z_{L, R}\right)^{j} .
$$

Furthermore, using the normalization condition defined by

$$
\sum_{j=0}^{N}\left|h_{j, 0}^{L, R}\right|^{2}=1
$$

we obtain

$$
\sum_{j=0}^{N}\left|h_{j, 0}^{L, R}\right|^{2}=\left|h_{0,0}^{L, R}\right|^{2} \sum_{j=0}^{N}\left(Z_{L, R}^{2}\right)^{j}=1
$$

Computing the geometric series in (3.14) we conclude that

$$
h_{0,0}^{L, R}=\left(\frac{1-Z_{L, R}^{2}}{1-Z_{L, R}^{2(N+1)}}\right)^{1 / 2}
$$

Using the previous results we will compute the Yukawa couplings to the Higgs. For simplicity, we assume that the Higgs only transforms under $S U(m)_{N}$, i.e. the Higgs field is "localized" at the end of the quiver diagram. This assumption will be of little impact for the results of this work. The Yukawa coupling of a fermion to the Higgs localized in the $N$-th site is defined by

$$
\mathcal{L}_{Y}=-Y \bar{\psi}_{R, N} H \psi_{L, N}+\text { h.c. }
$$

where the flavor indices are suppressed and the elementns in the Yukawa matrix $Y$ are assumed to be $\mathcal{O}(1)$ numbers. If we replace in $(3.16)$ the $\bar{\psi}_{R, N}$ and $\psi_{L, N}$ interaction eigenstates (quiver fermions) by their expansions in the mass eigenstates, given by $\bar{\psi}_{R, N}=\sum_{n=0}^{N}\left(h_{N, n}^{R}\right)^{*} \bar{\psi}_{R}^{(n)}$ and $\psi_{L, N}=\sum_{n=0}^{N}\left(h_{N, n}^{L}\right) \psi_{L}^{(n)}$, we obtain for the coupling to the zero mode fermions

$$
\mathcal{L}_{Y}=-Y\left(Z_{L}\right)^{N}\left(Z_{R}\right)^{N}\left(\frac{1-Z_{L}^{2}}{1-Z_{L}^{2(N+1)}}\right)^{1 / 2}\left(\frac{1-Z_{R}^{2}}{1-Z_{R}^{2(N+1)}}\right)^{1 / 2} \bar{\psi}_{R}^{(0)} H \psi_{L}^{(0)}+\text { h.c. }
$$

where we used (3.12) and (3.15). Thus, the effective Yukawa coupling of the Higgs 
to the zero mode fermions is given by

$$
Y_{e f f}=Y\left(Z_{L}\right)^{N}\left(Z_{R}\right)^{N}\left(\frac{1-Z_{L}^{2}}{1-Z_{L}^{2(N+1)}}\right)^{1 / 2}\left(\frac{1-Z_{R}^{2}}{1-Z_{R}^{2(N+1)}}\right)^{1 / 2} .
$$

It is possible to obtain the fermion mass hierarchy even if we use non-hierarchical Yukawa couplings in $Y$. This can be done by choosing the appropriate localization of the zero modes fermions in the quiver diagram, i.e. the $c_{L}$ and $c_{R}$ parameters. Therefore, the large SM fermion mass hierarchy can be explained by the different zero modes' localizations. This way of obtaining the hierarchical masses is very similar to what is done in the Randall-Sundrum theory. As we shall show in the next section, this can be achieved in quiver theories with just few sites, i.e. in models very far from the continuum limit. To explore quantitatively the flavor violation and the electroweak precision constraints we choose $N=4$, but any model with few sites will have the same qualitative features. For instance, we show a solution satisfying the electroweak precision bounds that has the SM quark masses and the CKM mixing matrix without large FCNCs at tree level.

\subsection{Gauge Couplings}

In the deconstructed theory given by action (3.1), the coupling of the gauge bosons to the left-handed zero mode fermions is written as

$$
\mathcal{L}_{\psi A}=\sum_{j=0}^{N} \tilde{g}_{j} \bar{\psi}_{L, j} A_{\mu, j} \gamma^{\mu} \psi_{L, j}
$$

where $\tilde{g}_{j}$ is the gauge coupling associated with the $S U(m)_{j}$ gauge group, and $\psi_{L, j}$, $A_{\mu, j}$ are interaction eigenstates, and the group generators are absorbed in the definition of the gauge fields. As explained previously in Sec. 2.1, we assume that the gauge coupling is universal, that is, $\tilde{g}_{j}=\tilde{g}$ for all $j$. If we expand in the Lagrangian (3.18) the fields in the mass eigenstate bases, we obtain

$$
\mathcal{L}_{\psi A}=\sum_{j, n, m, p=0}^{N}\left[\tilde{g}\left(h_{j, n}^{L}\right)^{*} f_{j, m} h_{j, p}^{L}\right] \bar{\psi}_{L}^{(n)} A_{\mu}^{(m)} \gamma^{\mu} \psi_{L}^{(p)}
$$


Therefore, the effective coupling of the gauge bosons to the left-handed fermions in quiver theories is

$$
g_{n m p}^{L}=\sum_{j, n, m, p=0}^{N}\left[\tilde{g}\left(h_{j, n}^{L}\right)^{*} f_{j, m} h_{j, p}^{L}\right] .
$$

An analogous expression is obtained for the right-handed fermions. In order to relate the $\tilde{g}$ couplings to the Standard Model ones, we use the effective coupling of the zero mode gauge boson $(m=0)$ to the zero mode fermions $(n, p=0)$. Thus, for the leftand right- handed cases, we obtain

$$
g_{00}^{L, R}=\frac{\tilde{g}}{\sqrt{N+1}} \sum_{j=0}^{N}\left|h_{j, 0}^{L, R}\right|^{2}=\frac{\tilde{g}}{\sqrt{N+1}},
$$

where we use the normalization conditions for $h_{j, 0}^{L, R}(3.13)$, and the fact that the wavefunction for the zero mode gauge boson has a flat profile, that is, $f_{j, 0}=1 / \sqrt{N+1}$ (2.16). As the zero mode gauge couplings $\left(g_{00}^{L, R}\right)$ must be the Standard Model couplings, we get the general relation

$$
\tilde{g}^{\left({ }^{\prime}\right)}=\sqrt{N+1} g^{\left({ }^{\prime}\right)}
$$

where $g$ and $g^{\prime}$ are the usual gauge couplings of the $W_{a}^{\mu}$ and $B^{\mu}$ bosons, respectively.

In order to obtain consequences for the Standard Model fermion sector, we focus on the couplings to the zero mode fermions. Furthermore, we are interested in obtaining the coupling of zero mode fermions to the first excited gauge boson, since this state gives the largest contribution to the FCNCs. Thus, from (3.20) this coupling is given by

$$
g_{01}^{L}=\sum_{j=0}^{N} \tilde{g}\left|h_{j, 0}^{L}\right|^{2} f_{j, 1} .
$$

The coefficients $f_{j, 1}$ can be obtained by diagonalizing the gauge bosons matrix following the procedure described in Sec. 2.1, and the expression for $h_{j, 0}^{L}$ is given by (3.12). Using these expressions, one finds that

$$
g_{01}^{L}=\sum_{j=0}^{N} \tilde{g}\left(Z_{L}\right)^{2 j}\left(\frac{1-Z_{L}^{2}}{1-Z_{L}^{2(N+1)}}\right) f_{j, 1},
$$


where $Z_{L}=q^{c_{L}-1 / 2}$. The procedure to obtain the coupling of the right-handed zero mode fermions to the first massive mode of a gauge boson is analogous to the one performed to the left-handed zero mode fermions. The resulting gauge coupling is given by

$$
g_{01}^{R}=\sum_{j=0}^{N} \tilde{g}\left(Z_{R}\right)^{2 j}\left(\frac{1-Z_{R}^{2}}{1-Z_{R}^{2(N+1)}}\right) f_{j, 1},
$$

where $Z_{R}=q^{-\left(c_{R}+1 / 2\right)}$. Fig. 3.2 shows the couplings of the left-handed zero mode fermions to the first excited state of a gauge boson as a function of the localization parameter $c_{L}$ for theories with different numbers of sites. The couplings are normalized by the SM gauge coupling $g$.

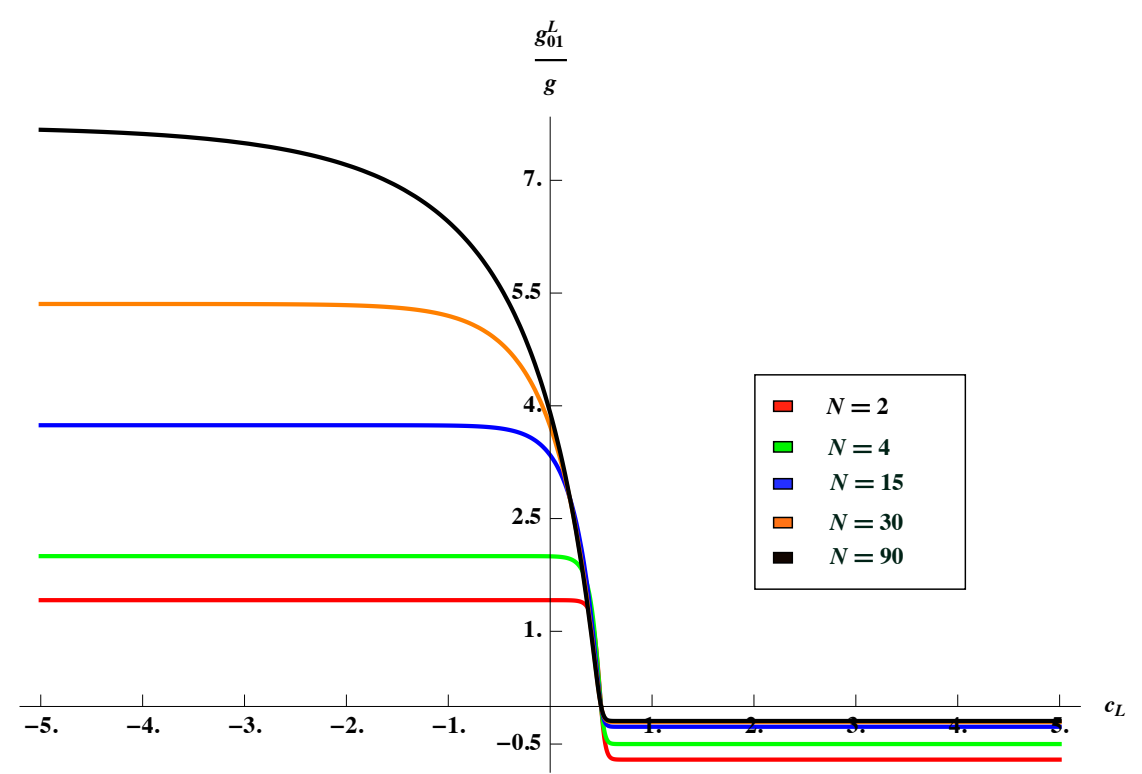

Figure 3.2: Couplings of left-handed zero mode fermions to the first massive mode of a gauge boson (normalized to the zero mode gauge boson coupling) as a function of the localization parameter $c_{L}$. For the left side of the plot and starting from the bottom: $N=2, N=4, N=15$, $N=30$ and $N=90$.

In Fig. 3.2 we observe that the effective gauge couplings have different behaviours for theories with few sites when compared with theories near to the continuum limit, i.e. models with large values of $N$. Furthermore, we see that as $N$ increases, the couplings go to their continuum limit, as it is possible to verify by comparing the $N=90$ case with the results for Randall-Sundrum models in [95]. In fact, as 
discussed before, using the appropriated matchings, this desconstructed theory in the continuum limit coincides with a 5D theory with a curved extra dimension in an AdS background $[87,88]$. It is interesting to note that for the models with few sites, the gauge couplings quickly saturate with the localization, the $c_{L}$ parameter, so in these cases there are two well-defined plateaus. Therefore, as we know the flavor violation is a consequence of the non-universality of couplings, we can expect that a solution for the fermion masses and mixings such that all localization parameters are in the same plateau implies suppressed flavor violation at tree level. The same conclusion is obtained for the right-handed couplings. In the following, we study the parameters space for a quiver theory with $N=4$, and show solutions that have the Standard Model quark masses and the CKM mixing matrix without large FCNCs at tree level.

\subsection{Model with a Few Sites}

We chose a model with five sites $(N=4)$ in order to have quantitative results, but any model with few sites will have similar features. The solutions satisfying the quark masses and the mixings angles were found by a scan of the parameter space using a genetic algorithm. Our purpose here is not to perform a detailed study of the parameter space, but point out cases that represent different classes of solutions.

The Fig. 3.3 shows the coupling of the left-handed zero mode fermions to the first excitation of a gauge boson. In this plot there are two plateaus for which the couplings are universal: one above $c_{L} \gtrsim 1 / 2$, and other for $c_{L} \lesssim 0.25$. The lower plateau corresponds to localization close to the zeroth site, that is, in the UV region. For the fermions in the upper plateau the localization is close to the $N$-th site, i.e. near the IR. In the Randall-Sundrum model there is just the UV plateau [95], which means that in the continuum case we can obtain universal couplings only for localizations in the UV. In our model with five sites we expect that a given solution for the $c_{L}$ 's in which they are in the same plateau will have very small flavor violation. This is analogous for the right-handed zero mode fermions. Summarizing, we want to find solutions for the localization parameters $c_{L, R}^{i}$ 's, where $i=1,2,3$ denotes generation, which are as much as possible on the plateaus in order to minimize flavor violation. We divide the solutions in two classes which have different features, 


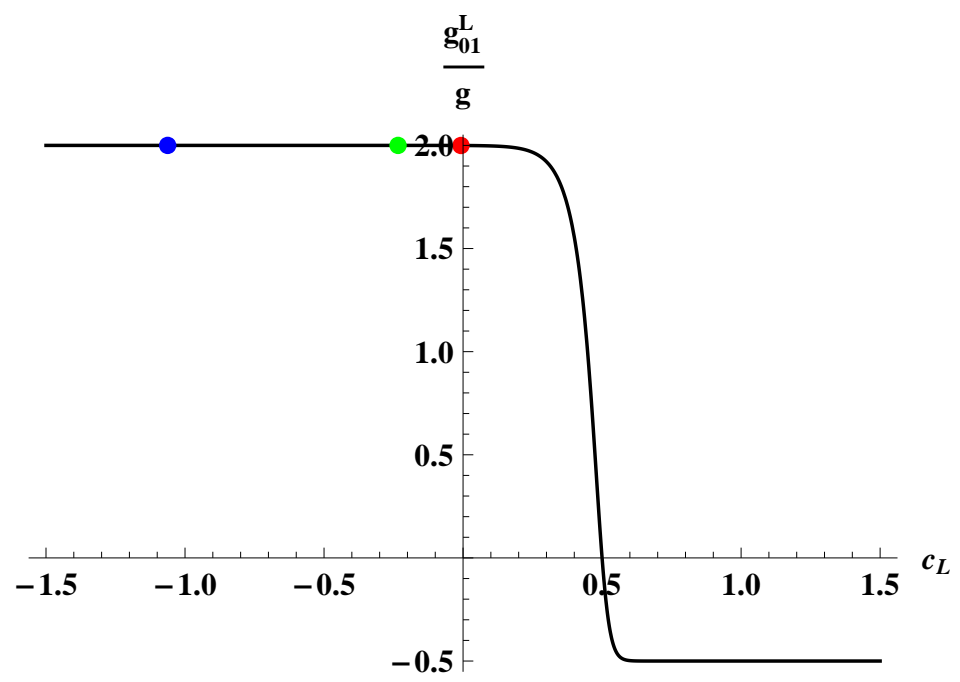

Figure 3.3: Couplings of the left-handed doublet zero mode quarks to the first excited state of a gauge boson for $N=4$ (in units of the zero mode gauge coupling) as a function of the localization parameter $c_{L}$. The dots show the localization for case $\mathbf{A}$.

called case $\mathbf{A}$ and case $\mathbf{B}$.

In case A (Figs. 3.3, 3.4, and 3.5) the right-handed quark sector is localized towards the UV plateau, while the left-handed quark sector is localized in the IR plateau. The dots indicate a solution for the quark sector in the interaction (diagonal) basis that results in the correct masses and mixing angles. We can see that the couplings of the left-handed doublet zero mode quarks (Fig. 3.3) and right-handed zero mode down quarks (Fig. 3.4) are universal, so in these cases there is no flavor violation. The only exception is the up-type right-handed quark sector, which does not have universal couplings to the first gauge boson excitation, as shown in Fig. 3.5. This happens because to generate the large top quark mass the right-handed top needs to be closer to the IR in order to enhance the coupling with Higgs, which results in a source of tree-level flavor violation for case A. 


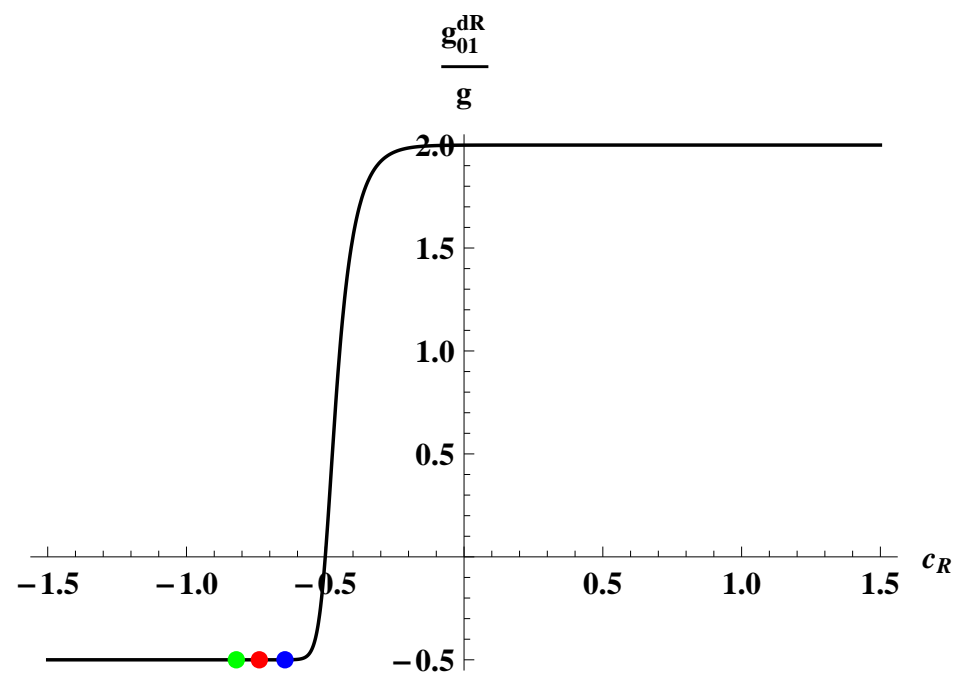

Figure 3.4: Couplings of the right-handed zero mode down quarks to the first excited state of a gauge boson for $N=4$ (in units of the zero mode gauge coupling) as a function of the localization parameter $c_{R}$. The dots show the localization for case $\mathbf{A}$.

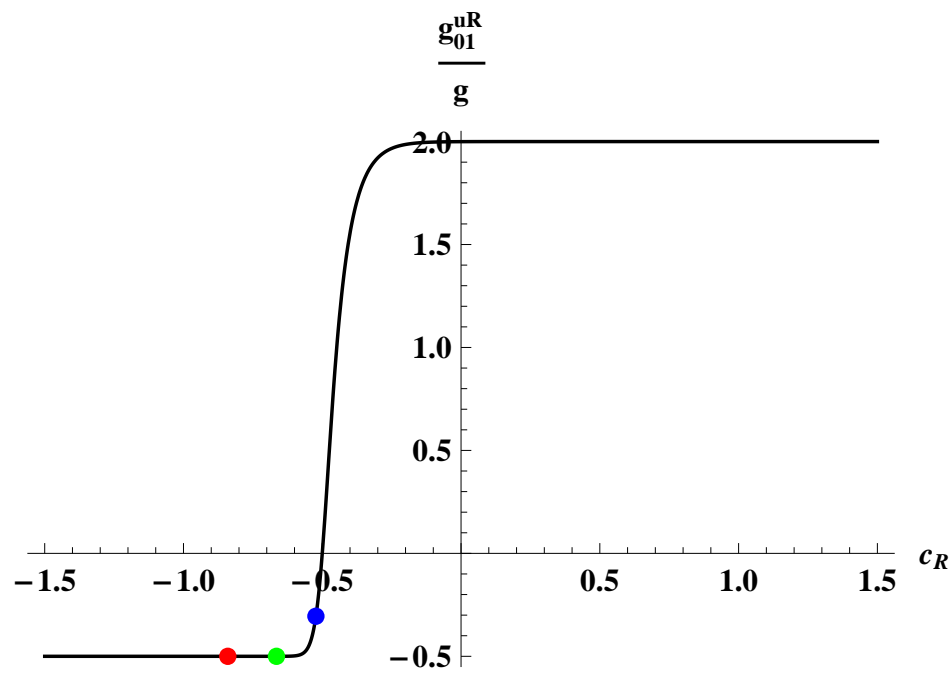

Figure 3.5: Couplings of the right-handed zero mode up quarks to the first excited state of a gauge boson for $N=4$ (in units of the zero mode gauge coupling) as a function of the localization parameter $c_{R}$. The dots show the localization for case $\mathbf{A}$. 


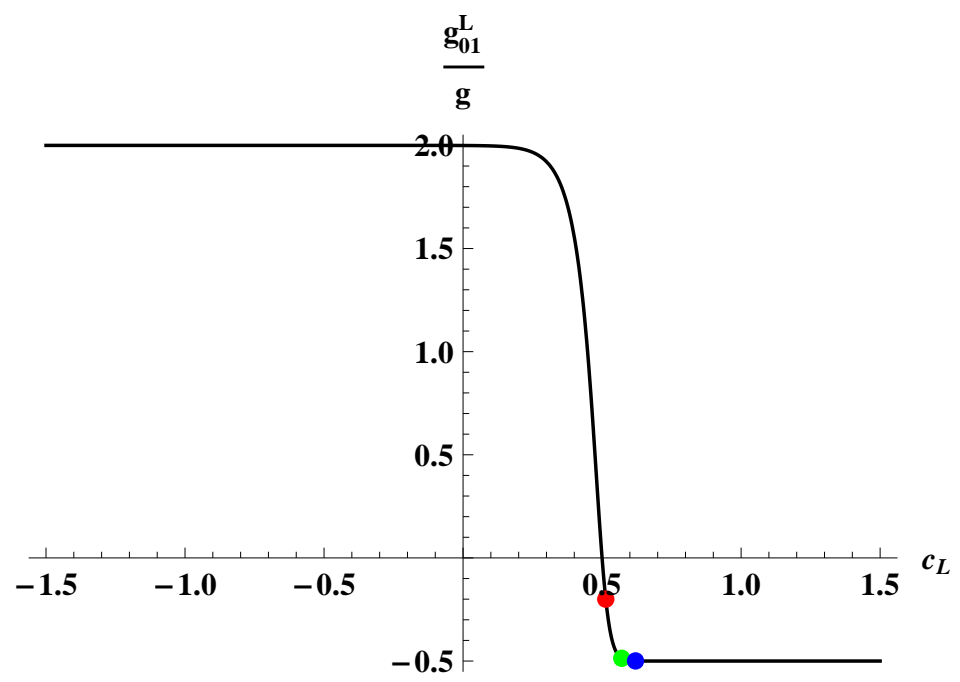

Figure 3.6: Couplings of the left-handed doublet zero mode quarks to the first excited state of a gauge boson for $N=4$ (in units of the zero mode gauge coupling) as a function of the localization parameter $c_{L}$. The dots show the localization for case $\mathbf{B}$.

A solution for case B is shown in Figs. 3.6, 3.7, and 3.8. The main difference in this case with respect to the previous one is that now the left-handed zero mode quarks are localized towards the UV, Fig. 3.6. The down-type right-handed quarks remain universally coupled to the first excited gauge boson in the UV plateau, Fig. 3.7. The right-handed up sector in this case is also localized towards the UV, and again it does not have universal couplings to the first massive gauge boson since one dot is localized closer to the IR to generate the large top mass. Comparing with case A, here there is more flavor violation because besides the right-handed up sector there is also flavor violation in the left-handed sector. In particular, the nonuniversality of the doublets' couplings introduces flavor violation in the down-type quarks. However, as we will see, this effect is still lower than in the continuum case. 


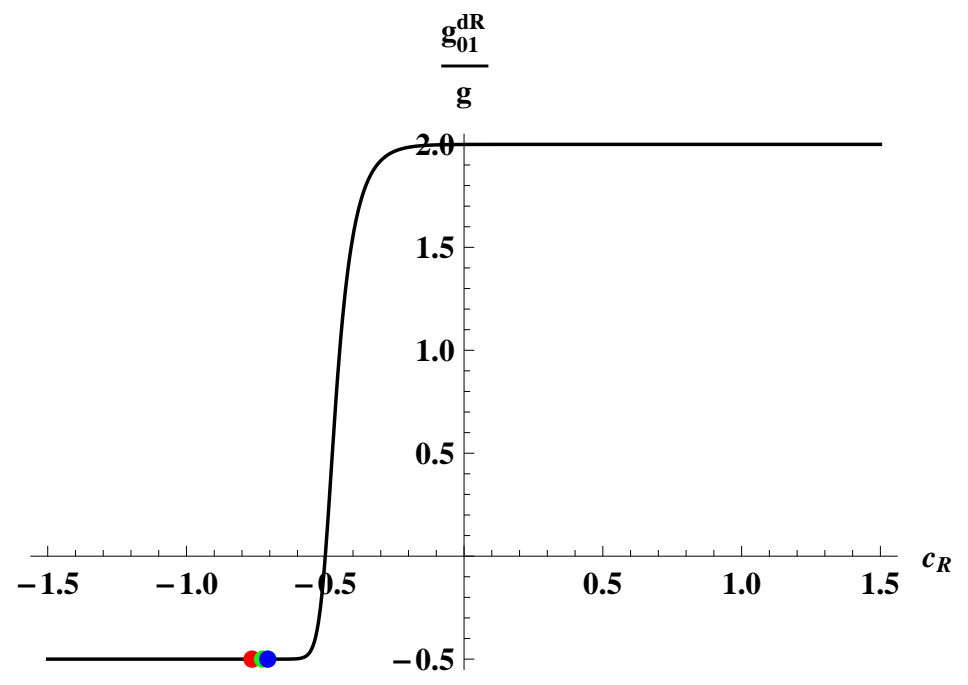

Figure 3.7: Couplings of the right-handed zero mode down quarks to the first excited state of a gauge boson for $N=4$ (in units of the zero mode gauge coupling) as a function of the localization parameter $c_{R}$. The dots show the localization for case $\mathbf{B}$.

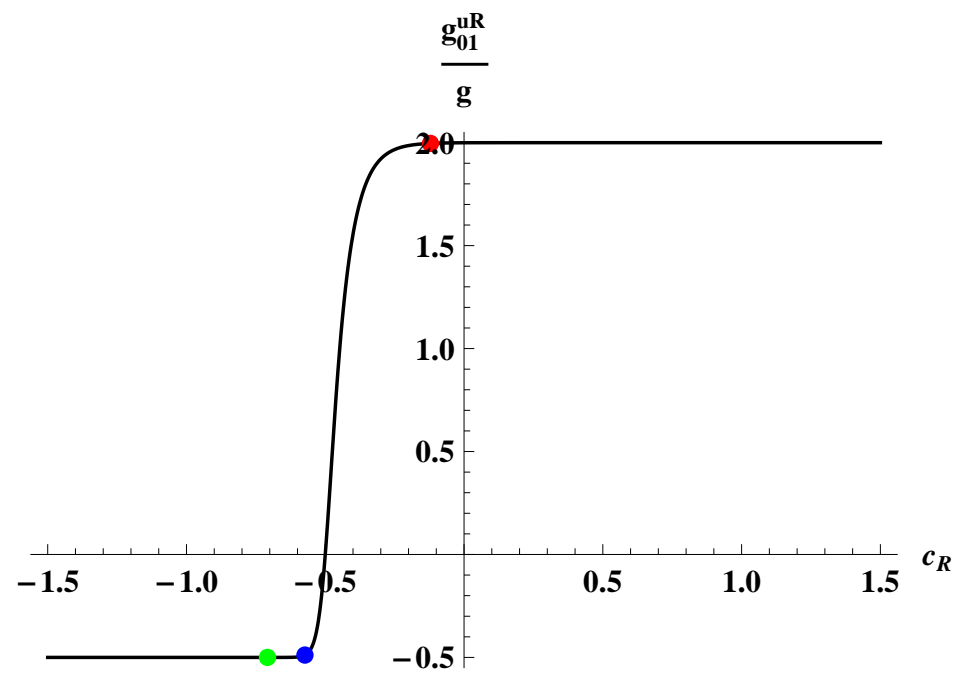

Figure 3.8: Couplings of the right-handed zero mode up quarks to the first excited state of a gauge boson for $N=4$ (in units of the zero mode gauge coupling) as a function of the localization parameter $c_{R}$. The dots show the localization for case $\mathbf{B}$.

\subsubsection{Flavor Violation Bounds}

In this subsection we obtain the flavor violation bounds resulting from the treelevel couplings of the zero mode fermions to the first excited state of a gauge boson. 
In our model, it is not necessary to assume the strong interaction in the quiver diagram since we want to solve the hierarchy problems associated to the electroweak gauge group sector. However, we will consider the case when $S U(3)_{c}$ propagates in the quiver, because its consequences generate the most stringent constraints. Furthermore, this case allows us to make a comparison with the analogous flavor violation bounds in the Randall-Sundrum models.

In order to study quantitatively the flavor violation effects we must rotate the couplings to the mass eigenstate basis. Remember that in Sec. 3.3 the couplings of quarks to the first gauge excitation (Figs. 3.3-3.8) are in the interaction (diagonal) basis. We define the couplings of the up-type left-handed quarks in the mass eigenstate basis as

$$
G_{L}^{U} \equiv U_{L}^{-1}\left(\begin{array}{ccc}
g_{u_{L}} & 0 & 0 \\
0 & g_{c_{L}} & 0 \\
0 & 0 & g_{t_{L}}
\end{array}\right) U_{L}
$$

where $U_{L}$ is a unitary matrix that rotates to the mass eigenstates, and the couplings $g_{u_{L}}, g_{c_{L}}$, and $g_{t_{L}}$ are computed in (3.24), which are indicated by the dots in Fig. 3.3 for case A, and Fig. 3.6 for case B. Similarly, we define rotation matrices for the right-handed up quarks, as well as for left- and right-handed down sector. The nondiagonal values in the $G$ 's matrices lead to tree-level flavor violation, which results in bounds on the mass of the first gluon excitation in full-hierarchy quiver theories (FHQT). These non-diagonal couplings are shown in Tabs. 3.1 and 3.2 for the case A and case B, respectively.

\begin{tabular}{|c|c|c|c|c|c|}
\hline & $\mathrm{L}$ & $\mathrm{R}$ & & $\mathrm{L}$ & $\mathrm{R}$ \\
\hline$\left|G^{u, c}\right|$ & $1.1 \times 10^{-5}$ & $2.2 \times 10^{-8}$ & $\left|G^{d, s}\right|$ & $5.7 \times 10^{-5}$ & $1.6 \times 10^{-9}$ \\
\hline$\left|G^{u, t}\right|$ & $2.0 \times 10^{-4}$ & $2.3 \times 10^{-6}$ & $\left|G^{d, b}\right|$ & $1.9 \times 10^{-4}$ & $2.1 \times 10^{-8}$ \\
\hline$\left|G^{c, t}\right|$ & $5.5 \times 10^{-6}$ & $6.8 \times 10^{-4}$ & $\left|G^{s, b}\right|$ & $5.9 \times 10^{-5}$ & $2.5 \times 10^{-6}$ \\
\hline
\end{tabular}

Table 3.1: Non-diagonal values of the quark couplings to the first excited state of the gluon in case A. 


\begin{tabular}{|c|c|c|c|c|c|}
\hline & $\mathrm{L}$ & $\mathrm{R}$ & & $\mathrm{L}$ & $\mathrm{R}$ \\
\hline$\left|G^{u, c}\right|$ & $2.8 \times 10^{-3}$ & $2.9 \times 10^{-4}$ & $\left|G^{d, s}\right|$ & $5.7 \times 10^{-4}$ & $6.5 \times 10^{-6}$ \\
\hline$\left|G^{u, t}\right|$ & $4.2 \times 10^{-3}$ & $2.9 \times 10^{-3}$ & $\left|G^{d, b}\right|$ & $5.9 \times 10^{-3}$ & $5.0 \times 10^{-5}$ \\
\hline$\left|G^{c, t}\right|$ & $3.3 \times 10^{-2}$ & $1.8 \times 10^{-1}$ & $\left|G^{s, b}\right|$ & $6.7 \times 10^{-3}$ & $1.2 \times 10^{-4}$ \\
\hline
\end{tabular}

Table 3.2: Non-diagonal values of the quark couplings to the first excited state of the gluon in case B.

At low energies, the excited gluon is integrated out and the effective Hamiltonian for $\Delta F=2$ transitions receives contributions given by

$$
\begin{aligned}
H_{\mathrm{eff}}= & \frac{1}{M_{G}^{2}}\left[\frac{1}{6} G_{L}^{i j} G_{L}^{i j}\left(\bar{q}_{L}^{i \alpha} \gamma^{\mu} q_{L \alpha}^{j}\right)\left(\bar{q}_{L}^{i \beta} \gamma^{\mu} q_{L \beta}^{j}\right)+(\mathrm{L} \leftrightarrow \mathrm{R})\right. \\
& \left.-G_{L}^{i j} G_{R}^{i j}\left(\left(\bar{q}_{R}^{i \alpha} q_{L \alpha}^{j}\right)\left(\bar{q}_{L}^{i \beta} q_{R \beta}^{j}\right)-\frac{1}{3}\left(\bar{q}_{R}^{i \alpha} q_{L \beta}^{j}\right)\left(\bar{q}_{L}^{i \alpha} q_{R \beta}^{j}\right)\right)\right] .
\end{aligned}
$$

Therefore, the coefficients in (3.26) are identified as the corresponding Wilson coefficients of the low energy $\Delta F=2$ Hamiltonian. Using [111], the contributions from the Hamiltonian (3.26) are

$$
C_{M}^{1}\left(M_{G}\right)=\frac{1}{6} \frac{\left(G_{L}^{i j}\right)^{2}}{M_{G}^{2}}, \quad C_{M}^{4}\left(M_{G}\right)=\frac{G_{L}^{i j} G_{R}^{i j}}{M_{G}^{2}} \quad C_{M}^{5}\left(M_{G}\right)=\frac{G_{L}^{i j} G_{R}^{i j}}{3 M_{G}^{2}},
$$

where $M=K, D, B_{d}, B_{s}$ indicates different mesons. It is possible to obtain constraints for $M_{G}$ using the Wilson coefficients bounded by the UTFit collaboration fits for the flavor data $[111,112]$. These bounds are obtained at larger scales $\Lambda$, and when $\Lambda$ is much larger than $M_{G}$, the correction coming from the renormalization group evolution of the Wilson coefficients is significant, and must be considered. The results are shown in Tab. 3.3. The third column gives the bounds on the scale of New Physics (NP) implied by assuming $C(\Lambda) \sim 1 / \Lambda^{2}$. On the other hand, in our model the Wilson coefficients (3.27) have a natural large suppression, and thus the scale $M_{G}$ can be much lower as it is shown in the fourth column for case B.

Comparing the Tabs. 3.1 and 3.2, we see that the couplings in case A are much smaller than the ones in case B, and the former will not result in significant flavor violation bounds. Case A minimizes the tree-level flavor violation effects, but as we will see in the next section, the zero mode fermion localizations in this case will result 


\begin{tabular}{cccc}
\hline \hline Parameter & $\begin{array}{c}95 \% \text { allowed range } \\
\left(\mathrm{GeV}^{-2}\right)\end{array}$ & $\begin{array}{c}\text { Lower limit on } \Lambda(\mathrm{TeV}) \\
\text { for arbitrary NP }\end{array}$ & $\begin{array}{c}\text { Bound on Color-octect } \\
\text { Mass in FHQT }(\mathrm{TeV})\end{array}$ \\
\hline $\operatorname{Re} C_{K}^{1}$ & {$[-9.6,9.6] \cdot 10^{-13}$} & $1.0 \cdot 10^{3}$ & 0.2 \\
$\operatorname{Re} C_{K}^{4}$ & {$[-3.6,3.6] \cdot 10^{-15}$} & $17 \cdot 10^{3}$ & 0.1 \\
$\operatorname{Re} C_{K}^{5}$ & {$[-1.0,1.0] \cdot 10^{-14}$} & $10 \cdot 10^{3}$ & 0.1 \\
\hline $\operatorname{Im} C_{K}^{1}$ & {$[-2.6,2.8] \cdot 10^{-15}$} & $1.9 \cdot 10^{4}$ & 2.6 \\
$\operatorname{Im} C_{K}^{4}$ & {$[-4.1,3.6] \cdot 10^{-18}$} & $49 \cdot 10^{4}$ & 3.0 \\
$\operatorname{Im} C_{K}^{5}$ & {$[-1.2,1.1] \cdot 10^{-17}$} & $29 \cdot 10^{4}$ & 1.0 \\
\hline \hline$\left|C_{D}^{1}\right|$ & $<7.2 \cdot 10^{-13}$ & $1.2 \cdot 10^{3}$ & 1.0 \\
$\left|C_{D}^{4}\right|$ & $<4.8 \cdot 10^{-14}$ & $4.6 \cdot 10^{3}$ & 2.9 \\
$\left|C_{D}^{5}\right|$ & $<4.8 \cdot 10^{-13}$ & $1.4 \cdot 10^{3}$ & 0.5 \\
\hline \hline$\left|C_{B_{d}}^{1}\right|$ & $<2.3 \cdot 10^{-11}$ & $0.21 \cdot 10^{3}$ & 0.3 \\
$\left|C_{B_{d}}^{4}\right|$ & $<2.1 \cdot 10^{-13}$ & $2.2 \cdot 10^{3}$ & 0.3 \\
$\left|C_{B_{d}}^{5}\right|$ & $<6.0 \cdot 10^{-13}$ & $1.3 \cdot 10^{3}$ & 0.1 \\
\hline \hline$\left|C_{B_{s}}^{1}\right|$ & $<1.1 \cdot 10^{-9}$ & 30 & 0.1 \\
$\left|C_{B_{s}}^{4}\right|$ & $<1.6 \cdot 10^{-11}$ & 250 & 0.1 \\
$\left|C_{B_{s}}^{5}\right|$ & $<4.5 \cdot 10^{-11}$ & 150 & 0.03 \\
\hline \hline
\end{tabular}

Table 3.3: $95 \%$ probability range for $C(\Lambda)$ and the corresponding lower bounds on the NP scale $\Lambda$ for arbitrary NP flavor structure [111,112]. The last column corresponds to the bound on the gluon excitation in full-hierarchy quiver theories (FHQT) in case B.

in larger contributions to the electroweak parameters. Nevertheless, in case B, the zero mode fermion localizations minimize the effects on the $S$ and $T$ parameters, but there is larger flavor violation at tree level. As we see in Tab.3.3, the most stringent constraint comes from kaon physics, the bound on $\operatorname{Im} C_{K}^{4}$, which results in $M_{G}>3 \mathrm{TeV}$. This bound represents the typical value in agreement with all flavor constraints for the solution called case B, and it is not necessarily the smallest possible value for $M_{G}$ for all solutions.

To sum up, in quiver models with few sites it is possible to obtain the quark masses and mixings without large FCNCs at tree level. This is not the case in Randall-Sundrum models, where the typical tree-level flavor violation in the downquark sector leads to challenging bounds. For instance, to overcome the kaon physics constraints without introducing new global flavor symmetries these models require first excited state masses of at least $M_{\mathrm{KK}} \sim 20 \mathrm{TeV}$ [59-61], which generates a hierarchy between the first massive state and the electroweak scale. 


\subsubsection{Electroweak Precision Parameters}

The electroweak precision measurements [113] set important bounds for the physics beyond the Standard Model. We compute the contribution of our model to the $S$ and $T$ parameters [114] that can be defined using the Standard Model vacuum polarization functions for the electroweak sector as

$$
\begin{aligned}
S & =\frac{16 \pi}{g^{2}+g^{\prime 2}}\left[\Pi_{Z Z}^{\prime}(0)-\frac{g^{2}-g^{2}}{g g^{\prime}} \Pi_{\gamma Z}^{\prime}(0)-\Pi_{\gamma \gamma}^{\prime}(0)\right] \\
& =16 \pi\left[\Pi_{33}^{\prime}(0)-\Pi_{3 Q}^{\prime}(0)\right], \\
T & =\frac{4 \pi}{e^{2}}\left[\frac{\Pi_{W W}(0)}{M_{W}^{2}}-\frac{\Pi_{Z Z}(0)}{M_{Z}^{2}}\right], \\
& =\frac{16 \pi}{v_{\mathrm{EW}}^{2} e^{2}}\left[\Pi_{11}(0)-\Pi_{33}(0)\right] .
\end{aligned}
$$

In full-hierarchy quiver theories, as in the Randall-Sundrum models, there are contributions to $S$ and $T$ parameters already at tree level. These come from the mixing of the Standard Model gauge bosons $W^{ \pm}$and $Z$ with their excited states by the Higgs vev insertions, as it is shown in Figs. 3.9 and 3.10. Additionally, there are contributions coming from the universal shifts in the gauge couplings of light fermions, the mixing is represented in Fig. 3.11. The $S$ parameter does not receive significant contributions from diagrams as shown in Figs. 3.9 and 3.10, since these are suppressed by a factor of $v_{\mathrm{EW}}^{4} / M_{1}^{4}$, where $M_{1}$ is the mass of the first gauge boson excitation.

In this five sites model we assume that the Higgs doublet is localized in the $N$-th site. We also use the minimum choice for the electroweak sector in the quiver, that is, $S U(2)_{L} \times U(1)_{Y}$ for all sites. The analogous situation in a curved extra-dimensional theory would be to have the Standard Model gauge fields propagating in the bulk, and the Higgs doublet localized in the IR brane. 


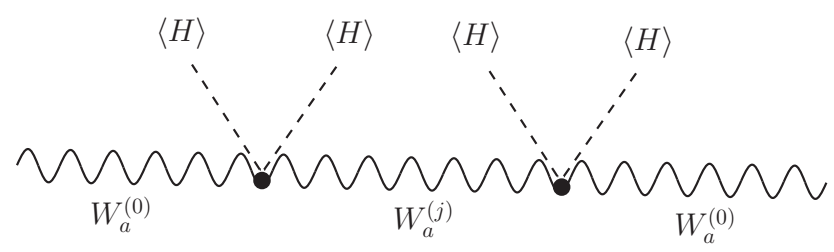

Figure 3.9: Diagrams representing the exchange of the $W_{a}^{(j)}$ gauge bosons.

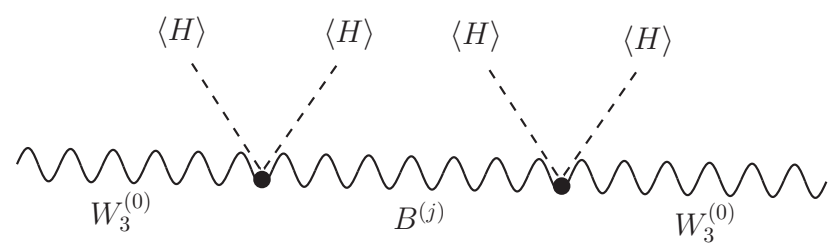

Figure 3.10: Diagrams contributing to $S$ and $T$ through the exchange of the $B^{(j)}$ gauge bosons.

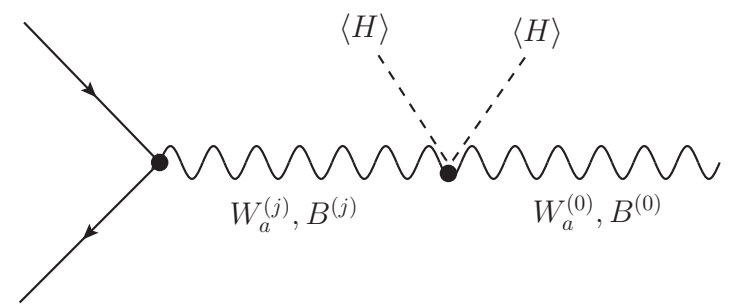

Figure 3.11: Diagrams contributing to $S$ and $T$ by vertex correction of the $W_{a}^{(0)}$ and $B^{(0)}$ gauge couplings to fermions.

The interaction Lagrangian for the gauge bosons with the Higgs localized in the $N$-th site is given by

$$
\begin{aligned}
\mathcal{L} & =\frac{1}{2}\left(D_{\mu} H\right)^{\dagger} D^{\mu} H=\frac{\tilde{g}^{2} v_{\mathrm{EW}}^{2}}{8}\left[\left(W_{1}^{N}\right)^{2}+\left(W_{2}^{N}\right)^{2}+\left(W_{3}^{N}\right)^{2}\right] \\
& +\frac{\tilde{g} \tilde{g}^{\prime} v_{\mathrm{EW}}^{2}}{4}\left(W_{3}^{N} B^{N}\right)+\frac{\tilde{g}^{\prime 2} v_{\mathrm{EW}}^{2}}{8}\left(B^{N}\right)^{2}
\end{aligned}
$$

where the Lorentz indices are omitted, and we use the usual Higgs vev $\langle H\rangle^{t}=$ $\left(0, v_{E M} / \sqrt{2}\right)$ and the covariant derivative $D^{\mu}=\partial^{\mu}+i \tilde{g} T^{a} W_{a}^{\mu, N}+i \tilde{g}^{\prime} Y B^{\mu, N}$. Ex- 
panding in Lagrangian (3.30) the fields $W_{\mu, a}^{N}$ in the mass eigenstate basis as

$$
W_{\mu, a}^{N}=\sum_{j=0}^{N} f_{N, j} W_{\mu, a}^{(j)}
$$

and analogously for $B_{\mu}^{N}$, we see that the Higgs vev insertions induce mixings between the gauge boson modes. These mixing effects lead to contributions to $S$ and $T$ parameters at tree level. The mixings between the zero mode gauge bosons and their excited states are shown in Figs. 3.9 and 3.10. Notice that in (3.30) the $W_{\mu}^{ \pm}$ zero modes have mixings only with their own modes, whereas the $W_{\mu}^{3}$ and $B_{\mu}$ zero modes mix with the massive modes of each other. The vertex for the $W W$ mixing (3.9) is given by

$$
\frac{i(N+1) g^{2} v_{\mathrm{EW}}^{2}}{4} f_{N, 0} f_{N, j}
$$

and for the $W B$ mixing (3.10) it is

$$
\frac{i(N+1) g g^{\prime} v_{\mathrm{EW}}^{2}}{4} f_{N, 0} f_{N, j}
$$

In both cases we use the expression (3.22) to relate the $\tilde{g}^{\left({ }^{\prime}\right)}$ with the Standard Model gauge couplings. Using these mixing vertices, and the corresponding first massive mode propagator valued at $q^{2}=0$, we obtain the vacuum polarizations functions

$$
\begin{aligned}
& i g^{2} \Pi_{11}(0)=-i g^{2}\left[\frac{(N+1) v_{\mathrm{EW}}^{2}}{4} f_{N, 0} f_{N, 1}\right]^{2}\left(\frac{g}{M_{1}}\right)^{2}, \\
& i g^{2} \Pi_{33}(0)=-i g^{2}\left[\frac{(N+1) v_{\mathrm{EW}}^{2}}{4} f_{N, 0} f_{N, 1}\right]^{2}\left[\left(\frac{g}{M_{1}}\right)^{2}+\left(\frac{g^{\prime}}{M_{1}}\right)^{2}\right]
\end{aligned}
$$

where the $M_{1}$ is the mass of the corresponding first massive mode. Note that we are only considering the contribution of the first excited state. This is justified since this model has a coarse discretization, that is, we are using just five sites $(N=4)$ to generate a large hierarchy of scales in the quiver. For instance, from the link fields parametrization $f_{j}=f q^{j}(2.5)$, we see that the mass of the lightest massive mode, which is of the order of the last link field vev, is $f_{4}=f q^{4} \sim \mathcal{O}(1) \mathrm{TeV}$. The next massive state has mass of order $f_{3}=f q^{3} \sim 10^{4} \mathrm{TeV}$. Therefore, we can safely 
ignore the contributions of the next states. These exchange diagrams generate the following contribution to the $T$ parameter (3.29)

$$
\begin{aligned}
T_{e}=\frac{16 \pi}{v_{\mathrm{EW}}^{2} e^{2}}\left[\Pi_{11}(0)-\Pi_{33}(0)\right] & =\frac{g^{\prime 2} \pi}{e^{2}}\left[(N+1) f_{N, 0} f_{N, 1}\right]^{2}\left(\frac{v_{\mathrm{EW}}}{M_{1}}\right)^{2} \\
& \simeq 0.05 \times\left(\frac{3 \mathrm{TeV}}{M_{1}}\right)^{2} .
\end{aligned}
$$

The corresponding contribution to the $S$ parameter (3.28) depends on $\Pi_{i i}^{\prime}(0) \propto$ $\left(\frac{d}{d q^{2}} \frac{1}{q^{2}-M_{1}^{2}}\right)_{q^{2}=0}=-\frac{1}{M_{1}^{4}}$, so it is suppressed by a factor of $v_{\mathrm{EW}}^{4} / M_{1}^{4}$ and is not the leading source of $S$. On the other hand, as we will see, the $S$ parameter receives much larger contributions from the universal shifts of gauge couplings that result from diagrams like the one in Fig. 3.11.

If we absorbed the universal part of the vertex corrections coming from diagrams like the one in Fig. 3.11 to a redefinition of the electroweak gauge fields, this will restore the gauge couplings to their Standard Model values, but will generate contributions to the $S$ and $T$ parameters similarly to what occurs in Randall-Sundrum models [115]. These fields redefinitions are given by

$$
\begin{aligned}
W^{ \pm} & \rightarrow W^{ \pm}\left(1-g^{2} \delta\right) \\
W^{3} & \rightarrow W^{3}\left(1-g^{2} \delta\right)+B g g^{\prime} \delta \\
B & \rightarrow B\left(1-g^{\prime 2} \delta\right)+W^{3} g g^{\prime} \delta,
\end{aligned}
$$

with

$$
\delta=-\left(\frac{v_{\mathrm{EW}}}{M_{1}}\right)^{2} f_{N, 0} f_{N, 1} \frac{(N+1)^{2}}{4} \frac{g_{01}}{g},
$$

where $g_{01}$ is the coupling of the zero mode fermions to the first excited state of a gauge boson, given by the expressions (3.24) and (3.25). These redefinitions generate the following contributions to $S$ and $T$

$$
\begin{aligned}
S_{v} & =32 \pi \delta \\
T_{v} & =\frac{8 \pi}{\cos ^{2} \theta_{W}} \delta .
\end{aligned}
$$

As we had anticipated, case A, which minimizes flavor violation, results in larger 
contributions to the oblique parameters. This happens because when the left-handed zero mode fermions are localized close to the $N$-th site (IR), there is an increase in gauge couplings universal shifts induced by diagrams like the one in Fig. 3.11. In Figs. 3.3-3.8 we see that for fermions localized in the UV plateau $\left.\frac{g_{01}^{L}}{g}\right|_{U V} \simeq-0.5$, and for those localized in the IR plateau $\left.\frac{g_{01}^{L}}{g}\right|_{I R} \simeq 2.0$. Therefore, comparing case A with case $\mathbf{B}$, where the left-handed fermions are localized towards the zeroth site (UV), the vertex contributions to $S$ and $T$ are about four times larger in case $\mathbf{A}$ than the ones in case $\mathbf{B}$. The results for case $\mathbf{B}$, adding the exchange and vertex contributions, are given by

$$
\begin{aligned}
& S \simeq 0.17 \times\left(\frac{3 \mathrm{TeV}}{M_{1}}\right)^{2}, \\
& T \simeq 0.16 \times\left(\frac{3 \mathrm{TeV}}{M_{1}}\right)^{2} .
\end{aligned}
$$

These results must be compared with the experimental fit to the oblique parameters, i.e., $S^{\exp }=0.03 \pm 0.10$ and $T^{\exp }=0.05 \pm 0.12$ with $m_{t}=173 \mathrm{GeV}$ and $m_{h}=$ $126 \mathrm{GeV}$ as reference values [116]. Thus, we conclude that a mass scale of about $M_{1} \simeq 3 \mathrm{TeV}$ is in agreement within the $95 \%$ C.L. bounds. Therefore, the results present here together with the bounds in Subsec. 3.3.1 show that this mass scale is enough to pass all flavor and electroweak constraints. 


\subsection{Resonances from Quiver Theories}

In the previous sections we have shown that full-hierarchy quiver theories with few sites differ significantly from warped extra dimension models. Therefore, a detailed phenomenological study of the former is necessary in order to search for their collider signals. We explore these signals at the LHC and derive bounds on the resonances in specific quiver models. As it was shown in Subsec.3.2, for a fixed UV cutoff, the couplings of the first excited gauge boson to the zero mode fermions depend on the number of sites. We use these couplings (given by (3.24) and (3.25)) for different values of $N$ to obtain the $s$-channel production of the first gauge excitation at the LHC. We consider the case when the color propagates in the quiver diagram, as well as the minimum model that consists of full-hierarchy quiver theories containing only the electroweak sector. Fig. 3.12 shows the couplings of the first excited gauge boson to the left-handed zero mode fermions for $N=4,9,15$. The dots on the black line $(N=4)$ indicate a solution for the quark sector in the interaction basis that obtains correct masses and mixing angles, the same one that we called case B in Sec. 3.3, as in Figs. 3.6-3.8.

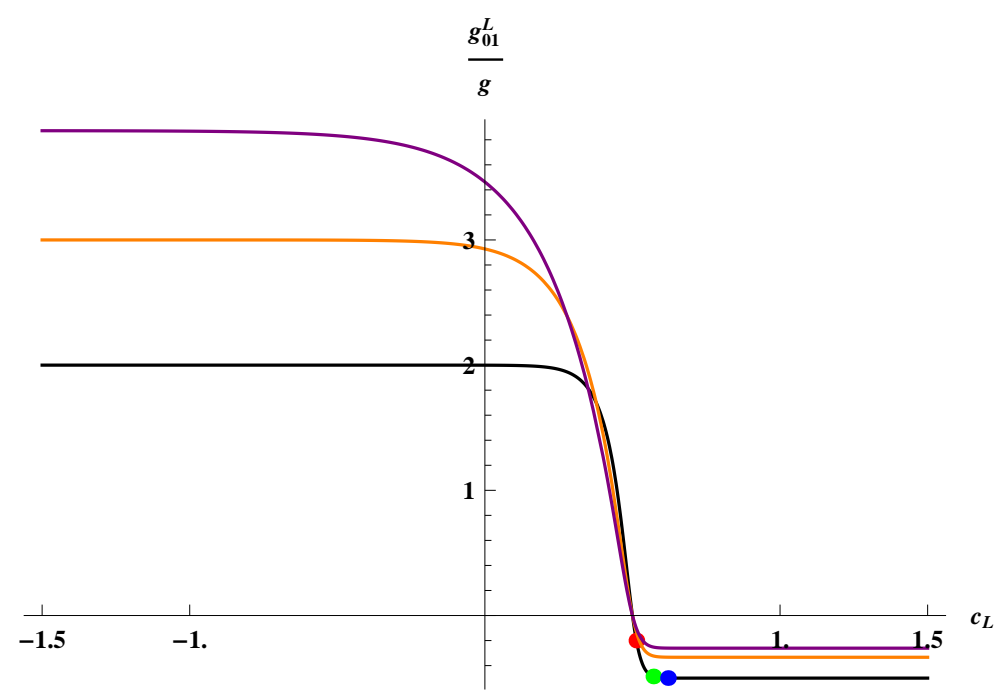

Figure 3.12: Couplings of left-handed zero mode fermions to the first massive mode of a gauge boson (normalized to the zero mode gauge boson coupling) as a function of the localization parameter $c_{L}$. For the left side of the plot and starting from the bottom: $N=4$ (black line), $N=9$ (orange line), $N=15$ (purple line). The dots on the black line show the localization of the solution called case B in Sec.3.3. 
We computed the $s$-channel production cross section at the LHC with $\sqrt{s}=8$ $\mathrm{TeV}$ and $\sqrt{s}=14 \mathrm{TeV}$ using MSTW PDFs [117]. The results with $\sqrt{s}=8 \mathrm{TeV}$ for decays of the color-octet and color-singlet excited gauge bosons to dijet, $b$-quarks, and top pairs are shown in Fig. 3.13.

As indicated in the plots (d)-(f) in Fig. 3.13, we considered the production cross section of the combination $\left(Z^{\prime}+\gamma^{\prime}\right)$ in order to obtain a conservative bound on the weakly-coupled massive gauge bosons. Thus, we are assuming that the $Z^{\prime}$ and $\gamma^{\prime}$ masses are close enough to appear degenerate at the LHC, at least in the search stages. In Fig. 3.13 we see that in general, as we increase $N$, the production cross section decreases. This happens because the absolute value of the light quarks couplings to the first gauge excitation decreases with $N$, as the light quarks are localized towards the UV region in the right side of the plot in Fig. 3.12. The only exception is plot (e), which is the decay of the color-singlet to $b$-quarks. In this case the production cross section for $N=15$ is larger than the one for $N=9$. This occurs because the left-handed coupling to the third-generation quarks for the solution with $N=9$ is very small in modulus $\left(c_{L} \simeq 0.5\right)$. This effect in this particular case exceeds the suppression factor coming from the increasing of $N$. On the other hand, we see that the production cross section for decays to $t \bar{t}$ are much more degenerate than for decays to dijet and $b \bar{b}$. This is related to the fact that the values of the couplings in the IR region increase with $N$, see left side of the plot in Fig. 3.12. As the right-handed top is localized towards the IR to generate the large top mass, the right-handed top coupling grows with $N$, which partially compensates the decrease of the light quarks couplings. The bounds on resonances mass in full-hierarchy quiver theories are shown in Tabs. 3.4 and 3.5. These constraints were extracted from the LHC data with $\sqrt{s}=8 \mathrm{TeV}$ [118-120]. The dashes in Tabs. 3.4 and 3.5 indicate the cases where there are no bounds, that is, the resonance production cross section is lower than the current LHC constraints, and additionally, these bounds are too low to be consistent with flavor and electroweak limits. The $b \bar{b}$ production cross section only gives a bound for the color-octet excited state when $N=4$, which is $M_{G}>1.6$ $\mathrm{TeV}$. For the other cases there is no bound coming from the $b$-quarks production cross sections. These results show that the most stringent direct bound comes from dijet on the color-octet excitation for the case $N=4$, which is $M_{G}>3 \mathrm{TeV}$. 


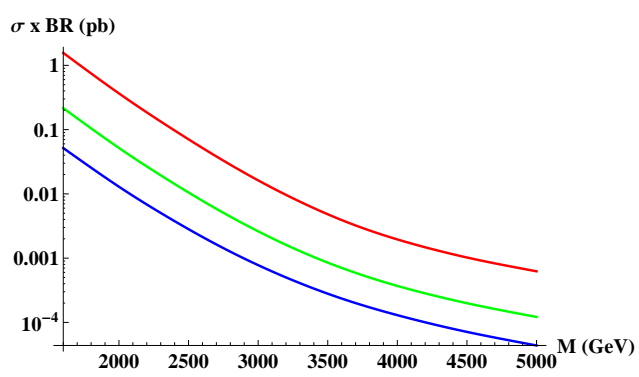

(a) : $G^{a} \rightarrow j j$

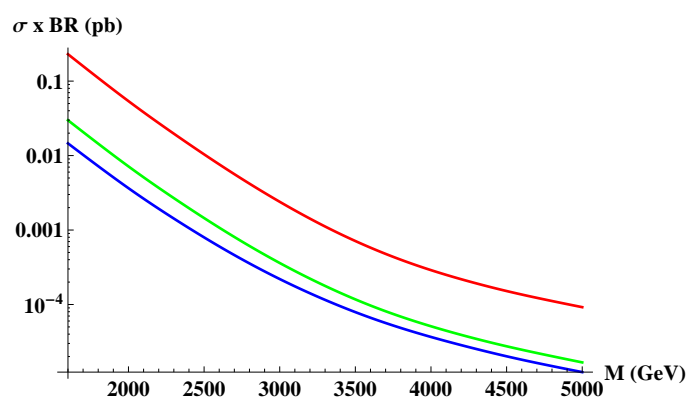

(b) : $G^{a} \rightarrow b \bar{b}$

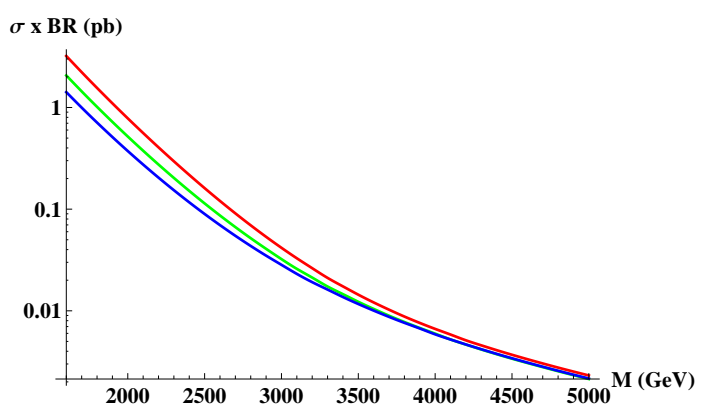

(c): $G^{a} \rightarrow t \bar{t}$

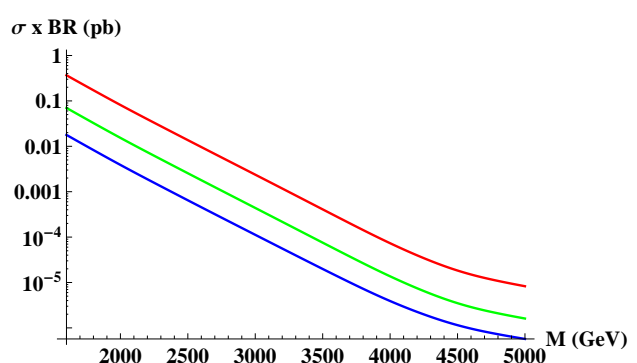

$(\mathbf{d}):\left(Z^{\prime}+\gamma^{\prime}\right) \rightarrow j j$

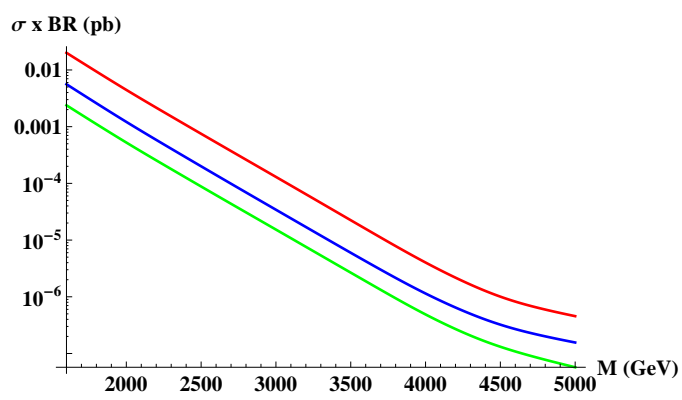

(e): $\left(Z^{\prime}+\gamma^{\prime}\right) \rightarrow b \bar{b}$

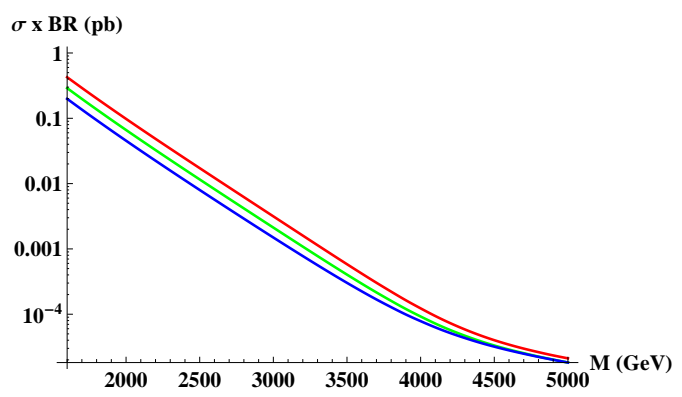

$(\mathbf{f}):\left(Z^{\prime}+\gamma^{\prime}\right) \rightarrow t \bar{t}$

Figure 3.13: Production cross sections at the LHC with $\sqrt{s}=8 \mathrm{TeV}$ as a function of the resonance mass for $N=4$ (red), $N=9$ (green), and $N=15$ (blue). On the left we have the plots for the decay of the color-octet excited gauge boson $\left(G^{a}\right)$ : to dijet (a), b-quarks (b), and top pairs (c). On the right we have the plots for the decay of the color-singlet massive gauge boson $\left(Z^{\prime}+\gamma^{\prime}\right)$ : to dijet (d), b-quarks (e), and top pairs (f).

This separation of scales between the electroweak scale $\left(v_{\mathrm{EW}} \simeq 246 \mathrm{GeV}\right)$ and the new physics scale $\left(M_{G} \gtrsim \mathcal{O}(1) \mathrm{TeV}\right)$ is naturally explained in models where the 


\begin{tabular}{cccc}
\hline \hline \multicolumn{4}{c}{ Bounds on Color-Octet } \\
\hline $\mathrm{N}$ & 4 & 9 & 15 \\
\hline$t \bar{t}[120]$ & 2.7 & 2.6 & 2.5 \\
Dijet $[118]$ & 3.0 & 1.6 & - \\
\hline \hline
\end{tabular}

Table 3.4: Direct bounds on color-octet mass in full-hierarchy quiver theories at the LHC with $\sqrt{s}=8 \mathrm{TeV}$.

\begin{tabular}{|c|c|c|c|}
\hline \multicolumn{4}{|c|}{ Bounds on Color-Singlet Mass (TeV) } \\
\hline $\mathrm{N}$ & 4 & 9 & 15 \\
\hline$t \bar{t}[120]$ & 2.1 & 2.0 & 1.8 \\
\hline Dijet [118] & 1.7 & - & - \\
\hline
\end{tabular}

Table 3.5: Direct bounds on color-singlet mass in full-hierarchy quiver theories at the LHC with $\sqrt{s}=8 \mathrm{TeV}$.

Higgs is a remnant pseudo-Nambu Goldstone Boson from the spontaneous breaking of a global symmetry $[103,104]$. The mass difference of the Higgs and the new resonances is analogous to the mass gap between the pion mass and the masses of other hadrons in QCD.

An interesting aspect of these quiver theories are their very narrow resonances even for the colored states. For instance, for the first color-octet excitation the ratios of the width to the mass are given by $\Gamma_{G} / M_{G} \simeq 4 \%(N=4), 6 \%(N=$ 9), $9 \%(N=15)$, which are very small compared with the analogous ratio for the first Kaluza-Klein gluon in Randall-Sundrum models that is $\Gamma_{K K} / M_{K K} \simeq 15 \%$ [121].

We also computed the $s$-channel production cross section at the LHC with $\sqrt{s}=14 \mathrm{TeV}$. The decays of the color-octet $\left(G^{a}\right)$ and color-singlet $\left(Z^{\prime}+\gamma^{\prime}\right)$ excited gauge bosons to dijet and top pairs in quiver theories with $N=4,15$ are shown in Figs. 3.14 and 3.15. The LHC new data can improve the constraints for this class of models and provide a better understanding of the main differences of these quiver theories with respect to other models. 


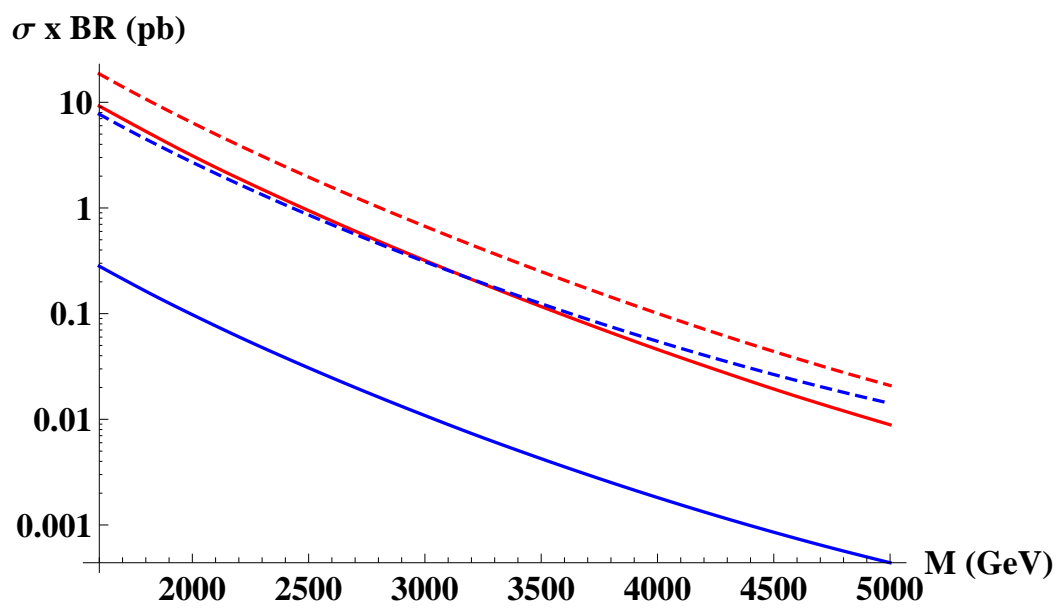

Figure 3.14: Production cross sections at the LHC with $\sqrt{s}=14 \mathrm{TeV}$ as a function of the color-octet resonance mass for $N=4$ (red) and $N=15$ (blue). The solid lines correspond to the decay of the color-octet excited gauge boson to dijet, and the dashed lines to top pairs.

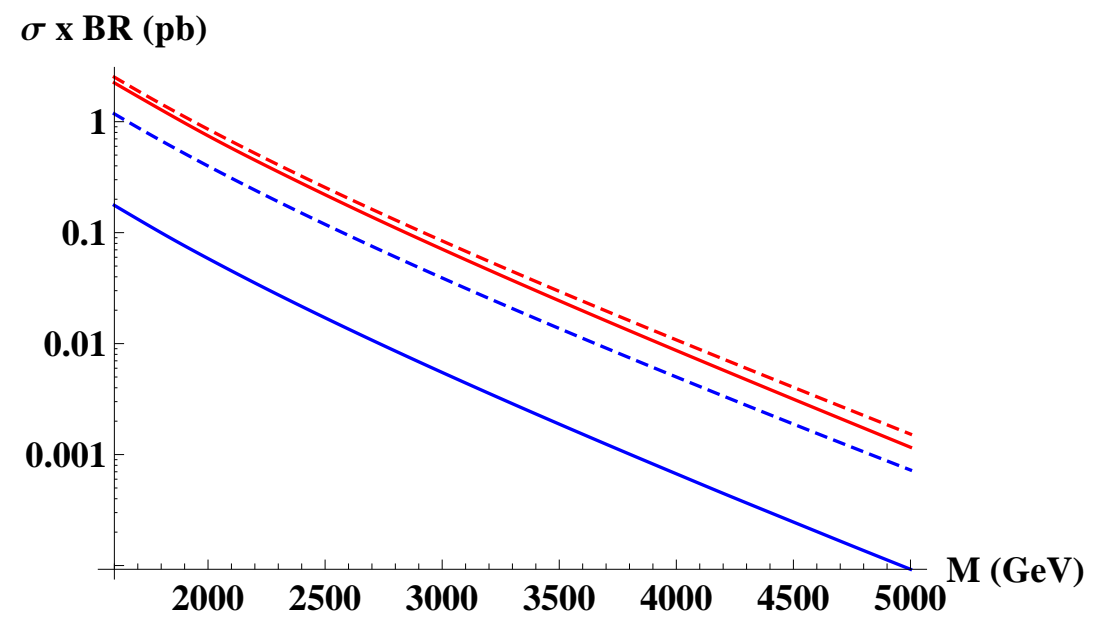

Figure 3.15: Production cross sections at the LHC with $\sqrt{s}=14 \mathrm{TeV}$ as a function of the color-singlet resonance mass for $N=4$ (red) and $N=15$ (blue). The solid lines correspond to the decay of the color-singlet excited gauge boson to dijet, and the dashed lines to top pairs. 


\section{Chapter 4}

\section{Realizing the Relaxion with $\mathrm{N}$-site Models}

\subsection{The Relaxion Idea}

The sensitivity of the Higgs mass to UV physics has been one of the leading motivations for searches of beyond SM physics at the LHC; however, until now, there is no compelling evidence of new physics at the LHC data. A new option to explain the smallness of the Higgs mass is provided by the relaxion models, which explain the radiative stability of the Higgs sector through the cosmological relaxation mechanism of the electroweak scale [76]. In these scenarios we have a scalar field starting at some large value and slowly decreasing during the inflationary epoch $[122,123]$. As an illustration, consider the relaxion model [76,77]:

$$
V(\phi, H)=\Lambda^{3} g \phi-\frac{1}{2} \Lambda^{2}\left(1-\frac{g \phi}{\Lambda}\right) H^{2}+\Lambda_{c}^{4}(H) \cos (\phi / f)+\cdots,
$$

where $H$ is the Higgs field, $\Lambda$ is the cutoff of the model, $\phi$ is the relaxion field (assumed to be a pseudo-Nambu-Goldstone Boson (pNGB) with decay constant $f$ ), the spurion $g$ quantifies the explicit breaking of the discrete shift symmetry and $\Lambda_{c}(H)$ is a scale depending on the Higgs vev so that $\Lambda_{c}(H) \neq 0 \leftrightarrow\langle H\rangle \neq 0$.

It is 'technically natural' ${ }^{1}$ to set $g$ to small values, so the first term in Eq.4.1 is responsible for the slow roll of $\phi$. Once the coefficient of $H^{2}$ on the second term

\footnotetext{
${ }^{1}$ See Chapter 1 for a discussion about naturalness.
} 
becomes negative $H$ acquires a vev and one can see that for $\Lambda \approx g \phi$ the Higgs mass is much smaller than $\Lambda$. As $\Lambda_{c}(H) \neq 0, \phi$ gets trapped close to this phase transition (which fixes $\langle H\rangle$ ). If this is to work in a natural way we must assume $\phi$ scanned the typical range of field values $\Delta \phi \sim \Lambda / g \gg \Lambda$.

There are relevant concerns regarding this idea:

1. Although having field excursions larger than the cutoff of the effective theory is not a problem in itself, it might be problematic to construct a theory that could consistently generate these large excursions, especially if the UV theory includes quantum gravity [124-127].

2. Other essential feature of Eq. 4.1 is the presence of a linear term that explicitly breaks a gauge symmetry (the axion shift symmetry), which is inconsistent with the pNGB nature of the relaxion [128].

This second point can be avoided if all operators involving $\phi$ are periodic, but with very different periods, and the linear term is nothing but a small region in an oscillation of longer period. A simple way to generate such oscillations is to produce a large hierarchy between the decay constants [129-138]:

$$
V(\phi, H) \sim \Lambda^{4} \cos \left(\frac{\phi}{F}\right)+\Lambda_{c}^{4}(H) \cos \left(\frac{\phi}{f}\right)
$$

where $F \gg f$. If additionally $F>\Lambda$ then the first point is also addressed, because $\phi$ will have a compact field space of size $2 \pi F$ (we will comment on gravity related problems below).

An explicit example is proposed in [129] to generate an effective super-Planckian field range, by considering $N+1$ complex scalars with the same decay constant $f<M_{\mathrm{Pl}}$. By adding a conveniently chosen breaking term, the global $U(1)^{N+1}$ is explicitly broken to $U(1)$ and the remaining pNGB has a decay constant which exponentially depends on the number of fields as $F \gg e^{c N} f$, where $c \sim \mathcal{O}(1)$. It is emphasized in [129] that this construction cannot be interpreted as a deconstructed extra dimension, i.e. there is no continuum limit for this model. (see [139-141] for other approaches achieving similar results).

A relevant concern arising when attempting to include gravity in the UV theory is the so-called weak gravity conjecture [124], which limits how small the coupling 
constants in gauge theories may be. Although there is no rigorous proof attesting its validity, the conjecture and its versions $[126,127]$ seem to satisfy all tests proposed so far. Assuming an unbroken $U(1)$ gauge symmetry in the low-energy theory, the conjecture is necessary to avoid a spectrum containing a large number of charged black hole remnants. The black holes' stability is guaranteed if there is no particle to which the remnants can decay and radiate away all their charge. Once one assumes negligible kinetic mixing among the gauge fields and minimal charge assignments, the conjecture can be directly applied to multiple $U(1)$ gauge interactions, resulting in an even stronger constraint than in the single $U(1)$ case $[125,126]$. In a nonabelian setup, the conjecture is not yet sufficiently explored, however, it is expected that the usual arguments will also apply to the non-abelian case [142-145].

In the following we present a different approach that can deal with the issues discussed previously and at the same time indicates a different strategy to search for UV completions for the relaxation mechanism. The two main advantages of our approach are that: (i) the model does have a continuum limit that could be interpreted as an extra dimension; and (ii) we show that the desired features can be obtained from non-abelian groups, allowing for controlled (asymptotically free) UV behaviour.

\subsection{Minimal Model}

Let us consider a $2 N$-site model represented in Fig. 4.1, where each site represents a global symmetry group ${ }^{2}, S U(2)$ (the construction is trivially generalized for other groups).

\footnotetext{
${ }^{2}$ It is well known that in a theory of quantum gravity, all global symmetries are violated (see e.g. [146]). For this reason, the model we propose in Eq. 4.3 cannot be regarded as a consistent description for arbitrary energy scales. However, it may be seen as an effective few site description of an extra dimension (see Sec.2.2). In this case, the global symmetries are gauged, and this concern disappears.
} 


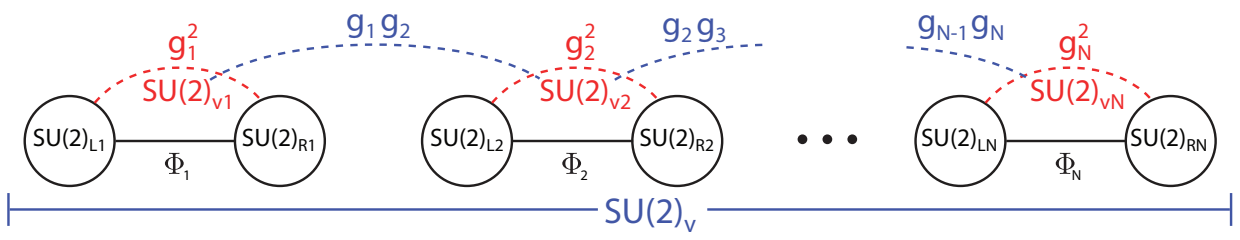

Figure 4.1: Diagram for a $2 N$-site model. The symmetry groups and link fields are in black. In red (blue) we show the effect of the $g_{j}^{2}\left(g_{j} g_{j+1}\right)$ explicit breakings, and the resulting preserved groups.

The Lagrangian for the link fields reads:

$$
\begin{aligned}
\mathcal{L}_{\Phi} & =\sum_{j=1}^{N} \operatorname{Tr}\left[\partial_{\mu} \Phi_{j}^{\dagger} \partial^{\mu} \Phi_{j}+\frac{f^{3}}{2}\left(2-\delta_{j, 1}-\delta_{j, N}\right) g_{j}^{2}\left(\Phi_{j}+\Phi_{j}^{\dagger}\right)\right] \\
& -\frac{f^{2}}{2} \sum_{j=1}^{N-1} g_{j} g_{j+1} \operatorname{Tr}\left[\left(\Phi_{j}-\Phi_{j}^{\dagger}\right)\left(\Phi_{j+1}-\Phi_{j+1}^{\dagger}\right)\right],
\end{aligned}
$$

where the $\Phi_{j}$ are scalars transforming as $\Phi_{j} \rightarrow L_{j} \Phi_{j} R_{j}^{\dagger}$, under adjacent $S U(2)$ groups. We assume the $\Phi_{j}$ acquire a vev $\left\langle\Phi_{j}\right\rangle \equiv f / 2$, spontaneously breaking $S U(2)_{L_{j}} \times S U(2)_{R_{j}} \rightarrow S U(2)_{V_{j}}$. In the low energy limit, these fields are non-linearly realized as:

$$
\Phi_{j} \rightarrow \frac{f}{2} e^{i \vec{\pi}_{j} \cdot \vec{\sigma} / f}=\frac{f}{2} \cos \left(\frac{\pi_{j}}{f}\right)+i \frac{f}{2} \frac{\vec{\pi}_{j} \cdot \vec{\sigma}}{\pi_{j}} \sin \left(\frac{\pi_{j}}{f}\right)
$$

where $\vec{\sigma}$ are the Pauli matrices, $\vec{\pi}_{j}$ are the NGB multiplets and $\pi_{j} \equiv \sqrt{\vec{\pi}_{j} \cdot \vec{\pi}_{j}}$.

The Lagrangian contains terms that explicitly break some global symmetries. These parameters are assumed to be small spurions generated at a higher scale and may be chosen such that they give a mass to all but one linear combination of the $\vec{\pi}_{j}$. The terms with $g_{j}$ explicitly break the chiral symmetries to the vector combination, $S U(2)_{L_{j}} \times S U(2)_{R_{j}} \rightarrow S U(2)_{V_{j}}$, while the terms with $g_{j} g_{j+1}$ break $S U(2)_{V_{j}} \times S U(2)_{V_{j+1}} \rightarrow S U(2)_{V_{j, j+1}}$. Taken together these terms break explicitly all symmetries down to a diagonal $S U(2)_{V}$. However, due to the peculiar structure of the breaking parameters, one combination of the $\vec{\pi}_{j}$ remains accidentally lighter, gaining a small mass only at higher order. Additional breaking terms (involving three or more powers of the $\Phi_{j}$ fields) could be present, but we will assume that they are suppressed in relation to those in Eq. 4.3. 
The Lagrangian in terms of the Goldstone fields is:

$$
\begin{aligned}
\mathcal{L}_{\pi} & =\sum_{j=1}^{N}\left[\frac{1}{2} \partial_{\mu} \vec{\pi}_{j} \cdot \partial^{\mu} \vec{\pi}_{j}+f^{4}\left(2-\delta_{j, 1}-\delta_{j, N}\right) g_{j}^{2} \cos \left(\frac{\pi_{j}}{f}\right)\right] \\
& +f^{4} \sum_{j=1}^{N-1} g_{j} g_{j+1} \frac{\vec{\pi}_{j} \cdot \vec{\pi}_{j+1}}{\pi_{j} \pi_{j+1}} \sin \left(\frac{\pi_{j}}{f}\right) \sin \left(\frac{\pi_{j+1}}{f}\right),
\end{aligned}
$$

where we omitted terms corresponding to interactions with two derivatives. Expanding to quadratic order, we obtain the mass matrix for the $\vec{\pi}_{j}$, which is independent of the $S U(2)$ index:

$$
\vec{\pi}^{t} \cdot M_{\pi}^{2} \cdot \vec{\pi} \equiv \sum_{j=1}^{N-1} f^{2}\left(g_{j} \vec{\pi}_{j}-g_{j+1} \vec{\pi}_{j+1}\right)^{2}
$$

where $\vec{\pi}^{t} \equiv\left(\vec{\pi}_{1}, \cdots, \vec{\pi}_{N}\right)$. The parametrization $g_{j} \rightarrow q^{j}$, with $0<q<1$, results in a mass matrix for the pNGBs that is identical to the one obtained for a pNGB Wilson line (zero mode) in the deconstruction of $\mathrm{AdS}_{5}$ (Eq. 2.31) as obtained in the Sec. $2.3[93,94]$ :

$$
M_{\pi}^{2}=f^{2}\left(\begin{array}{cccccc}
q^{2} & -q^{3} & 0 & \ldots & 0 & 0 \\
-q^{3} & 2 q^{4} & -q^{5} & \ldots & 0 & 0 \\
0 & -q^{5} & 2 q^{6} & \ldots & 0 & 0 \\
\vdots & \vdots & \vdots & \ldots & \vdots & \vdots \\
0 & 0 & 0 & \ldots & 2 q^{2(N-1)} & -q^{2 N-1} \\
0 & 0 & 0 & \ldots & -q^{2 N-1} & q^{2 N}
\end{array}\right)
$$

As $\operatorname{det}\left[M_{\pi}^{2}\right]=0$, this matrix has a zero mode (at tree level), as emphasized previously. Its profile is given by (see Eq. 2.35):

$$
\vec{\eta}_{0}=\sum_{j=1}^{N} \frac{q^{N-j}}{\sqrt{\sum_{k=1}^{N} q^{2(k-1)}}} \vec{\pi}_{j},
$$

which is similar to the result found in [129]. One sees that $\vec{\eta}_{0}$ is exponentially localized at the last site. It is important to note that, in contrast with [129], since 
$q<1$ our matrix does admit a continuum limit.

Since $\vec{\eta}_{0}$ has a mass much smaller than the other states ${ }^{3}$, one is justified to consider it as the relaxion field, since the other modes rapidly lose coherence on scales larger than their Compton wavelength and may thus be assumed to be constant on the scale $m_{\eta_{0}}^{-1}$. They correspond to immaterial phase shifts in the potential of $\vec{\eta}_{0}$. In terms of $\vec{\eta}_{0}$, one obtains the following Lagrangian after integrating out the other pNGBs:

$$
\begin{aligned}
\mathcal{L}_{\eta} & =\sum_{j=1}^{N}\left[\frac{1}{2} \partial_{\mu} \vec{\eta}_{0} \cdot \partial^{\mu} \vec{\eta}_{0}+f^{4}\left(2-\delta_{j, 1}-\delta_{j, N}\right) q^{2 j} \cos \frac{\eta_{0}}{f_{j}}\right] \\
& +\sum_{j=1}^{N-1} f^{4} q^{2 j+1} \sin \frac{\eta_{0}}{f_{j}} \sin \frac{\eta_{0}}{f_{j+1}},
\end{aligned}
$$

where $\eta_{0} \equiv \sqrt{\vec{\eta}_{0} \cdot \vec{\eta}_{0}}$ and the effective decay constants are given by:

$$
f_{j} \equiv f \frac{\sqrt{\sum_{k=1}^{N} q^{2(k-1)}}}{q^{N-j}} \equiv f q^{j-N} \mathcal{C}_{N}
$$

where $\mathcal{C}_{N} \equiv \sqrt{\frac{q^{2 N}-1}{q^{2}-1}}$. One sees that a large hierarchy of decay constants is generated, from the largest $f_{\max }=f_{1} \approx f / q^{N-1}$ to the smallest $f_{\min }=f_{N} \approx f$, as we wanted.

Regarding the radiative stability of the potential, we find that interactions with $p$ external $\vec{\eta}_{0}$ legs scale as $c_{p} \sim q^{2 N} f^{4-p}$ and renormalize multiplicatively (as expected, since all the couplings in the Lagrangian Eq. 4.9 are spurions), so the whole potential is radiatively stable up to small corrections.

\footnotetext{
${ }^{3}$ At tree level, for $q \ll 1$, the spectrum is approximately given by $m_{j}^{2} \approx f^{2} q^{2(j-1)}$ for $1<j \leq N$ plus a zero mode. Expanding Eq. 4.9, a quartic term is generated of order $q^{2 N}\left(\vec{\eta}_{0} \cdot \vec{\eta}_{0}\right)^{2}$. Closing the loop, one obtains a mass for $\vec{\eta}_{0}$ of order $m_{\eta_{0}}^{2}=f^{2} q^{2 N}$, which is a factor of $q^{2}$ smaller than the lightest tree level mass, hence the approximation scheme is consistent.
} 


\subsection{Higgs-Axion Interplay}

If the lightest $\mathrm{pNGB}$ is to function as a relaxion, its potential must be such that no local minima stops it when the Higgs vev is zero. The potential in Eq. 4.9 is dominated by the oscillation with the largest amplitude and period, $-f^{4} q^{2} \cos \frac{\eta_{0}}{f_{1}}$, which grows monotonically in $0<\eta_{0}<\pi f_{1}$ (which will be our region of interest). To check that the other oscillations do not get the field stuck we need to consider:

$$
\begin{aligned}
& \frac{\partial V_{\eta}}{\partial \eta_{0}}=\frac{f^{3} q^{N}}{\mathcal{C}_{N}} \sum_{j=1}^{N} q^{j} \sin \left(\frac{\eta_{0}}{f_{j}}\right)\left\{\left(2-\delta_{j, 1}-\delta_{j, N}\right)+\right. \\
& \left.-\left(1-\delta_{j, 1}\right) \cos \left(\frac{\eta_{0}}{f_{j-1}}\right)-\left(1-\delta_{j, N}\right) \cos \left(\frac{\eta_{0}}{f_{j+1}}\right)\right\} .
\end{aligned}
$$

The constant $\frac{f^{3} q^{N}}{\mathcal{C}_{N}}$ is positive for any $q<1$ and $N>1$, and the term between braces is bounded between 0 and 4 . The leading term for small $q$ is:

$$
\frac{f^{3} q^{N}}{\mathcal{C}_{N}} q \sin \left(\frac{\eta_{0}}{f_{1}}\right)\left\{1-\cos \left(\frac{\eta_{0}}{f_{2}}\right)\right\},
$$

which is never negative for $0<\eta_{0}<\pi f_{1}$ and is only zero at $\eta_{0}^{m} \equiv 2 \pi m q f_{1}$, with $m=\{0,1,2 \ldots\}$. Close to these points the sign of the derivative will come from terms with higher powers of $q$. The one multiplying $q^{N+2}$ is:

$$
\sin \left(\frac{\eta_{0}^{m}}{f_{2}}\right) \approx \frac{\eta_{0}}{q f_{1}}-2 \pi m
$$

This sine will push the derivative to negative values near $\eta_{0}^{m}$, generating shallow minima (similar arguments apply to the next terms in the $q$-expansion). The derivative only remains negative while the term in Eq. 4.12 is smaller than the $\mathcal{O}\left(q^{N+2}\right)$ term, so these minima become less and less important as $q$ gets smaller. In fact, the height of the barrier between two adjacent minima decreases as $q^{4}$, the width decreases as $q^{2-N} \mathcal{C}_{N}$ and we expect the field to be able to proceed rolling down for the typical values of $q$ considered below. The shape of the potential with decreasing $q$ can be seen in Figure 4.2. One can see that, despite the use of quite large values of $q$ and a scaling factor $\alpha$ to exaggerate the features of the potential, the slope quickly gets smooth. 


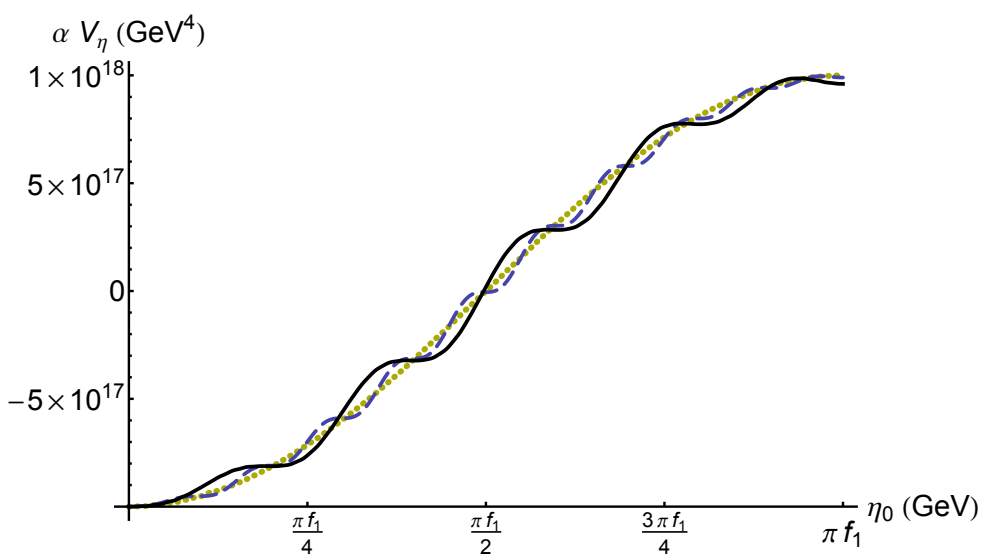

Figure 4.2: Potential $V_{\eta}\left(\eta_{0}\right)$ for different values of $q$ and $N=3$. A factor $\alpha$ was introduced to allow easy comparison between the curves. The black, blue (dashed) and yellow (dotted) curves have respectively $(q=0.1, \alpha=1),\left(q=0.05, \alpha=10^{3}\right)$ and $\left(q=0.01, \alpha=10^{10}\right)$. Note that these values of $q$ are much larger than the realistic ones, in order to exacerbate the features in the potential.

We include the Higgs by multiplying the Lagrangian by $1+|H|^{2} / \Lambda^{2}$, where $\Lambda \approx 4 \pi f$. Adding in the Higgs potential and kinetic term, the full Lagrangian is now:

$$
\mathcal{L}_{\eta, H}=\left(1+\frac{|H|^{2}}{\Lambda^{2}}\right) \mathcal{L}_{\eta}+\left|D_{\mu} H\right|^{2}+\frac{\Lambda^{2}}{2}|H|^{2}-\frac{\lambda_{H}}{4}|H|^{4} .
$$

Once the Higgs is set to its vev, $\langle h\rangle=v_{\mathrm{EW}}>0$, the slope equation is the same as Eq. 4.11 , multiplied by $\left(1+v_{\mathrm{EW}}^{2} /\left(2 \Lambda^{2}\right)\right)$. The field $\phi$ should stop rolling when this expression is approximately zero. However, this clearly has no solutions apart from the trivial one $v_{\mathrm{EW}}^{2}=-2 \Lambda^{2}$, which is undesirable.

With the current Lagrangian, having $v_{\text {EW }} \ll f$ is untenable. In order to fix this, we add the following breaking term at the last site, which would be equivalent, in the continuum limit, to a deformation of the metric in the infrared (see again Sec. 2.2):

$$
\mathcal{L}_{\eta, H} \rightarrow \mathcal{L}_{\eta, H}+\epsilon \frac{\Lambda_{c}}{16 \pi} \operatorname{Tr}\left[\Phi_{N}+\Phi_{N}^{\dagger}\right]|H|^{2}
$$

where $\epsilon$ is a small parameter, and $\Lambda_{c}$ is a new scale, which we could assume is generated at lower energies to avoid spoiling the results of the previous section (see Eq. 4.2). However, as pointed out in [77], a small $\Lambda_{c}$ scale leads to a coincidence problem (i.e., $\Lambda \gg \Lambda_{c} \sim \mathrm{TeV}$ ) for the model. We will then take $\Lambda_{c} \approx \Lambda \approx 4 \pi f$ and 
discuss below how to avoid the problems generated by this choice.

Once this operator is added, the relaxion potential acquires the term

$$
\epsilon f^{2}|H|^{2} \cos \frac{\eta_{0}}{f_{N}}
$$

giving the relaxion a small mass. By closing the loop of $H, \epsilon f^{4} \cos \left(\eta_{0} / f_{N}\right)$ is generated, which can spoil the relaxation mechanism. One possible solution is to adopt the double scanner mechanism of [77], that is, we may add a scalar singlet to control the amplitude of the additional term. As emphasized in [77], the new field needs an even larger field excursion than the relaxion. This can be accommodated in our framework by replicating this scalar on the $N$-sites, provided we choose a smaller value of the $q$ parameter for this scalar. A non-trivial issue that must be addressed in a complete model is the fact that the UV completion should not couple the new scalar to the Higgs at tree level, or else one risks spoiling the relaxation [77]. For a supersymmetric version of a two-field relaxion model, see [147].

With the inclusion of (4.16), the new slope equation is given by:

$$
\begin{aligned}
& \frac{\partial V_{\eta, H}}{\partial \eta_{0}}=\frac{f^{3} q^{N+1}}{\mathcal{C}_{N}}\left\{( 1 + \frac { v _ { \mathrm { EW } } ^ { 2 } } { 2 \Lambda ^ { 2 } } ) \operatorname { s i n } ( \frac { \eta _ { 0 } } { f _ { 1 } } ) \left[1-\cos \left(\frac{\eta_{0}}{f_{2}}\right)\right.\right. \\
& \left.+\mathcal{O}(q)]-\epsilon \frac{v_{\mathrm{EW}}^{2}}{2 f^{2} q^{N+1}} \sin \left(\frac{\eta_{0}}{f_{N}}\right)\right\}+\cdots .
\end{aligned}
$$

This slope should be zero when $v_{\mathrm{EW}} \approx 246 \mathrm{GeV}$. Solving for this yields

$$
v_{\mathrm{EW}}^{2} \sim \frac{f^{2}}{\epsilon} q^{N+1} .
$$

For $q^{N+1}<\epsilon<1$, a natural electroweak scale is obtainable and $q^{N+1}$ should be identified with the relaxion coupling $g$ of [77], as in Eq.4.1.

The cutoff for our model can be estimated along the lines of [77] by considering additional constraints besides Eq. 4.18. The main bounds come from requiring that $\vec{\eta}_{0}$ does not drive inflation, i.e. $\Lambda^{2} \lesssim H_{I} M_{\mathrm{Pl}}$, where $H_{I}$ is the inflation scale and $M_{\mathrm{Pl}}$ is the reduced Planck scale, and that quantum fluctuations of $\vec{\eta}_{0}$ are less important than its classical rolling. This yields the condition that $H_{I}^{3} \lesssim q^{N+1} f^{3}$. Finally, suppressing higher order terms like $\epsilon^{2} f^{4} \cos \left(\eta_{0} / f\right)^{2}$ requires $\epsilon \lesssim v^{2} / f^{2} \sim 10^{-12}$, for 
$f=10^{8} \mathrm{GeV}$ [77]. Combining these with Eq. 4.18, we obtain:

$$
\frac{\Lambda^{6}}{f^{3} M_{\mathrm{Pl}}^{3}} \lesssim q^{N+1} \lesssim \frac{v_{\mathrm{EW}}^{4}}{f^{4}}
$$

From this, we find the upper bound of $f \lesssim 10^{8} \mathrm{GeV}$ and also that $q \lesssim 10^{-23 /(N+1)}$.

Finally, using all these constraints, we find that for $q \approx 10^{-24 /(N+1)}$ and $\epsilon \approx$ $10^{-12}$, we obtain $v_{\mathrm{EW}} \sim 10^{-6} f$ which is of the order of the electroweak scale for $f \approx 10^{8} \mathrm{GeV}$. Note that for these parameter choices, Eq. 4.18 does not depend on $N$, what is fixed is $q^{N+1}$. Of course, having a large value for $N$ allows for a larger value of $q$.

\section{Remarks}

Our model has some distinctive features when compared with previous many-field models that also address the points above $[129,130]$ :

- The $N$ fields are bi-fundamentals of $2 N$ non-abelian $S U(2)$ groups and the formalism employed can be trivially generalized to any non-abelian group. This allows for a controlled UV behavior and opens up many possibilities of model building in particle physics and inflation.

- The model has a well defined continuum limit $N \rightarrow \infty, q \rightarrow 1$, with $q^{N+1}$ kept fixed, and the mass matrix for the pNGBs in Eq. 4.7 is exactly the one obtained from a pNGB Wilson line in the deconstruction of $\mathrm{AdS}_{5}[93,94]$ (see Sec. 2.3). Even the desired relation between $v_{\mathrm{EW}}$ and $f$ (in Eq.4.18) is maintained in the continuum limit, as $f^{2} q^{N+1} \rightarrow M / g_{5}^{2} e^{-k L}$, where $L$ is the size of the extra dimension, $k$ is the curvature, $g_{5}$ is the $5 \mathrm{D}$ gauge coupling, and $M$ is the cutoff of the UV theory. In addition, we find that (up to suppressed terms) in the continuum limit (see Eq.4.10), $f_{1}=\mathcal{C}_{N} f q^{1-N} \rightarrow M /\left(g_{5} \sqrt{2 k}\right) e^{k L}$ and $f_{N}=\mathcal{C}_{N} f \rightarrow M /\left(g_{5} \sqrt{2 k}\right)$, that is $f_{1} / f_{N} \rightarrow e^{k L}$, i.e. they are related by the $\mathrm{AdS}_{5}$ warp factor. These expressions are in agreement with those obtained by [107] in $\mathrm{AdS}_{5}$. 


\section{Chapter 5}

\section{Conclusions and Remarks}

In this thesis we explored the full-hierarchy quiver theories, which is a class of $N$-site models that can generate large-scale hierarchies with just a few sites. As we discussed in the previous chapters, these four-dimensional models can be used in a broader context. We summarize in the following our main results.

- Localization in the Theory Space and Flavor Bounds: Using this class of $N$-site models we were able to obtain the quark mass hierarchy and mixings without large flavor changing neutral currents at tree level [64]. In RandallSudrum scenarios, in order to overcome the kaon physics constraints without introducing new global flavor symmetries, it is necessary to require first excited state masses of at least $M_{\mathrm{KK}} \sim 20 \mathrm{TeV}$ [59-61], generating a large gap between the first massive state and the electroweak scale. Additionally, we show that the solution we called case B in Sec. 3.3 is in agreement with the electroweak precision tests without the extension of the electroweak gauge sector to provide custodial protection, which is necessary in $\mathrm{AdS}_{5}$ models [115]. The bound on the mass of the color-octet gauge excitation for the solution case $\mathrm{B}$ is $M_{G}>3$ $\mathrm{TeV}$, which passes all flavor and electroweak precision constraints [64].

- Resonances from Quiver Theories: We also explored the phenomenology of quiver theories with different number of sites. In Sec. 3.4 we derived bounds from the LHC data on the color-octet and color-singlet excited gauge bosons from their decays to dijet, $b$-quarks and top pairs [68]. 
- $\boldsymbol{N}$-Relaxion: Inspired by the quiver theories framework, we constructed a $2 \mathrm{~N}$-site model capable of addressing two challenging points of the relaxion scenarios: the requirement for large field excursions, and a linear term that explicit breaks the axion shift symmetry. Our model generates a potential composed of many oscillatory terms with very different periods (see Eq.4.9), where the term with the larger period plays the role of the linear term in Eq.4.1. From $N$ fields acquiring expectation values of order $f$, an effective scale $f_{1}=\mathcal{C}_{N} f / q^{N-1} \gg f$ is generated and the pNGBs have a compact field space of $2 \pi f_{1}$, which allows for large field excursions. In the viable region of parameter space, we find that the cutoff of the model can be pushed up to $\Lambda \approx 4 \pi f \sim 10^{9} \mathrm{GeV}$.

We mention next some possible directions. Although the potential of the $N$-site model (Eq. 4.9) has shallow minima that do not affect the slow roll of the relaxion, adding the Higgs requires the introduction of a new term that generates large barriers for $\langle H\rangle \neq 0$. The extra breaking is proportional to a new spurion $\epsilon$ and ultimately controls the magnitude of the Higgs vev via Eq. 4.18. This operator may also spoil the relaxation mechanism via higher order corrections, but we expect these can be amended by adopting the double scanner scenario of Ref. [77]. As mentioned in Sec.4.3, this is a non-trivial issue that should be addressed in a complete model, and it would be interesting to search for a model building that can overcome this difficulty.

Furthermore, the breaking term of Eq. 4.15 is not unique, and it may be possible to avoid introducing it by considering different terms in Eq. 4.3 that automatically generate the large barriers needed to stop the rolling of the relaxion. Alternatively, one might be able to achieve the same result through changing the parametrization of the $g_{j}$ couplings in the Lagrangian in order to mimic a metric that is slightly deformed from $\mathrm{AdS}_{5}$.

A natural next step is to investigate the continuum limit of this $2 N$-site model or even change the approach by considering a model directly in the warped extra dimension, which is a promising direction to achieve an UV completion that is compatible with the weak gravity conjecture [148]. In addiction, the framework established here may find application in model building of the inflation sector, which 
also requires large field excursions. Finally, it is interesting to search for viable inflation models compatible with the relaxion proposal (see e.g. [149])

To sum up, here we explored different model building frameworks such as dimensional deconstruction, composite Higgs models, and warped extra dimension scenarios. We have combined and extended these techniques to develop theories that can generate a large hierarchy of scales. Surprisingly, by going from particle physics to cosmology (and back), we were able to apply these tools to propose an UV completion model that can solve some of the problems faced by the recently proposed cosmological relaxation mechanism of the electroweak scale.

To conclude with a personal note, we want to mention that given the high expectations regarding the next discovery in the field, the particle physics community is currently experiencing an intricate moment: from one side, the area is extremely successful, whose recent peak of excitement may be attributed to the discovery of a boson compatible with the Standard Model Higgs at the LHC in 2012. On the other hand, there is an inevitable, perhaps premature, disappointment since there is no definitive evidence of new physics at the LHC current data. This scenario should not be discouraging; instead, this is an opportune moment to focus on real challenges in fundamental physics and be inspired by many apparently end-of-road's in the past that turned out to be amazing changes of paradigm. We hope that the interchange with other areas may bring us some of the mechanisms necessary to better explore the open questions in the field. 


\section{Bibliography}

[1] B. P. Abbott et al. (LIGO Scientific Collaboration and Virgo Collaboration). Observation of Gravitational Waves from a Binary Black Hole Merger. Phys. Rev. Lett., 116:061102, 2016. 1

[2] Licia Verde. Precision cosmology, Accuracy cosmology and Statistical cosmology. Proceedings of the International Astronomical Union, 10:223-234, 2014. 1

[3] Philip Bull et al. Beyond $\Lambda$ CDM: Problems, solutions, and the road ahead. Phys. Dark Univ., 12:56-99, 2016. 1

[4] Dominik J. Schwarz, Craig J. Copi, Dragan Huterer, and Glenn D. Starkman. CMB Anomalies after Planck. 2015. [arXiv:1510.07929 [astro-ph.CO]]. 1

[5] K. A. Olive et al. Review of Particle Physics. Chin. Phys., C38:090001, 2014. http://pdg.lbl.gov/. 1, 2, 3

[6] Georges Aad et al. Observation of a new particle in the search for the Standard Model Higgs boson with the ATLAS detector at the LHC. Phys.Lett., B716:129, 2012. 2, 8

[7] Serguei Chatrchyan et al. Observation of a new boson at a mass of $125 \mathrm{GeV}$ with the CMS experiment at the LHC. Phys.Lett., B716:30-61, 2012. 2, 8

[8] ATLAS Collaboration. Higgs Public Results .

https://twiki.cern.ch/twiki/bin/view/AtlasPublic/

HiggsPublicResults. 2 
[9] CMS Collaboration. Higgs Public Results.

http://cms-results.web.cern.ch/cms-results/public-results/ publications/HIG/index.html. 2

[10] Kenneth G. Wilson. The Renormalization Group and Strong Interactions. Phys.Rev., D3:1818, 1971.

M.J.G Veltman. Second Threshold in Weak Interactions. Acta Phys.Polon, B8:475, 1977.

M.J.G Veltman. Large Higgs Mass and mu - e Universality. Phys.Lett., B70:253-254, 1977.

Leonard Susskind. Dynamics of Spontaneous Symmetry Breaking in the Weinberg-Salam Theory. Phys.Rev., D20:2619-2625, 1979. 2

[11] Gerard 't Hooft. Naturalness, chiral symmetry, and spontaneous chiral symmetry breaking. NATO Sci. Ser. B, 59:135, 1980. 2, 6

[12] Steven Weinberg. Gauge Hierarchies. Phys.Lett., B82:387, 1979. 2

[13] James D. Wells. Lectures on Higgs Boson Physics in the Standard Model and Beyond. 2009. CERN-PH-TH-2009-154 [arXiv:0909.4541 [hep-ph]]. 2, 8

[14] Frank Wilczek. The Future of particle physics as a natural science. Int.J.Mod.Phys., A13:863-886, 1998. 2

[15] Chris Quigg. Unanswered Questions in the Electroweak Theory. Ann.Rev.Nucl.Part.Sci., 59:505-555, 2009. 2

[16] Gian F. Giudice. Naturalness after LHC8. PoS, EPS-HEP2013:163, 2013. [arXiv:1307.7879 [hep-ph]]. 2

[17] G. J. Feldman, J. Hartnell, and T. Kobayashi. Long-baseline neutrino oscillation experiments. Adv. High Energy Phys., 2013:475749, 2013. 2

[18] G. Bellini, L. Ludhova, G. Ranucci, and F. L. Villante. Neutrino oscillations. Adv. High Energy Phys., 2014:191960, 2014. 2 
[19] The Royal Swedish Academy of Sciences. Neutrino oscillations. 2015. https://www.nobelprize.org/nobel_prizes/physics/laureates/ 2015/advanced-physicsprize2015.pdf. 2

[20] Francesco Vissani. Do experiments suggest a hierarchy problem? Phys. Rev., D57:7027-7030, 1998. 2

[21] Mikhail Shaposhnikov. Is there a new physics between electroweak and Planck scales? In Astroparticle Physics: Current Issues, 2007 (APCIO7) Budapest, Hungary, 2007. 2

[22] Marco Farina, Duccio Pappadopulo, and Alessandro Strumia. A modified naturalness principle and its experimental tests. JHEP, 08:022, 2013. 2

[23] C. A. Baker et al. An Improved experimental limit on the electric dipole moment of the neutron. Phys. Rev. Lett., 97:131801, 2006. 2

[24] R. D. Peccei and Helen R. Quinn. Constraints Imposed by CP Conservation in the Presence of Instantons. Phys. Rev., D16:1791-1797, 1977. 3

[25] R. D. Peccei and Helen R. Quinn. CP Conservation in the Presence of Instantons. Phys. Rev. Lett., 38:1440-1443, 1977. 3

[26] David J. E. Marsh. Axion Cosmology. Phys. Rept., 643:1-79, 2016. 3

[27] P. A. R. Ade et al. Planck 2015 results. XIII. Cosmological parameters. 2015. [arXiv:1502.01589 [astro-ph.CO]]. 3, 4

[28] E. Komatsu et al. Seven-Year Wilkinson Microwave Anisotropy Probe (WMAP) Observations: Cosmological Interpretation. Astrophys.J.Suppl., 192:18, 2011. 3

[29] Gianfranco Bertone, Dan Hooper, and Joseph Silk. Particle dark matter: Evidence, candidates and constraints. Phys.Rept., 405:279-390, 2005. 3

[30] Gianfranco Bertone, Dan Hooper, and Joseph Silk. Particle dark matter: Evidence, candidates and constraints. Phys.Rept., 405:279-390, 2005. 3 
[31] L. Bergström. Dark matter evidence, particle physics candidates and detection methods. Annalen der Physik, 524:479-496, October 2012. 3

[32] L. Baudis. Direct dark matter detection: The next decade. Physics of the Dark Universe, 1:94-108, November 2012. 3

[33] Nayara Fonseca, Renata Zukanovich Funchal, Andre Lessa, and Laura LopezHonorez. Dark Matter Constraints on Composite Higgs Models. JHEP, 1506:154, 2015. 3

[34] Nayara Fonseca, Lina Necib, and Jesse Thaler. Dark Matter, Shared Asymmetries, and Galactic Gamma Ray Signals. JCAP, 1602(02):052, 2016. 4

[35] Ryan Cooke, Max Pettini, Regina A. Jorgenson, Michael T. Murphy, and Charles C. Steidel. Precision measures of the primordial abundance of deuterium. Astrophys. J., 781(1):31, 2014. 4

[36] Tansu Daylan, Douglas P. Finkbeiner, Dan Hooper, Tim Linden, Stephen K. N. Portillo, Nicholas L. Rodd, and Tracy R. Slatyer. The characterization of the gamma-ray signal from the central Milky Way: A case for annihilating dark matter. Phys. Dark Univ., 12:1-23, 2016. 4

[37] Francesca Calore, Ilias Cholis, and Christoph Weniger. Background model systematics for the Fermi GeV excess. JCAP, 1503:038, 2015. 4

[38] Nicolás Bernal, Chee Sheng Fong, and Nayara Fonseca. Sharing but not Caring: Dark Matter and the Baryon Asymmetry of the Universe. 2016. [arXiv:1605.07188 [hep-ph]]. 4

[39] Michael E. Peskin and Daniel V. Schroeder. An Introduction to quantum field theory. Westview Press, 1995. 5, 7

[40] Matthew D. Schwartz. Quantum Field Theory and the Standard Model. Cambridge University Press, 2014. 5, 7

[41] Kenneth G. Wilson. Renormalization group and critical phenomena. 2. Phase space cell analysis of critical behavior. Phys. Rev., B4:3184-3205, 1971. 5 
[42] Kenneth G. Wilson. Renormalization group and critical phenomena. 1. Renormalization group and the Kadanoff scaling picture. Phys. Rev., B4:3174-3183, 1971. 5

[43] Kenneth G. Wilson and Michael E. Fisher. Critical exponents in 3.99 dimensions. Phys. Rev. Lett., 28:240-243, 1972. 5

[44] Fred Jegerlehner. The Standard model as a low-energy effective theory: what is triggering the Higgs mechanism? Acta Phys. Polon., B45(6):1167, 2014. 7

[45] Felix Brummer. Beyond the Standard Model . Lectures for the 2015 DESY summer student program, 2015. http://summerstudents.desy.de/hamburg/ e177730/e202992/BSM_DESY_1.pdf. 7

[46] Benjamin W. Lee, C. Quigg, and H. B. Thacker. Weak Interactions at Very High-Energies: The Role of the Higgs Boson Mass. Phys. Rev., D16:1519, 1977. 8

[47] José Ramón Espinosa. Vacuum Stability and the Higgs Boson. PoS, LATTICE2013:010, 2014. [arXiv:1311.1970 [hep-lat]]. 8

[48] Mikhail Shaposhnikov. Cosmology: theory. PoS, EPS-HEP2013:155, 2013. [arXiv:1311.4979 [hep-ph]]. 8

[49] Joan Elias-Miro, Jose R. Espinosa, Gian F. Giudice, Gino Isidori, Antonio Riotto, and Alessandro Strumia. Higgs mass implications on the stability of the electroweak vacuum. Phys. Lett., B709:222-228, 2012. 8

[50] Lisa Randall and Raman Sundrum. A Large mass hierarchy from a small extra dimension. Phys.Rev.Lett., 83:3370-3373, 1999. 9, 14

[51] Lisa Randall and Raman Sundrum. An Alternative to compactification. Phys.Rev.Lett., 83:4690-4693, 1999. 9, 14

[52] Csaba Csaki. TASI lectures on extra dimensions and branes. In From fields to strings: Circumnavigating theoretical physics. Ian Kogan memorial collection (3 volume set), pages 605-698, 2004. [,967(2004)]. 9, 18 
[53] Abdel Perez-Lorenzana. An Introduction to extra dimensions. J.Phys.Conf.Ser., 18:224-269, 2005. 9, 18

[54] Tony Gherghetta. Les Houches lectures on warped models and holography. In Particle physics beyond the standard model. Proceedings, Summer School on Theoretical Physics, 84th Session, Les Houches, France, August 1-26, 2005, pages $263-311,2006.9,18$

[55] Eduardo Ponton. TASI 2011: Four Lectures on TeV Scale Extra Dimensions. In The Dark Secrets of the Terascale, pages 283-374, 2013. 9

[56] Stephan J. Huber. Flavor violation and warped geometry. Nucl.Phys., B666:269-288, 2003. 9, 25

[57] Gustavo Burdman. Flavor violation in warped extra dimensions and CP asymmetries in B decays. Phys.Lett., B590:86-94, 2004. 9, 25

[58] Kaustubh Agashe, Gilad Perez, and Amarjit Soni. Flavor structure of warped extra dimension models. Phys.Rev., D71:016002, 2005. 9, 25

[59] Csaba Csaki, Adam Falkowski, and Andreas Weiler. The Flavor of the Composite Pseudo-Goldstone Higgs. JHEP, 0809:008, 2008. 9, 25, 40, 61

[60] Csaba Csaki, Adam Falkowski, and Andreas Weiler. A Simple Flavor Protection for RS. Phys.Rev., D80:016001, 2009. 9, 25, 40, 61

[61] Jose Santiago. Minimal Flavor Protection: A New Flavor Paradigm in Warped Models. JHEP, 0812:046, 2008. 9, 25, 40, 61

[62] Nima Arkani-Hamed, Andrew G. Cohen, and Howard Georgi. (De)constructing dimensions. Phys.Rev.Lett., 86:4757-4761, 2001. 9, 13, 20

[63] Christopher T. Hill, Stefan Pokorski, and Jing Wang. Gauge invariant effective Lagrangian for Kaluza-Klein modes. Phys.Rev., D64:105005, 2001. $9,13,20$

[64] Gustavo Burdman, Nayara Fonseca, and Leonardo de Lima. Full-hierarchy Quiver Theories of Electroweak Symmetry Breaking and Fermion Masses. JHEP, 1301:094, 2013. 9, 12, 61 
[65] Jorge de Blas, Adam Falkowski, Manuel Perez-Victoria, and Stefan Pokorski. Tools for deconstructing gauge theories in AdS(5). JHEP, 08:061, 2006. 9, 20

[66] Csaba Csaki, Adam Falkowski, and Andreas Weiler. The Flavor of the Composite Pseudo-Goldstone Higgs. JHEP, 09:008, 2008. 10

[67] Csaba Csaki, Adam Falkowski, and Andreas Weiler. A Simple Flavor Protection for RS. Phys. Rev., D80:016001, 2009. 10

[68] Gustavo Burdman, Nayara Fonseca, and Gabriela Lichtenstein. Resonances from Quiver Theories at the LHC. Phys. Rev., D88:116006, 2013. 10, 12, 61

[69] Stephen P. Martin. A Supersymmetry primer. 1997. [Adv. Ser. Direct. High Energy Phys.18,1(1998)]. 10

[70] Giuliano Panico and Andrea Wulzer. The Composite Nambu-Goldstone Higgs. Lect. Notes Phys., 913:pp.1-316, 2016. 10, 23

[71] Cédric Delaunay, Roee Ozeri, Gilad Perez, and Yotam Soreq. Probing The Atomic Higgs Force. 2016. [arXiv:1601.05087 [hep-ph]]. 10

[72] Claudia Frugiuele, Elina Fuchs, Gilad Perez, and Matthias Schlaffer. Atomic probes of new physics. 2016. [arXiv:1602.04822 [hep-ph]]. 10

[73] Daniel Bauer et al. Dark Matter in the Coming Decade: Complementary Paths to Discovery and Beyond. Phys. Dark Univ., 7-8:16-23, 2015. 10

[74] F. Ruppin, J. Billard, E. Figueroa-Feliciano, and L. Strigari. Complementarity of dark matter detectors in light of the neutrino background. Phys. Rev., D90(8):083510, 2014. 10

[75] Miguel Pato, Laura Baudis, Gianfranco Bertone, Roberto Ruiz de Austri, Louis E. Strigari, and Roberto Trotta. Complementarity of Dark Matter Direct Detection Targets. Phys. Rev., D83:083505, 2011. 10

[76] Peter W. Graham, David E. Kaplan, and Surjeet Rajendran. Cosmological Relaxation of the Electroweak Scale. Phys. Rev. Lett., 115(22):221801, 2015. 11,51 
[77] J. R. Espinosa, C. Grojean, G. Panico, A. Pomarol, O. Pujolàs, and G. Servant. Cosmological Higgs-Axion Interplay for a Naturally Small Electroweak Scale. Phys. Rev. Lett., 115(25):251803, 2015. 11, 51, 58, 59, 60, 62

[78] L. F. Abbott. A Mechanism for Reducing the Value of the Cosmological Constant. Phys. Lett., B150:427-430, 1985. 11

[79] Steven Weinberg. The Cosmological Constant Problem. Rev. Mod. Phys., 61:1-23, 1989. 11

[80] Steven Weinberg. The Cosmological constant problems. In Sources and detection of dark matter and dark energy in the universe. Proceedings, 4th International Symposium, DM 2000, Marina del Rey, USA, February 23-25, 2000, pages 18-26, 2000. 11

[81] Luis Alvarez-Gaume and Miguel A. Vazquez-Mozo. An invitation to quantum field theory. Lect. Notes Phys., 839:1-294, 2012. 11

[82] G. Hinshaw et al. Nine-Year Wilkinson Microwave Anisotropy Probe (WMAP) Observations: Cosmological Parameter Results. Astrophys. J. Suppl., 208:19, 2013. 11

[83] Gia Dvali and Alexander Vilenkin. Cosmic attractors and gauge hierarchy. Phys. Rev., D70:063501, 2004. 11

[84] Gia Dvali. Large hierarchies from attractor vacua. Phys. Rev., D74:025018, 2006. 11

[85] Paul J. Steinhardt and Neil Turok. Why the cosmological constant is small and positive. Science, 312:1180-1182, 2006. 11

[86] Nayara Fonseca, Leonardo de Lima, Camila S. Machado, and Ricardo D. Matheus. Large field excursions from a few site relaxion model. Phys. Rev., D94(1):015010, 2016. 11, 12 
[87] Jorge de Blas, Adam Falkowski, Manuel Perez-Victoria, and Stefan Pokorski. Tools for deconstructing gauge theories in AdS(5). JHEP, 0608:061, 2006. $13,17,18,19,20,21,28,33$

[88] Yang Bai, Gustavo Burdman, and Christopher T. Hill. Topological Interactions in Warped Extra Dimensions. JHEP, 1002:049, 2010. 13, 15, 17, $25,26,27,28,33$

[89] J. F. Donoghue, E. Golowich, and B. R. Holstein. Dynamics of the Standard Model. Cambridge University Press, 1994. 14

[90] Christopher D. Carone, Joshua Erlich, and Brian Glover. Dynamically warped theory space and collective supersymmetry breaking. JHEP, 0510:042, 2005. 15

[91] Adam Falkowski and Hyung Do Kim. Running of gauge couplings in AdS(5) via deconstruction. JHEP, 0208:052, 2002. 15, 19

[92] Andrey Katz and Yael Shadmi. Gauge theories in AdS(5) and fine-lattice deconstruction. JHEP, 0411:060, 2004. 15, 17

[93] Gustavo Burdman, Pedro Ormonde, and Victor Peralta. Fermion Resonances in Quiver Theories with a pNGB Higgs. JHEP, 11:045, 2014. 16, 22, 55, 60

[94] Leonardo de Lima. New TeV Scale Models for the Gauge and Fermion Mass Hierarquies. PhD thesis, University of São Paulo, 2013. http://www.teses. usp.br/teses/disponiveis/43/43134/tde-29092014-232004/en.php [in Portuguese]. 16, 22, 55, 60

[95] Tony Gherghetta and Alex Pomarol. Bulk fields and supersymmetry in a slice of AdS. Nucl.Phys., B586:141-162, 2000. 18, 28, 32, 33

[96] W. Hahn. Die mechanische Deutung einer geometrischen Differenzgleichung. Zeitschrift für Angewandte Mathematik und Mechanik, 33:270-272, 1953. 18

[97] H. Exton. q-hypergeometric functions and applications. Ellis Hood, 1983. 18 
[98] Lisa Randall, Yael Shadmi, and Neal Weiner. Deconstruction and gauge theories in AdS(5). JHEP, 0301:055, 2003. 19

[99] Hiroyuki Abe, Tatsuo Kobayashi, Nobuhito Maru, and Koichi Yoshioka. Field localization in warped gauge theories. Phys.Rev., D67:045019, 2003. 19

[100] Tony Gherghetta and Alex Pomarol. Bulk fields and supersymmetry in a slice of AdS. Nucl. Phys., B586:141-162, 2000. 19

[101] Yuichiro Nakai. Deconstruction, Holography and Emergent Supersymmetry. JHEP, 03:101, 2015. 20

[102] Yonatan Kahn and Jesse Thaler. Locality in Theory Space. JHEP, 1207:007, 2012. 20

[103] David B. Kaplan and Howard Georgi. SU(2) x U(1) Breaking by Vacuum Misalignment. Phys.Lett., B136:183, 1984. 23, 49

[104] David B. Kaplan, Howard Georgi, and Savas Dimopoulos. Composite Higgs Scalars. Phys.Lett., B136:187, 1984. 23, 49

[105] Roberto Contino, Yasunori Nomura, and Alex Pomarol. Higgs as a holographic pseudoGoldstone boson. Nucl. Phys., B671:148-174, 2003. 24

[106] Yutaka Hosotani and Mitsuru Mabe. Higgs boson mass and electroweak-gravity hierarchy from dynamical gauge-Higgs unification in the warped spacetime. Phys. Lett., B615:257-265, 2005. 24

[107] Adam Falkowski. About the holographic pseudo-Goldstone boson. Phys. Rev., D75:025017, 2007. 24, 60

[108] Naoyuki Haba, Shigeki Matsumoto, Nobuchika Okada, and Toshifumi Yamashita. Effective Potential of Higgs Field in Warped Gauge-Higgs Unification. Prog. Theor. Phys., 120:77-98, 2008. 24

[109] Hsin-Chia Cheng and Ian Low. TeV symmetry and the little hierarchy problem. JHEP, 0309:051, 2003. 24 
[110] Hsin-Chia Cheng and Ian Low. Little hierarchy, little Higgses, and a little symmetry. JHEP, 0408:061, 2004. 24

[111] M. Bona et al. Model-independent constraints on $\Delta \mathrm{F}=2$ operators and the scale of new physics. JHEP, 0803:049, 2008. 39, 40

[112] V. Bertone et al. Kaon Mixing Beyond the SM from Nf=2 tmQCD and model independent constraints from the UTA. JHEP, 1303:089, 2013. 39, 40

[113] LEP Electroweak Working Group. Precision Electroweak Measurements and Constraints on the Standard Model. 2010. [arXiv:1012.2367 [hep-ex]]. 41

[114] Michael E. Peskin and Tatsu Takeuchi. Estimation of oblique electroweak corrections. Phys.Rev., D46:381-409, 1992. 41

[115] Kaustubh Agashe, Antonio Delgado, Michael J. May, and Raman Sundrum. RS1, custodial isospin and precision tests. JHEP, 0308:050, 2003. 44, 61

[116] M. Baak, M. Goebel, J. Haller, A. Hoecker, D. Kennedy, et al. The Electroweak Fit of the Standard Model after the Discovery of a New Boson at the LHC. Eur.Phys.J., C72:2205, 2012. 45

[117] A.D. Martin, W.J. Stirling, R.S. Thorne, and G. Watt. Parton distributions for the LHC. Eur.Phys.J., C63:189-285, 2009.

http://mstwpdf .hepforge.org/. 47

[118] CMS Collaboration. CMS-PAS-EXO-12-059.

http://cds.cern.ch/record/1519066?ln=en. 47, 49

[119] CMS Collaboration. CMS-PAS-EXO-12-023.

http://cds. cern.ch/record/1542405? ln=en. 47

[120] ATLAS Collaboration. ATLAS-CONF-2013-052. https://atlas .web. cern.ch/Atlas/GROUPS/PHYSICS/CONFNOTES/ATLAS-CONF-2013-052/. 47, 49

[121] Ben Lillie, Lisa Randall, and Lian-Tao Wang. The Bulk RS KK-gluon at the LHC. JHEP, 0709:074, 2007. 49 
[122] David H. Lyth. Particle physics models of inflation. Lect. Notes Phys., 738:81-118, 2008. 51

[123] Daniel Baumann. Inflation. In Physics of the large and the small, TASI 09, proceedings of the Theoretical Advanced Study Institute in Elementary Particle Physics, Boulder, Colorado, USA, 1-26 June 2009, pages 523-686, 2011. 51

[124] Nima Arkani-Hamed, Lubos Motl, Alberto Nicolis, and Cumrun Vafa. The String landscape, black holes and gravity as the weakest force. JHEP, 06:060, 2007. 52

[125] Clifford Cheung and Grant N. Remmen. Naturalness and the Weak Gravity Conjecture. Phys. Rev. Lett., 113:051601, 2014. 52, 53

[126] Ben Heidenreich, Matthew Reece, and Tom Rudelius. Weak Gravity Strongly Constrains Large-Field Axion Inflation. JHEP, 12:108, 2015. 52, 53

[127] Ben Heidenreich, Matthew Reece, and Tom Rudelius. Sharpening the Weak Gravity Conjecture with Dimensional Reduction. JHEP, 02:140, 2016. 52, 53

[128] Rick S. Gupta, Zohar Komargodski, Gilad Perez, and Lorenzo Ubaldi. Is the Relaxion an Axion? JHEP, 02:166, 2016. 52

[129] David E. Kaplan and Riccardo Rattazzi. Large field excursions and approximate discrete symmetries from a clockwork axion. Phys. Rev., D93(8):085007, 2016. 52, 55, 60

[130] Kiwoon Choi and Sang Hui Im. Realizing the relaxion from multiple axions and its UV completion with high scale supersymmetry. JHEP, 01:149, 2016. 52,60

[131] Kiwoon Choi and Hyungjin Kim. Aligned natural inflation with modulations. Phys. Lett., B759:520-527, 2016. 52

[132] J. R. Espinosa. Cosmological higgs-axion interplay for a naturally small electroweak scale. http://www.ictp-saifr.org/wp-content/uploads/ 2015/07/11-10-15-Espinosa.pdf, 2015. 52 
[133] Jihn E. Kim, Hans Peter Nilles, and Marco Peloso. Completing natural inflation. JCAP, 0501:005, 2005. 52

[134] Keisuke Harigaya and Masahiro Ibe. Simple realization of inflaton potential on a Riemann surface. Phys. Lett., B738:301-304, 2014. 52

[135] Kiwoon Choi, Hyungjin Kim, and Seokhoon Yun. Natural inflation with multiple sub-Planckian axions. Phys. Rev., D90:023545, 2014. 52

[136] Tetsutaro Higaki and Fuminobu Takahashi. Natural and Multi-Natural Inflation in Axion Landscape. JHEP, 07:074, 2014. 52

[137] Keisuke Harigaya and Masahiro Ibe. Phase Locked Inflation - Effectively Trans-Planckian Natural Inflation. JHEP, 11:147, 2014. 52

[138] Marco Peloso and Caner Unal. Trajectories with suppressed tensor-to-scalar ratio in Aligned Natural Inflation. JCAP, 1506(06):040, 2015. 52

[139] Luis E. Ibanez, Miguel Montero, Angel Uranga, and Irene Valenzuela. Relaxion Monodromy and the Weak Gravity Conjecture. JHEP, 04:020, 2016. 52

[140] Arthur Hebecker, Fabrizio Rompineve, and Alexander Westphal. Axion Monodromy and the Weak Gravity Conjecture. JHEP, 04:157, 2016. 52

[141] Rolf Kappl, Hans Peter Nilles, and Martin Wolfgang Winkler. Modulated Natural Inflation. Phys. Lett., B753:653-659, 2016. 52

[142] D. V. Galtsov and A. A. Ershov. Nonabelian Baldness of Colored Black Holes. Phys. Lett., A138:160-164, 1989. 53

[143] Mikhail S. Volkov and Dmitri V. Gal'tsov. Gravitating nonAbelian solitons and black holes with Yang-Mills fields. Phys. Rept., 319:1-83, 1999. 53

[144] P. Bizon and O. T. Popp. No hair theorem for spherical monopoles and dyons in SU(2) Einstein Yang-Mills theory. Class. Quant. Grav., 9:193-205, 1992. 53 
[145] Burkhard Kleihaus, Jutta Kunz, and Abha Sood. Charged SU(N) Einstein Yang-Mills black holes. Phys. Lett., B418:284-293, 1998. 53

[146] Tom Banks, Matt Johnson, and Assaf Shomer. A Note on Gauge Theories Coupled to Gravity. JHEP, 09:049, 2006. 53

[147] Jason L. Evans, Tony Gherghetta, Natsumi Nagata, and Zachary Thomas. Naturalizing Supersymmetry with a Two-Field Relaxion Mechanism. 2016. [arXiv:1602.04812 [hep-ph]]. 59

[148] Anton de la Fuente, Prashant Saraswat, and Raman Sundrum. Natural Inflation and Quantum Gravity. Phys. Rev. Lett., 114(15):151303, 2015. 62

[149] Subodh P. Patil and Pedro Schwaller. Relaxing the Electroweak Scale: the Role of Broken dS Symmetry. JHEP, 02:077, 2016. 63 